\title{
Relativistic tight-binding approximation method for materials immersed in a uniform magnetic field: Application to crystalline silicon
}

\author{
Katsuhiko Higuchi and Dipendra Bahadur Hamal \\ Graduate School of Advanced Sciences of Matter, Hiroshima University, Higashi-Hiroshima 739-8527, Japan \\ Masahiko Higuchi \\ Department of Physics, Faculty of Science, Shinshu University, Matsumoto 390-8621, Japan \\ (Received 19 September 2014; revised manuscript received 18 December 2014; published 23 February 2015)
}

\begin{abstract}
We present a relativistic tight-binding (TB) approximation method that is applicable to actual crystalline materials immersed in a uniform magnetic field. The magnetic Bloch theorem is used to make the dimensions of the Hamiltonian matrix finite. In addition, by means of the perturbation theory, the magnetic hopping integral that appears in the Hamiltonian matrix is reasonably approximated as the relativistic hopping integral multiplied by the magnetic-field-dependent phase factor. In order to calculate the relativistic hopping integral, the relativistic version of the so-called Slater-Koster table is also given in an explicit form. We apply the present method to crystalline silicon immersed in a uniform magnetic field, and reveal its energy-band structure that is defined in the magnetic first Brillouin zone. It is found that the widths of energy-bands increase with increasing the magnetic field, which indicates the magnetic-field dependence of the appropriateness of the effective mass approximation. The recursive energy spectrum, which is the so-called butterfly diagram, can also be seen in the $\boldsymbol{k}$-space plane perpendicular to the magnetic field.
\end{abstract}

DOI: 10.1103/PhysRevB.91.075122

PACS number(s): 71.15.-m

\section{INTRODUCTION}

Recently, it has been reported that the softening in the elastic constant of the boron-doped silicon is suppressed by an external magnetic field [1]. Such phenomenon, which is observed in low-temperature ultrasonic measurements, attracts much attention because the density of the vacancies in silicon wafers can be estimated from the behavior of the elastic softening [1]. Several authors have studied this phenomenon theoretically by using a kind of model that includes adjustable or phenomenological parameters in the Hamiltonian [2-7]. It is pointed out that the spin-orbit interaction, which is one of the relativistic effects, plays an important role as well as the Zeeman effect [2-7]. In these works, an unusual (negative) value is used for the spin-orbit coupling constant in order to explain the above phenomenon, however, the validity of this approach is open to challenge [7]. If a calculation method of relativistic energy bands for materials immersed in a uniform magnetic field is developed, then it will become a reliable method not only to check the validity of the arguments based on the model Hamiltonian [2-6], but also to investigate the above phenomenon more directly. In other words, as the first step toward clarifying the above phenomenon in a first-principles way, we need a calculation method that can describe the effects of both magnetic field and relativity.

It is generally difficult to incorporate the magnetic field and relativistic effects simultaneously into the calculation method for the electronic structure. Insofar, as the nonrelativistic method goes, the tight-binding (TB) approximation method has been developed and applied to some systems [8-10]. In these nonrelativistic TB method, the Peierls substitution [11] or the Peierls phase [9,13] is utilized to construct the effective Hamiltonian for Bloch electrons in a magnetic field. Calculation methods other than the TB approximation method have also been presented, though relativistic effects are not taken into account in these methods. For example,
Zak proposed a method to use the so-called $k q$ functions as basis functions in calculating the Hamiltonian matrix [14-17]. Actual calculations using $k q$ functions are performed by Obermaier, Schellnhuber, and Rauh [18,19]. In combination of the method using $k q$ functions [14-17] with a canonical transformation [18], they calculated the electronic states for a simple model system in which the magnetic field is applied along the $z$ axis, and the periodic potential is assumed to be separated into an $x, y$-dependent part and a $z$-dependent part [19]. The method by Obermaier et al. $[18,19]$ is also applied to other systems, but all of which are simple model systems $[20,21]$. Thus, unfortunately, the methods by Obermaier et al. $[18,19]$ and by Zak [14-17] are not easy to apply to actual crystalline materials immersed in a magnetic field [22].

Of course, there exist a lot of relativistic methods for the zero magnetic field case. For example, the relativistic linear augmented-plane-wave (RLAPW) method [23-25] has been developed, and it has been successfully applied to $f$-electron materials [25-29]. Thus electronic structure calculation methods that can deal with both relativistic and magnetic field effects simultaneously have not yet been developed so far.

In this paper, we present the relativistic TB approximation method that is applicable to actual materials immersed in a uniform magnetic field. This is hereafter referred to as the magnetic-field-containing relativistic tight-binding approximation method (MFRTB method). Both the magnetic field and relativistic effects are taken into consideration by treating the Dirac equation for an electron that moves in both a uniform magnetic field and the periodic potential of a crystal. The MFRTB method is formulated in the orthodox coordinate representation, as shown in the subsequent sections. It has the following methodological features. (i) The MFRTB method can be applied to actual crystalline materials immersed in a magnetic field. Actually, in this paper, we apply it to a crystalline silicon immersed in a magnetic field. As shown 
in a later section, the electronic structure, including energy gaps, has an explicit dependence on the magnetic field, and a recursive energy spectrum similar to the Hofstadter butterfly diagram [8] is observed. (ii) When performing the above calculations, the hopping integrals of the relativistic TB method need to be used. In this work, we also show that the relativistic hopping integrals can be expressed in the form of a linear combination of several relativistic TB parameters. The explicit expressions for the relativistic hopping integrals are summarized in a tabular form. This table is recognized as the relativistic extension of Slater and Koster's work [30]. As mentioned later, this table is utilized in constructing the MFRTB method. (iii) We can discuss the validity of using Peierls phase $[9,13]$, and show the way to improve it systematically by means of the perturbation theory. The approximation using the Peierls phase $[9,13]$ is regarded as the lowest-order correction when the magnetic field is treated as the perturbation. (iv) We revisit the magnetic Bloch theorem by considering the translation symmetry of the system immersed in the magnetic field. Then, we present a way to determine an expression of the magnetic field that is consistent with the magnetic Bloch theorem. As also shown later, it depends on the structure of the target material.

As mentioned above, the development of the MFRTB method is the first thing to be done for the purpose of revealing the mechanism of the elastic softening and its suppression observed in the boron-doped silicon. In addition to the above application, the MFRTB method may have another interesting application, it could be employed effectively in energy-band calculations for superconductors immersed in a magnetic field [31]. Recently, we have developed the current-density functional theory for superconductors immersed in a magnetic field [31]. In this theory, we have to solve the Kohn-Sham equation of the Bogoliubov-de Gennes type in order to predict the critical temperature and critical magnetic field. This equation may be solved approximately with the aid of the method presented by de Gennes [32], if the wave functions for the normal state are provided through the practical energyband calculation method for materials immersed in a uniform magnetic field. The MFRTB method seems to be useful for such calculations of the normal state.

Furthermore, the MFRTB method can also become a general, first-principles theory to describe physical phenomena observed in a magnetic field. For example, the MFRTB method would give an alternative description of the de Haas-van Alphen (dHvA) and Shubnikov-de Haas effects [33], other than the conventional one [22,34]. Thus the MFRTB method is expected to have a broad utility for the description of various phenomena related to the magnetic field.

The organization of this paper is as follows. In Sec. II, we present a preliminary discussion on the relativistic TB approximation method for the zero magnetic field case. The relativistic version of the Slater-Koster table is given in an explicit form. In Sec. III, the relativistic TB approximation method for the nonzero magnetic field case, i.e., MFRTB method, is presented. The magnetic hopping integrals that appear in the matrix elements of the Hamiltonian are approximated in terms of relativistic hopping integrals for zero magnetic field by means of the perturbation theory. In Sec. IV, we apply the MFRTB method to a two-dimensional square lattice that is immersed in a uniform magnetic field. By comparing the present results with the Hofstadter's ones [8], we discuss the validity and advantages of the MFRTB method. In Sec. V, the energy-band calculation for a crystalline silicon immersed in a uniform magnetic field is performed by means of the MFRTB method. The dependencies of energy spectra both on the wave vector of the magnetic Brillouin zone and on the magnitude of the magnetic field are presented. Finally, some concluding remarks are given in Sec. VI.

\section{RELATIVISTIC TIGHT-BINDING APPROXIMATION METHOD FOR ZERO MAGNETIC FIELD CASE}

In this section, the relativistic TB approximation method for the zero magnetic field case is formulated as a preliminary step toward developing the MFRTB method. The Dirac equation for an electron in a periodic potential is given by [35]

$$
H \Psi_{\alpha, \boldsymbol{k}}(\boldsymbol{r})=\bar{E}_{\alpha, \boldsymbol{k}} \Psi_{\alpha, \boldsymbol{k}}(\boldsymbol{r})
$$

with

$$
H=c \boldsymbol{\alpha} \cdot \boldsymbol{p}+\beta m c^{2}+\sum_{\boldsymbol{R}_{n}} \sum_{i} v_{a_{i}}\left(\boldsymbol{r}-\boldsymbol{R}_{n}-\boldsymbol{d}_{i}\right),
$$

where $c$ and $m$ denote the velocity of light and electron rest mass, respectively, and where $\boldsymbol{\alpha}=\left(\alpha_{x}, \alpha_{y}, \alpha_{z}\right)$ and $\beta$ stand for the usual $4 \times 4$ matrices [35]. In Eq. (2), $v_{a_{i}}\left(\boldsymbol{r}-\boldsymbol{R}_{n}-\boldsymbol{d}_{i}\right)$ is the scalar potential caused by the nucleus of atom $a_{i}$ located at $\boldsymbol{R}_{n}+\boldsymbol{d}_{i}$, where $\boldsymbol{R}_{n}$ and $\boldsymbol{d}_{i}$ denote the translation vector of the lattice and the vector specifying the position of the atom $a_{i}$, respectively. The subscripts $\alpha$ and $\boldsymbol{k}$ of the four-component eigenfunction $\Psi_{\alpha, k}(\boldsymbol{r})$ denote the band index and crystal momentum, respectively. Similar to the nonrelativistic TB approximation method, $\Psi_{\alpha, k}(\boldsymbol{r})$ is expanded by using the Bloch sums of the relativistic atomic orbitals as the basis functions. We have

$$
\Psi_{\alpha, k}(\boldsymbol{r})=\sum_{n l J M} \sum_{i} b_{n l J M, i}^{\alpha, \boldsymbol{k}} B_{n l J M}^{a_{i}, \boldsymbol{k}}(\boldsymbol{r}),
$$

where $b_{n l J M, i}^{\alpha, \boldsymbol{k}}$ is the expansion coefficient, and $B_{n l J M}^{a_{i}, \boldsymbol{k}}(\boldsymbol{r})$ denotes the Bloch sum given by

$$
B_{n l J M}^{a_{i}, \boldsymbol{k}}(\boldsymbol{r})=\frac{1}{\sqrt{N}} \sum_{\boldsymbol{R}_{n}} e^{i \boldsymbol{k} \cdot\left(\boldsymbol{R}_{n}+\boldsymbol{d}_{i}\right)} \varphi_{n l J M}^{a_{i}}\left(\boldsymbol{r}-\boldsymbol{R}_{n}-\boldsymbol{d}_{i}\right)
$$

where $\varphi_{n l J M}^{a_{i}}(\boldsymbol{r})$ is the relativistic atomic orbital of atom $a_{i}$. In Eqs. (3) and (4), $n, l, J$, and $M$ are the principal, azimuthal, total angular momentum, and magnetic quantum numbers, respectively. The number $l$ is related to the parity, which is conserved in the atomic system [35]. The relativistic atomic orbital obeys the following Dirac equation:

$$
\left[c \boldsymbol{\alpha} \cdot \boldsymbol{p}+\beta m c^{2}+v_{a_{i}}(\boldsymbol{r})\right] \varphi_{n l J M}^{a_{i}}(\boldsymbol{r})=\bar{\varepsilon}_{n l J}^{a_{i}} \varphi_{n l J M}^{a_{i}}(\boldsymbol{r}),
$$

where $\bar{\varepsilon}_{n l J}^{a_{i}}$ denotes the atomic spectrum for the zero magnetic field case. It should be noted that $\varphi_{n l J M}^{a_{i}}(\boldsymbol{r})$ is generally written by

$$
\varphi_{n l J M}^{a_{i}}(\boldsymbol{r})=\frac{1}{r}\left[\begin{array}{c}
F_{n l J}^{a_{i}}(r) y_{l, J}^{M}(\theta, \phi) \\
i G_{n l J}^{a_{i}}(r) y_{2 J-l, J}^{M}(\theta, \phi)
\end{array}\right],
$$


where $F_{n l J M}^{a_{i}}(r)$ and $G_{n l J M}^{a_{i}}(r)$ denote the large and small components of the radial part of $\varphi_{n l J M}^{a_{i}}(\boldsymbol{r})$, respectively, and where $y_{l, J}^{M}(\theta, \phi)$ is the spinor spherical harmonics [35].

By neglecting integrals that involve three different centers, we get matrix elements of the Hamiltonian (2) as follows:

$$
\begin{aligned}
& H_{j\left(n^{\prime} l^{\prime} J^{\prime} M^{\prime}\right), i(n l J M)}(\boldsymbol{k}) \\
& =\left\{\bar{\varepsilon}_{n l J}^{a_{i}}+\Delta \bar{\varepsilon}_{n l J M}^{a_{i}, \boldsymbol{d}_{i}}\right\} \delta_{j, i} \delta_{n^{\prime} l^{\prime} J^{\prime} M^{\prime}, n l J M} \\
& +\sum_{\boldsymbol{R}_{n}}\left(1-\delta_{\boldsymbol{R}_{n}, \mathbf{0}} \delta_{j, i}\right) e^{i \boldsymbol{k} \cdot\left(\boldsymbol{R}_{n}+\boldsymbol{d}_{i}-\boldsymbol{d}_{j}\right)} \\
& \quad \times t_{n^{\prime} l^{\prime} J^{\prime} M^{\prime}, n l J M}^{a_{j} a_{i}}\left(\boldsymbol{R}_{n}+\boldsymbol{d}_{i}-\boldsymbol{d}_{j}\right),
\end{aligned}
$$

with

$$
\begin{aligned}
& t_{n^{\prime} l^{\prime} J^{\prime} M^{\prime}, n l J M}^{a_{a} a_{i}}\left(\boldsymbol{R}_{n}+\boldsymbol{d}_{i}-\boldsymbol{d}_{j}\right) \\
& =\int \varphi_{n^{\prime} l^{\prime} J^{\prime} M^{\prime}}^{a_{j}}(\boldsymbol{r})^{\dagger} \frac{v_{a_{j}}(\boldsymbol{r})+v_{a_{i}}\left(\boldsymbol{r}-\boldsymbol{R}_{n}-\boldsymbol{d}_{i}+\boldsymbol{d}_{j}\right)}{2} \\
& \quad \times \varphi_{n l J M}^{a_{i}}\left(\boldsymbol{r}-\boldsymbol{R}_{n}-\boldsymbol{d}_{i}+\boldsymbol{d}_{j}\right) d^{3} r
\end{aligned}
$$

and

$$
\begin{aligned}
\Delta \bar{\varepsilon}_{n l J M}^{a_{i}, \boldsymbol{d}_{i}}= & \int \varphi_{n l J M}^{a_{i}}\left(\boldsymbol{r}-\boldsymbol{d}_{i}\right)^{\dagger}\left\{\sum_{\boldsymbol{R}_{m}} \sum_{k} v_{a_{k}}\left(\boldsymbol{r}-\boldsymbol{R}_{m}-\boldsymbol{d}_{k}\right)\right\} \\
& \times \varphi_{n l J M}^{a_{i}}\left(\boldsymbol{r}-\boldsymbol{d}_{i}\right) d^{3} r
\end{aligned}
$$

In Eqs. (8) and (9), $t_{n^{\prime} l^{\prime} J^{\prime} M^{\prime}, n l J M}^{a_{1} a_{1}}\left(\boldsymbol{R}_{n}+\boldsymbol{d}_{i}-\boldsymbol{d}_{j}\right)$ and $\Delta \bar{\varepsilon}_{n l J M}^{a_{i}, \boldsymbol{d}_{i}}$ denote the relativistic hopping integral and the energy of the crystal field [36], respectively. By using Eq. (6) and the properties of $y_{l, J}^{M}(\theta, \phi)$ [35], it can be proven that $t_{n^{\prime} l^{\prime} J^{\prime} M^{\prime}, n l J M}^{a_{j} a_{i}}\left(\boldsymbol{R}_{n}+\boldsymbol{d}_{i}-\boldsymbol{d}_{j}\right)$ has the following properties:

$$
\begin{aligned}
& \text { (property 1) } \begin{array}{c}
t_{n^{\prime} l^{\prime} J^{\prime} M^{\prime}, n l J M}^{a_{j} a_{i}}\left(\boldsymbol{R}_{n}+\boldsymbol{d}_{i}-\boldsymbol{d}_{j}\right) \\
=t_{n l J M, n^{\prime} l^{\prime} J^{\prime} M^{\prime}}^{a_{i} a_{j}}\left(-\left(\boldsymbol{R}_{n}+\boldsymbol{d}_{i}-\boldsymbol{d}_{j}\right)\right)^{*}
\end{array}
\end{aligned}
$$

(property 2) $\quad t_{n l J M, n^{\prime} l^{\prime} J^{\prime} M^{\prime}}^{a_{i} a_{j}}\left(\boldsymbol{R}_{n}+\boldsymbol{d}_{i}-\boldsymbol{d}_{j}\right)$

$$
=(-1)^{l+l^{\prime}} t_{n^{\prime} l^{\prime} J^{\prime} M^{\prime}, n l J M}^{a_{j} a_{i}}\left(\boldsymbol{R}_{n}+\boldsymbol{d}_{i}-\boldsymbol{d}_{j}\right)^{*} .
$$

Property 1 [Eq. (10)] guarantees the hermicity of the Hamiltonian matrix (7). Property 2 [Eq. (11)] will be used later.

Next, we show that $t_{n^{\prime} l^{\prime} J^{\prime} M^{\prime}, n l J M}^{a_{1} a_{i}}\left(\boldsymbol{R}_{n}+\boldsymbol{d}_{i}-\boldsymbol{d}_{j}\right)$ can be expressed in terms of several relativistic TB parameters, similar to the case of the nonrelativistic hopping integral [30]. With reference to the nonrelativistic case [30,37], the relativistic TB parameter is defined as the relativistic hopping integral between two sites that are placed on the $z$ axis. If atoms $a_{j}$ and $a_{i}$ are placed at the origin and a distance $\left|\boldsymbol{R}_{n}+\boldsymbol{d}_{i}-\boldsymbol{d}_{j}\right|$ away from the origin, respectively, then the relativistic TB parameter is written as $t_{n^{\prime} l^{\prime} J^{\prime} M^{\prime}, n l J M}^{a_{j}}\left(\left|\boldsymbol{R}_{n}+\boldsymbol{d}_{i}-\boldsymbol{d}_{j}\right| \boldsymbol{e}_{z}\right)$, where $\boldsymbol{e}_{z}$ denotes the unit vector in the direction of $z$ axis. This special type of the relativistic hopping integral has the following two properties in addition to properties 1 and 2 [Eqs. (10) and (11)]:

$$
\begin{aligned}
& \text { (property 3) } \begin{array}{c}
t_{n^{\prime} l^{\prime} J^{\prime} M^{\prime}, n l J M}^{a_{j} a_{i}}\left(\left|\boldsymbol{R}_{n}+\boldsymbol{d}_{i}-\boldsymbol{d}_{j}\right| \boldsymbol{e}_{z}\right) \\
=t_{n^{\prime} l^{\prime} J^{\prime} M, n l J M}^{a_{j} a_{i}}
\end{array} \\
& \left(\left|\boldsymbol{R}_{n}+\boldsymbol{d}_{i}-\boldsymbol{d}_{j}\right| \boldsymbol{e}_{z}\right) \delta_{M, M^{\prime}},
\end{aligned}
$$

$$
\begin{gathered}
\text { (property 4) } \quad t_{n^{\prime} l^{\prime} J^{\prime}-M, n l J-M}^{a_{j} a_{i}}\left(\left|\boldsymbol{R}_{n}+\boldsymbol{d}_{i}-\boldsymbol{d}_{j}\right| \boldsymbol{e}_{z}\right) \\
=(-1)^{J+J^{\prime}+l+l^{\prime}+1} t_{n^{\prime} l^{\prime} J^{\prime} M, n l J M}^{a_{j} a_{i}} \\
\times\left(\left|\boldsymbol{R}_{n}+\boldsymbol{d}_{i}-\boldsymbol{d}_{j}\right| \boldsymbol{e}_{z}\right) .
\end{gathered}
$$

Due to properties 3 and 4 [Eqs. (12) and (13)], the relativistic TB parameters can be classified by $n, n^{\prime}, J, J^{\prime}, l, l^{\prime}$, and $|M|$. If we denote the relativistic TB parameter as $K_{d}^{a_{j} a_{i}}\left(n^{\prime} l^{\prime} J^{\prime}, n l J\right)_{|M|}$, then we have

$$
\begin{aligned}
K_{d}^{a_{j} a_{i}}\left(n^{\prime} l^{\prime} J^{\prime}, n l J\right)_{|M|} & \\
= & t_{n^{\prime} l^{\prime} J^{\prime}|M|, n l J|M|}^{a_{j} a_{i}}\left(\left|\boldsymbol{R}_{n}+\boldsymbol{d}_{i}-\boldsymbol{d}_{j}\right| \boldsymbol{e}_{z}\right) \\
= & \int \varphi_{n^{\prime} l^{\prime} J^{\prime}|M|}^{a_{j}}(\boldsymbol{r})^{\dagger} \frac{v_{a_{j}}(\boldsymbol{r})+v_{a_{i}}\left(\boldsymbol{r}-\left|\boldsymbol{R}_{n}+\boldsymbol{d}_{i}-\boldsymbol{d}_{j}\right| \boldsymbol{e}_{z}\right)}{2} \\
& \times \varphi_{n l J|M|}^{a_{i}}\left(\boldsymbol{r}-\left|\boldsymbol{R}_{n}+\boldsymbol{d}_{i}-\boldsymbol{d}_{j}\right| \boldsymbol{e}_{z}\right) d^{3} r
\end{aligned}
$$

where the subscript " $d$ " of $K_{d}^{a_{j} a_{i}}\left(n^{\prime} l^{\prime} J^{\prime}, n l J\right)_{|M|}$ is the parameter that shows the dependence of the relativistic TB parameter on $\left|\boldsymbol{R}_{n}+\boldsymbol{d}_{i}-\boldsymbol{d}_{j}\right|$. Specifically, if $\left|\boldsymbol{R}_{n}+\boldsymbol{d}_{i}-\boldsymbol{d}_{j}\right|$ is equal to the distance between the nearest-neighboring atoms, second-nearest-neighboring atoms, and so on, then $d$ takes the value of $1,2, \ldots$, respectively.

In the case where $l$ takes the values of 0 or 1 , we have ten kinds of relativistic TB parameters, i.e.,

$$
\begin{array}{ll}
K_{d}^{a_{j} a_{i}}\left(n^{\prime} 0 \frac{1}{2}, n 0 \frac{1}{2}\right)_{1 / 2}, & K_{d}^{a_{j} a_{i}}\left(n^{\prime} 0 \frac{1}{2}, n 1 \frac{1}{2}\right)_{1 / 2}, \\
K_{d}^{a_{j} a_{i}}\left(n^{\prime} 0 \frac{1}{2}, n 1 \frac{3}{2}\right)_{1 / 2}, & K_{d}^{a_{j} a_{i}}\left(n^{\prime} 1 \frac{1}{2}, n 0 \frac{1}{2}\right)_{1 / 2}, \\
K_{d}^{a_{j} a_{i}}\left(n^{\prime} 1 \frac{1}{2}, n 1 \frac{1}{2}\right)_{1 / 2}, & K_{d}^{a_{j} a_{i}}\left(n^{\prime} 1 \frac{1}{2}, n 1 \frac{3}{2}\right)_{1 / 2}, \\
K_{d}^{a_{j} a_{i}}\left(n^{\prime} 1 \frac{3}{2}, n 0 \frac{1}{2}\right)_{1 / 2}, & K_{d}^{a_{j} a_{i}}\left(n^{\prime} 1 \frac{3}{2}, n 1 \frac{1}{2}\right)_{1 / 2}, \\
K_{d}^{a_{j} a_{i}}\left(n^{\prime} 1 \frac{3}{2}, n 1 \frac{3}{2}\right)_{1 / 2}, & K_{d}^{a_{j} a_{i}}\left(n^{\prime} 1 \frac{3}{2}, n 1 \frac{3}{2}\right)_{3 / 2} .
\end{array}
$$

In the case of monoatomic crystals such as crystalline silicon, the relativistic TB parameters are, of course, independent of $a_{i}$ and $a_{j}$. Therefore the relativistic TB parameters can be denoted by $K_{d}\left(n^{\prime} l^{\prime} J^{\prime}, n l J\right)_{|M|}$. Due to property 2 [Eq. (11)], $K_{d}\left(n^{\prime} l^{\prime} J^{\prime}, n l J\right)_{|M|}$ and $K_{d}\left(n l J, n^{\prime} l^{\prime} J^{\prime}\right)_{|M|}$ are not independent of each other. Therefore we have seven kinds of relativistic TB parameters in this case, i.e.,

$$
\begin{array}{ll}
K_{d}\left(n^{\prime} 0 \frac{1}{2}, n 0 \frac{1}{2}\right)_{1 / 2}, & K_{d}\left(n^{\prime} 0 \frac{1}{2}, n 1 \frac{1}{2}\right)_{1 / 2}, \\
K_{d}\left(n^{\prime} 0 \frac{1}{2}, n 1 \frac{3}{2}\right)_{1 / 2}, & K_{d}\left(n^{\prime} 1 \frac{1}{2}, n 1 \frac{1}{2}\right)_{1 / 2}, \\
K_{d}\left(n^{\prime} 1 \frac{1}{2}, n 1 \frac{3}{2}\right)_{1 / 2}, & K_{d}\left(n^{\prime} 1 \frac{3}{2}, n 1 \frac{3}{2}\right)_{1 / 2}, \\
K_{d}\left(n^{\prime} 1 \frac{3}{2}, n 1 \frac{3}{2}\right)_{3 / 2} . &
\end{array}
$$


In order to express $K_{d}^{a_{j} a_{i}}\left(n^{\prime} l^{\prime} J^{\prime}, n l J\right)_{|M|}$ by using the large and small components of the relativistic atomic orbitals, we introduce the following notations:

$$
\begin{aligned}
& \left(\overline{l^{\prime}} \bar{l} \bar{M}\right)_{\left(n^{\prime} l^{\prime} J^{\prime}, n l J\right)}^{L, d, a_{j} a_{i}}=\int \frac{1}{r r_{i j n}^{z}} F_{n^{\prime} l^{\prime} J^{\prime}}^{a_{j}}(r)^{*} Y_{\bar{l}^{\prime}, \bar{M}}^{*}(\theta, \phi) \frac{v_{a_{j}}(\boldsymbol{r})+v_{a_{i}}\left(\boldsymbol{r}-\left|\boldsymbol{R}_{n}+\boldsymbol{d}_{i}-\boldsymbol{d}_{j}\right| \boldsymbol{e}_{z}\right)}{2} F_{n l J}^{a_{i}}\left(r_{i j n}^{z}\right) Y_{\bar{l}, \bar{M}}\left(\theta_{i j n}^{z}, \phi\right) d^{3} r, \\
& \left(\overline{l^{\prime}} \bar{l} \bar{M}\right)_{\substack{\left.n^{\prime} l^{\prime} J^{\prime}, n l J\right) \\
S, d, a_{j} a_{i}}}^{z}=\int \frac{1}{r r_{i j n}^{z}} G_{n^{\prime} l^{\prime} J^{\prime}}^{a_{j}}(r)^{*} Y_{\bar{l}^{\prime}, \bar{M}}^{*}(\theta, \phi) \frac{v_{a_{j}}(\boldsymbol{r})+v_{a_{i}}\left(\boldsymbol{r}-\left|\boldsymbol{R}_{n}+\boldsymbol{d}_{i}-\boldsymbol{d}_{j}\right| \boldsymbol{e}_{z}\right)}{2} G_{n l J}^{a_{i}}\left(r_{i j n}^{z}\right) Y_{\bar{l}, \bar{M}}\left(\theta_{i j n}^{z}, \phi\right) d^{3} r,
\end{aligned}
$$

where $Y_{\bar{l}^{\prime}, \bar{m}}(\theta, \phi)$ are the spherical harmonics, and where the arguments $\left(r_{i j n}^{z}, \theta_{i j n}^{z}, \phi\right)$ stand for the polar coordinates of $\boldsymbol{r}-$ $\left|\boldsymbol{R}_{n}+\boldsymbol{d}_{i}-\boldsymbol{d}_{j}\right| \boldsymbol{e}_{z}$. The superscript " $L$ " (or " $S$ ") indicates that the integral comes from the large (or small) component of the relativistic atomic orbital. The superscript " $d$ " has the same meaning as that mentioned below Eq. (14). By convention, label $\bar{l}^{\prime}($ or $\bar{l})$ is denoted by $s, p, d, \ldots$ for $\bar{l}^{\prime}$ ( or $\left.\bar{l}\right)=0,1,2, \ldots$, respectively, and the label $\bar{M}$ is denoted by $\sigma, \pi, \delta, \ldots$, for $\bar{M}=0, \pm 1, \pm 2, \ldots$, respectively. For example, we have

$$
\begin{gathered}
(s p \sigma)_{\left(n^{\prime} 0 \frac{1}{2}, n 0 \frac{1}{2}\right)}^{L, d, a_{j} a_{i}}=\int \frac{1}{r r_{i j n}^{z}} F_{n^{\prime} 0 \frac{1}{2}}^{a_{j}}(r)^{*} Y_{0,0}^{*}(\theta, \phi) \frac{v_{a_{j}}(\boldsymbol{r})+v_{a_{i}}\left(\boldsymbol{r}-\left|\boldsymbol{R}_{n}+\boldsymbol{d}_{i}-\boldsymbol{d}_{j}\right| \boldsymbol{e}_{z}\right)}{2} F_{n 0 \frac{1}{2}}^{a_{i}}\left(r_{i j n}^{z}\right) Y_{1,0}\left(\theta_{i j n}^{z}, \phi\right) d^{3} r, \\
(p p \pi)_{\left(n^{\prime} 1 \frac{1}{2}, n 1 \frac{1}{2}\right)}^{S, d, a_{j} a_{i}}=\int \frac{1}{r r_{i j n}^{z}} G_{n^{\prime} 1 \frac{1}{2}}^{a_{j}}(r)^{*} Y_{1,1}^{*}(\theta, \phi) \frac{v_{a_{j}}(\boldsymbol{r})+v_{a_{i}}\left(\boldsymbol{r}-\left|\boldsymbol{R}_{n}+\boldsymbol{d}_{i}-\boldsymbol{d}_{j}\right| \boldsymbol{e}_{z}\right)}{2} G_{n^{\prime} 1 \frac{1}{2}}^{a_{i}}\left(r_{i j n}^{z}\right) Y_{1,1}\left(\theta_{i j n}^{z}, \phi\right) d^{3} r .
\end{gathered}
$$

Using this notation, the ten kinds of $K_{d}^{a_{j} a_{i}}\left(n^{\prime} l^{\prime} J^{\prime}, n l J\right)_{|M|}$ 's shown above are expressed as

$$
\begin{aligned}
& K_{d}^{a_{j} a_{i}}\left(n^{\prime} 0 \frac{1}{2}, n 0 \frac{1}{2}\right)_{1 / 2}=(s s \sigma)_{\left(n^{\prime} 0 \frac{1}{2}, n 0 \frac{1}{2}\right)}^{L, d, a_{j} a_{i}}+\frac{1}{3}(p p \sigma)_{\left(n^{\prime} 0 \frac{1}{2}, n 0 \frac{1}{2}\right)}^{S, d, a_{j} a_{i}}+\frac{2}{3}(p p \pi)_{\left(n^{\prime} 0 \frac{1}{2}, n 0 \frac{1}{2}\right)}^{S, d, a_{j} a_{i}}, \\
& K_{d}^{a_{j} a_{i}}\left(n^{\prime} 0 \frac{1}{2}, n 1 \frac{1}{2}\right)_{1 / 2}=-\frac{1}{\sqrt{3}}(\operatorname{sp} \sigma)_{\left(n^{\prime} 0 \frac{1}{2}, n 0 \frac{1}{2}\right)}^{L, d, a_{j} a_{i}}-\frac{1}{\sqrt{3}}(p s \sigma)_{\left(n^{\prime} 0 \frac{1}{2}, n 1 \frac{1}{2}\right)}^{S, d, a_{j} a_{i}}, \\
& K_{d}^{a_{j} a_{i}}\left(n^{\prime} 0 \frac{1}{2}, n 1 \frac{3}{2}\right)_{1 / 2}=\sqrt{\frac{2}{3}}(s p \sigma)_{\left(n^{\prime} 0 \frac{1}{2}, n 0 \frac{3}{2}\right)}^{L, d, a_{j} a_{i}}+\sqrt{\frac{2}{15}}(p d \sigma)_{\left(n^{\prime} 0 \frac{1}{2}, n 0 \frac{3}{2}\right)}^{S, d a_{j} a_{i}}+\sqrt{\frac{2}{5}}(p d \pi)_{\left(n^{\prime} 0 \frac{1}{2}, n 0 \frac{3}{2}\right)}^{S, d, a_{j} a_{i}}, \\
& K_{d}^{a_{j} a_{i}}\left(n^{\prime} 1 \frac{1}{2}, n 0 \frac{1}{2}\right)_{1 / 2}=-\frac{1}{\sqrt{3}}(p s \sigma)_{\left(n^{\prime} 1 \frac{1}{2}, n 0 \frac{1}{2}\right)}^{L, d, a_{j} a_{i}}-\frac{1}{\sqrt{3}}(s p \sigma)_{\left(n^{\prime} 1 \frac{1}{2}, n 0 \frac{1}{2}\right)}^{S, d, a_{j} a_{i}}, \\
& K_{d}^{a_{j} a_{i}}\left(n^{\prime} 1 \frac{1}{2}, n 1 \frac{1}{2}\right)_{1 / 2}=\frac{1}{3}(p p \sigma)_{\left(n^{\prime} 1 \frac{1}{2}, n 1 \frac{1}{2}\right)}^{L, d, a_{j} a_{i}}+\frac{2}{3}(p p \pi)_{\left(n^{\prime} 1 \frac{1}{2}, n 1 \frac{1}{2}\right)}^{L, d, a_{j} a_{i}}+(s s \sigma)_{\left(n^{\prime} 1 \frac{1}{2}, n 1 \frac{1}{2}\right)}^{S, d, a_{j} a_{i}}, \\
& K_{d}^{a_{j} a_{i}}\left(n^{\prime} 1 \frac{1}{2}, n 1 \frac{3}{2}\right)_{1 / 2}=-\frac{\sqrt{2}}{3}(p p \sigma)_{\left(n^{\prime} 1 \frac{1}{2}, n 1 \frac{3}{2}\right)}^{L, d, a_{j} a_{i}}+\frac{\sqrt{2}}{3}(p p \pi)_{\left(n^{\prime} 1 \frac{1}{2}, n 1 \frac{3}{2}\right)}^{L, d a_{j} a_{i}}-\sqrt{\frac{2}{5}}(s d \sigma)_{\left(n^{\prime} 1 \frac{1}{2}, n 1 \frac{3}{2}\right)}^{S, d, a_{j} a_{i}}, \\
& K_{d}^{a_{j} a_{i}}\left(n^{\prime} 1 \frac{3}{2}, n 0 \frac{1}{2}\right)_{1 / 2}=\sqrt{\frac{2}{3}}(p s \sigma)_{\left(n^{\prime} 1 \frac{3}{2}, n 0 \frac{1}{2}\right)}^{L, d, a_{j} a_{i}}+\sqrt{\frac{2}{15}}(d p \sigma)_{\left(n^{\prime} 1 \frac{3}{2}, n 0 \frac{1}{2}\right)}^{S, d, a_{j} a_{i}}+\sqrt{\frac{2}{5}}(d p \pi)_{\left(n^{\prime} 1 \frac{3}{2}, n 0 \frac{1}{2}\right)}^{S, d, a_{j} a_{i}}, \\
& K_{d}^{a_{j} a_{i}}\left(n^{\prime} 1 \frac{3}{2}, n 1 \frac{1}{2}\right)_{1 / 2}=-\frac{\sqrt{2}}{3}(p p \sigma)_{\left(n^{\prime} 1 \frac{3}{2}, n 1 \frac{1}{2}\right)}^{L, d, a_{j} a_{i}}+\frac{\sqrt{2}}{3}(p p \pi)_{\left(n^{\prime} 1 \frac{3}{2}, n 1 \frac{1}{2}\right)}^{L, d a_{j} a_{i}}-\sqrt{\frac{2}{5}}(d s \sigma)_{\left(n^{\prime} 1 \frac{3}{2}, n 1 \frac{1}{2}\right)}^{S, d, a_{j} a_{i}}, \\
& K_{d}^{a_{j} a_{i}}\left(n^{\prime} 1 \frac{3}{2}, n 1 \frac{3}{2}\right)_{1 / 2}=\frac{2}{3}(p p \sigma)_{\left(n^{\prime} 1 \frac{3}{2}, n 1 \frac{3}{2}\right)}^{L, d, a_{j} a_{i}}+\frac{1}{3}(p p \pi)_{\left(n^{\prime} 1 \frac{3}{2}, n 1 \frac{3}{2}\right)}^{L, d, a_{j} a_{i}}+\frac{2}{5}(d d \sigma)_{\left(n^{\prime} 1 \frac{3}{2}, n 1 \frac{3}{2}\right)}^{S, d, a_{j} a_{i}}+\frac{3}{5}(d d \pi)_{\left(n^{\prime} 1 \frac{3}{2}, n 1 \frac{3}{2}\right)}^{S, d, a_{j} a_{i}}, \\
& K_{d}^{a_{j} a_{i}}\left(n^{\prime} 1 \frac{3}{2}, n 1 \frac{3}{2}\right)_{3 / 2}=(p p \pi)_{\left(n^{\prime} 1 \frac{3}{2}, n 1 \frac{3}{2}\right)}^{L, d, a_{j} a_{i}}+\frac{1}{5}(d d \pi)_{\left(n^{\prime} 1 \frac{3}{2}, n 1 \frac{3}{2}\right)}^{S, d, a_{j} a_{i}}+\frac{4}{5}(d d \delta)_{\left(n^{\prime} 1 \frac{3}{2}, n 1 \frac{3}{2}\right)}^{S, d, a_{j} a_{i}} .
\end{aligned}
$$

It is clear from the above that $K_{d}^{a_{j} a_{i}}\left(n^{\prime} l^{\prime} J^{\prime}, n l J\right)_{|M|}$ contains contributions that come from not only the large component but also the small component of the relativistic atomic orbital.

In the nonrelativistic TB approximation method, Slater and Koster provided a useful table, in which nonrelativistic hopping integrals are expressed in terms of several TB parameters [30]. The table is called "Slater-Koster table" [30]. With the aid of the Slater-Koster table, the relativistic version of the Slater-Koster table can be derived. For instance, using Eqs. (6) and (8), the 
explicit form of $t_{n^{\prime} 0 \frac{1}{2} \frac{1}{2}, n 1 \frac{1}{2} \frac{1}{2}}^{a_{j} a_{i}}\left(\boldsymbol{R}_{n}+\boldsymbol{d}_{i}-\boldsymbol{d}_{j}\right)$ is given by

$$
\begin{aligned}
t_{n^{\prime} 0 \frac{1}{2} \frac{1}{2}, n 1 \frac{1}{2} \frac{1}{2}}^{a_{a} a_{i}}\left(\boldsymbol{R}_{n}+\boldsymbol{d}_{i}-\boldsymbol{d}_{j}\right)= & -\frac{1}{\sqrt{3}} \int \frac{1}{r r_{i j n}} F_{n^{\prime} 0 \frac{1}{2}}^{a_{j}}(r)^{*} Y_{0,0}^{*}(\theta, \phi) \frac{v_{a_{j}}(\boldsymbol{r})+v_{a_{i}}\left(\boldsymbol{r}-\boldsymbol{R}_{n}-\boldsymbol{d}_{i}+\boldsymbol{d}_{j}\right)}{2} F_{n 1 \frac{1}{2}}^{a_{i}}\left(r_{i j n}\right) Y_{1,0}\left(\theta_{i j n}, \phi_{i j n}\right) d^{3} r \\
& -\frac{1}{\sqrt{3}} \int \frac{1}{r r_{i j n}} G_{n^{\prime} 0 \frac{1}{2}}^{a_{j}}(r)^{*} Y_{1,0}^{*}(\theta, \phi) \frac{v_{a_{j}}(\boldsymbol{r})+v_{a_{i}}\left(\boldsymbol{r}-\boldsymbol{R}_{n}-\boldsymbol{d}_{i}+\boldsymbol{d}_{j}\right)}{2} G_{n 1 \frac{1}{2}}^{a_{i}}\left(r_{i j n}\right) Y_{0,0}\left(\theta_{i j n}, \phi_{i j n}\right) d^{3} r \\
= & -\frac{1}{\sqrt{3}} \int \frac{1}{r r_{i j n}} F_{n^{\prime} 0 \frac{1}{2}}^{a_{j}}(r)^{*} C_{s}^{*}(\theta, \phi) \frac{v_{a_{j}}(\boldsymbol{r})+v_{a_{i}}\left(\boldsymbol{r}-\boldsymbol{R}_{n}-\boldsymbol{d}_{i}+\boldsymbol{d}_{j}\right)}{2} F_{n 1 \frac{1}{2}}^{a_{i}}\left(r_{i j n}\right) C_{z}\left(\theta_{i j n}, \phi_{i j n}\right) d^{3} r \\
& -\frac{1}{\sqrt{3}} \int \frac{1}{r r_{i j n}} G_{n^{\prime} 0 \frac{1}{2}}^{a_{j}}(r)^{*} C_{z}^{*}(\theta, \phi) \frac{v_{a_{j}}(\boldsymbol{r})+v_{a_{i}}\left(\boldsymbol{r}-\boldsymbol{R}_{n}-\boldsymbol{d}_{i}+\boldsymbol{d}_{j}\right)}{2} G_{n 1 \frac{1}{2}}^{a_{i}}\left(r_{i j n}\right) C_{s}\left(\theta_{i j n}, \phi_{i j n}\right) d^{3} r,
\end{aligned}
$$

where the arguments $\left(r_{i j n}, \theta_{i j n}, \phi_{i j n}\right)$ stand for the polar coordinates of $\boldsymbol{r}-\boldsymbol{R}_{n}-\boldsymbol{d}_{i}+\boldsymbol{d}_{j}$, and where $C_{s}(\theta, \phi)$ and $C_{z}(\theta, \phi)$ denote the cubic harmonics (real spherical harmonics) [37]. By applying the results of the Slater-Koster table [30] to each term of the right-hand side of Eq. (20), we have

$$
\begin{aligned}
& t_{n^{\prime} 0 \frac{1}{2} \frac{1}{2}, n 1 \frac{1}{2} \frac{1}{2}}^{a_{j} a_{i}}\left(\boldsymbol{R}_{n}+\boldsymbol{d}_{i}-\boldsymbol{d}_{j}\right) \\
& =-\frac{1}{\sqrt{3}} q_{z}(\operatorname{sp} \sigma)_{\left(n^{\prime} 0 \frac{1}{2}, n 1 \frac{1}{2}\right)}^{L, d, a_{j} a_{i}}-\frac{1}{\sqrt{3}} q_{z}(p s \sigma)_{\left(n^{\prime} 0 \frac{1}{2}, n 1 \frac{1}{2}\right)}^{S, d, a_{j} a_{i}} \\
& \quad=q_{z} K_{d}^{a_{j} a_{i}}\left(n^{\prime} 0 \frac{1}{2}, n 1 \frac{1}{2}\right)_{1 / 2},
\end{aligned}
$$

where the direction cosine of $\boldsymbol{R}_{n}+\boldsymbol{d}_{i}-\mathbf{d}_{j}$ is denoted as $\left(q_{x}, q_{y}, q_{z}\right)$. From the first line to the second line of the right-hand side, Eq. (19) is utilized. In a similar way to the above example, we derive explicit expressions for all other relativistic hopping integrals $t_{n^{\prime} l^{\prime} J^{\prime} M^{\prime}, n l J M}^{a_{1} a_{i}}\left(\boldsymbol{R}_{n}+\boldsymbol{d}_{i}-\boldsymbol{d}_{j}\right)$. The results are summarized in Table I. These explicit expressions for $t_{n^{\prime} l^{\prime} J^{\prime} M^{\prime}, n l J M}^{a_{a} a_{i}}\left(\boldsymbol{R}_{n}+\boldsymbol{d}_{i}-\boldsymbol{d}_{j}\right)$ come in really useful, because we can readily calculate $t_{n^{\prime} l^{\prime} J^{\prime} M^{\prime}, n l J M}^{a_{i} a_{j}}\left(\boldsymbol{R}_{n}+\boldsymbol{d}_{i}-\boldsymbol{d}_{j}\right)$ through values of $K_{d}^{a_{j} a_{i}}\left(n^{\prime} l^{\prime} J^{\prime}, n l J\right)_{|M|}$ and the direction cosines of $\boldsymbol{R}_{n}+\boldsymbol{d}_{i}-\boldsymbol{d}_{j}$ with the aid of Table I. Table I can be recognized as the relativistic version of the Slater-Koster table [30], and is referred to as the relativistic Slater-Koster table hereafter.

It should be noted that the expressions for $t_{n^{\prime} l^{\prime} J^{\prime} M^{\prime}, n l J M}^{a_{j} a_{i}}\left(\boldsymbol{R}_{n}+\boldsymbol{d}_{i}-\boldsymbol{d}_{j}\right)$ and $t_{n^{\prime} l J M, n l^{\prime} J^{\prime} M^{\prime}}^{a_{j} a_{i}}\left(\boldsymbol{R}_{n}+\boldsymbol{d}_{i}-\boldsymbol{d}_{j}\right)$ are related to each other. We shall briefly explain this relation. As seen in Table I, $t_{n^{\prime} l^{\prime} J^{\prime} M^{\prime}, n l J M}^{a_{a} a_{i}}\left(\boldsymbol{R}_{n}+\boldsymbol{d}_{i}-\boldsymbol{d}_{j}\right)$ is generally expressed in the form of the following linear combination of $K_{d}^{a_{j} a_{i}}\left(n^{\prime} l^{\prime} J^{\prime}, n l J\right)_{|M|}$ :

$$
\begin{aligned}
& t_{n^{\prime} l^{\prime} J^{\prime} M^{\prime}, n l J M}^{a_{a} a_{i}}\left(\boldsymbol{R}_{n}+\boldsymbol{d}_{i}-\boldsymbol{d}_{j}\right) \\
& \quad=\sum_{|M|} f\left(q_{x}, q_{y}, q_{z},|M|, l^{\prime} J^{\prime}, l J\right) K_{d}^{a_{j} a_{i}}\left(n^{\prime} l^{\prime} J^{\prime}, n l J\right)_{|M|},
\end{aligned}
$$

where $f\left(q_{x}, q_{y}, q_{z},|M|, l^{\prime} J^{\prime}, l J\right)$ represents the coefficient, and its specific form can be found in Table I. It is shown by using property $2[\mathrm{Eq} .(11)]$ and Eq. (22) that $t_{n^{\prime} l J M, n l^{\prime} J^{\prime} M^{\prime}}^{a_{i} a_{i}}\left(\boldsymbol{R}_{n}+\right.$ $\left.\boldsymbol{d}_{i}-\boldsymbol{d}_{j}\right)$, the subscripts of which, except the principal quantum numbers, are exchanged compared to $t_{n^{\prime} l^{\prime} J^{\prime} M^{\prime}, n l J M}^{a a_{i}}\left(\boldsymbol{R}_{n}+\boldsymbol{d}_{i}-\right.$ $\boldsymbol{d}_{j}$ ), can be expressed by using the coefficients that appear in
Eq. (22), i.e.,

$$
\begin{aligned}
& t_{n^{\prime} l J M, n l^{\prime} J^{\prime} M^{\prime}}^{a_{j} a_{i}}\left(\boldsymbol{R}_{n}+\boldsymbol{d}_{i}-\boldsymbol{d}_{j}\right) \\
& \quad=\sum_{|M|} f\left(q_{x}, q_{y}, q_{z},|M|, l^{\prime} J^{\prime}, l J\right)^{*} K_{d}^{a_{j} a_{i}}\left(n^{\prime} l J, n l^{\prime} J^{\prime}\right)|M| \cdot
\end{aligned}
$$

Equation (23) means that if the explicit expression for $t_{n l^{\prime} J^{\prime} M^{\prime}, n^{\prime} l J M}^{a_{j} a_{i}}\left(\boldsymbol{R}_{n}+\boldsymbol{d}_{i}-\boldsymbol{d}_{j}\right)$ is obtained like Eq. (22), then we can easily get the expression for $t_{n^{\prime} l J M, n l^{\prime} J^{\prime} M^{\prime}}^{a_{i} a_{i}}\left(\boldsymbol{R}_{n}+\right.$ $\left.\boldsymbol{d}_{i}-\boldsymbol{d}_{j}\right)$ by replacing $f\left(q_{x}, q_{y}, q_{z},|M|, l^{\prime} \boldsymbol{J}^{\prime}, l \boldsymbol{J}\right)$ and $K_{d}^{a_{j} a_{i}}\left(n^{\prime} l^{\prime} J^{\prime}, n l J\right)_{|M|}$ with $f\left(q_{x}, q_{y}, q_{z},|M|, l^{\prime} J^{\prime}, l J\right)^{*}$ and $K_{d}^{a_{j} a_{i}}\left(n^{\prime} l J, n l^{\prime} J^{\prime}\right)_{|M|}$, respectively. This relation is regarded as one of the properties of the relativistic hopping integral. Thus we have

(Property 5): An expression for $t_{n^{\prime} l J M, n l^{\prime} J^{\prime} M^{\prime}}^{a_{i} a_{i}}\left(\boldsymbol{R}_{n}+\boldsymbol{d}_{i}-\boldsymbol{d}_{j}\right)$ can be derived from that for $t_{n^{\prime} l^{\prime} J^{\prime} M^{\prime}, n l J M}^{a_{j} a_{i}}\left(\boldsymbol{R}_{n}+\boldsymbol{d}_{i}-\boldsymbol{d}_{j}\right)$.

Property 5 is actually used in deriving the expressions for some of the relativistic hopping integrals in Table I.

At the end of this section, we briefly explain how to determine the values of relativistic TB parameters $K_{d}^{a_{j} a_{i}}\left(n^{\prime} l^{\prime} J^{\prime}, n l J\right)_{|M|}$. In this paper, the relativistic TB parameters between the nearest-neighbor atoms are determined for the crystalline silicon, bearing in mind that the MFRTB method will be actually applied to a crystalline silicon immersed in a magnetic field (Sec. V). At first, Eq. (7) is applied to a crystalline silicon with taking into consideration the hopping integrals between the outermost shells of the nearest-neighbor atoms. Namely, the following eight kinds of relativistic atomic orbitals for each silicon atom are taken into consideration:

$$
\begin{array}{r}
(n, l, J, M)=\left(3,0, \frac{1}{2}, \pm \frac{1}{2}\right),\left(3,1, \frac{1}{2}, \pm \frac{1}{2}\right), \\
\left(3,1, \frac{3}{2}, \pm \frac{1}{2}\right),\left(3,1, \frac{3}{2}, \pm \frac{3}{2}\right) .
\end{array}
$$

The Hamiltonian matrix thus obtained has the dimension of $16 \times 16$ because there are two silicon atoms in the unit cell. Next, the relativistic TB parameters are determined by requiring that the eigenvalues of the Hamiltonian matrix coincide with the reference data as well as possible. As the reference data, we utilize results calculated by the fully RLAPW method [25,28,29]. As for $\bar{\varepsilon}_{n l J}^{a_{i}}+\Delta \bar{\varepsilon}_{n l J M}^{a_{i}, \boldsymbol{d}_{i}}$ that is included in Eq. (7), since the energy of the crystal field is 
TABLE I. Relativistic version of the Slater-Koster table. In the table, $q_{x}, q_{y}$, and $q_{z}$ denote the direction cosines of $\boldsymbol{R}_{n}+\boldsymbol{d}_{i}-\boldsymbol{d}_{j}$.

\begin{tabular}{|c|c|c|}
\hline$\left(n^{\prime}, l^{\prime}, J^{\prime}, M^{\prime}\right)$ & $(n, l, J, M)$ & Hopping integrals $t_{n^{\prime} l^{\prime} J^{\prime} M^{\prime}, n l J M}^{a_{j} a_{i}}\left(\boldsymbol{R}_{n}+\boldsymbol{d}_{i}-\boldsymbol{d}_{j}\right)$ \\
\hline$\left(n^{\prime}, 0, \frac{1}{2}, \frac{1}{2}\right)$ & $\left(n, 0, \frac{1}{2}, \frac{1}{2}\right)$ & $K_{d}^{a_{j} a_{i}}\left(n^{\prime} 0 \frac{1}{2}, n 0 \frac{1}{2}\right)_{1 / 2}$ \\
\hline$\left(n^{\prime}, 0, \frac{1}{2}, \frac{1}{2}\right)$ & $\left(n, 0, \frac{1}{2},-\frac{1}{2}\right)$ & $\begin{array}{ccc}2 & 2 / 1 / 2 \\
0 & 0\end{array}$ \\
\hline$\left(n^{\prime}, 0, \frac{1}{2}, \frac{1}{2}\right)$ & $\left(n, 1, \frac{1}{2}, \frac{1}{2}\right)$ & $q_{z} K_{d}^{a_{j} a_{i}}\left(n^{\prime} 0 \frac{1}{2}, n 1 \frac{1}{2}\right)_{1 / 2}$ \\
\hline$\left(n^{\prime}, 0, \frac{1}{2}, \frac{1}{2}\right)$ & $\left(n, 1, \frac{1}{2},-\frac{1}{2}\right)$ & $\left(q_{x}-i q_{y}\right) K_{d}^{a_{j} a_{i}}\left(n^{\prime} 0 \frac{1}{2}, n 1 \frac{1}{2}\right)_{1 / 2}$ \\
\hline$\left(n^{\prime}, 0, \frac{1}{2}, \frac{1}{2}\right)$ & $\left(n, 1, \frac{3}{2}, \frac{3}{2}\right)$ & $-\frac{\sqrt{3}}{2}\left(q_{x}+i q_{y}\right) K_{d}^{a_{j} a_{i}}\left(n^{\prime} 0 \frac{1}{2}, n 1 \frac{3}{2}\right)_{1 / 2}$ \\
\hline$\left(n^{\prime}, 0, \frac{1}{2}, \frac{1}{2}\right)$ & $\left(n, 1, \frac{3}{2}, \frac{1}{2}\right)$ & $q_{z} K_{d}^{a_{j} a_{i}}\left(n^{\prime} 0 \frac{1}{2}, n 1 \frac{3}{2}\right)_{1 / 2}$ \\
\hline$\left(n^{\prime}, 0, \frac{1}{2}, \frac{1}{2}\right)$ & $\left(n, 1, \frac{3}{2},-\frac{1}{2}\right)$ & $\frac{q_{x}-i q_{y}}{2} K_{d}^{a_{j} a_{i}}\left(n^{\prime} 0 \frac{1}{2}, n 1 \frac{3}{2}\right)_{1 / 2}$ \\
\hline$\left(n^{\prime}, 0, \frac{1}{2}, \frac{1}{2}\right)$ & $\left(n, 1, \frac{3}{2},-\frac{3}{2}\right)$ & 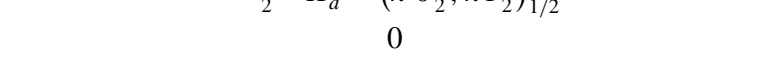 \\
\hline$\left(n^{\prime}, 0, \frac{1}{2},-\frac{1}{2}\right)$ & $\left(n, 0, \frac{1}{2}, \frac{1}{2}\right)$ & 0 \\
\hline$\left(n^{\prime}, 0, \frac{1}{2},-\frac{1}{2}\right)$ & $\left(n, 0, \frac{1}{2},-\frac{1}{2}\right)$ & $K_{d}^{a_{j} a_{i}}\left(n^{\prime} 0 \frac{1}{2}, n 0 \frac{1}{2}\right)_{1 / 2}$ \\
\hline$\left(n^{\prime}, 0, \frac{1}{2},-\frac{1}{2}\right)$ & $\left(n, 1, \frac{1}{2}, \frac{1}{2}\right)$ & $\left(q_{x}+i q_{y}\right) K_{d}^{a_{j} a_{i}^{2}}\left(n^{\prime} 0 \frac{1}{2}, n 1 \frac{1}{2}\right)_{1 / 2}$ \\
\hline$\left(n^{\prime}, 0, \frac{1}{2},-\frac{1}{2}\right)$ & $\left(n, 1, \frac{1}{2},-\frac{1}{2}\right)$ & $-q_{z} K_{d}^{a_{j} a_{i}}\left(n^{\prime} 0 \frac{1}{2}, n 1 \frac{1}{2}\right)_{1 / 2}$ \\
\hline$\left(n^{\prime}, 0, \frac{1}{2},-\frac{1}{2}\right)$ & $\left(n, 1, \frac{3}{2}, \frac{3}{2}\right)$ & 0 \\
\hline$\left(n^{\prime}, 0, \frac{1}{2},-\frac{1}{2}\right)$ & $\left(n, 1, \frac{3}{2}, \frac{1}{2}\right)$ & $-\frac{q_{x}+i q_{y}}{2} K_{d}^{a_{j} a_{i}}\left(n^{\prime} 0 \frac{1}{2}, n 1 \frac{3}{2}\right)_{1 / 2}$ \\
\hline$\left(n^{\prime}, 0, \frac{1}{2},-\frac{1}{2}\right)$ & $\left(n, 1, \frac{3}{2},-\frac{1}{2}\right)$ & $q_{z} K_{d}^{a_{j} a_{i}}\left(n^{\prime} 0 \frac{1}{2}, n 1 \frac{3}{2}\right)_{1 / 2}$ \\
\hline$\left(n^{\prime}, 0, \frac{1}{2},-\frac{1}{2}\right)$ & $\left(n, 1, \frac{3}{2},-\frac{3}{2}\right)$ & $\frac{\sqrt{3}}{2}\left(q_{x}-i q_{y}\right) K_{d}^{a_{j} a_{i}}\left(n^{\prime} 0 \frac{1}{2}, n 1 \frac{3}{2}\right)_{1 / 2}$ \\
\hline$\left(n^{\prime}, 1, \frac{1}{2}, \frac{1}{2}\right)$ & $\left(n, 0, \frac{1}{2}, \frac{1}{2}\right)$ & $q_{z} K_{d}^{a_{j} a_{i}}\left(n^{\prime} 1 \frac{1}{2}, n 0 \frac{1}{2}\right)_{1 / 2}$ \\
\hline$\left(n^{\prime}, 1, \frac{1}{2}, \frac{1}{2}\right)$ & $\left(n, 0, \frac{1}{2},-\frac{1}{2}\right)$ & $\left(q_{x}-i q_{y}\right) K_{d}^{a_{j} a_{i}}\left(n^{\prime} 1 \frac{1}{2}, n 0 \frac{1}{2}\right)_{1 / 2}$ \\
\hline$\left(n^{\prime}, 1, \frac{1}{2}, \frac{1}{2}\right)$ & $\left(n, 1, \frac{1}{2}, \frac{1}{2}\right)$ & $K_{d}^{a_{j} a_{i}}\left(n^{\prime} 1 \frac{1}{2}, n 1 \frac{1}{2}\right)_{1 / 2}$ \\
\hline$\left(n^{\prime}, 1, \frac{1}{2}, \frac{1}{2}\right)$ & $\left(n, 1, \frac{1}{2},-\frac{1}{2}\right)$ & 0 \\
\hline$\left(n^{\prime}, 1, \frac{1}{2}, \frac{1}{2}\right)$ & $\left(n, 1, \frac{3}{2}, \frac{3}{2}\right)$ & $-\frac{\sqrt{3}}{2}\left(q_{x}+i q_{y}\right) q_{z} K_{d}^{a_{j} a_{i}}\left(n^{\prime} 1 \frac{1}{2}, n 1 \frac{3}{2}\right)_{1 / 2}$ \\
\hline$\left(n^{\prime}, 1, \frac{1}{2}, \frac{1}{2}\right)$ & $\left(n, 1, \frac{3}{2}, \frac{1}{2}\right)$ & $\frac{1}{2}\left(3 q_{z}^{2}-1\right) K_{d}^{a_{j} a_{i}}\left(n^{\prime} 1 \frac{1}{2}, n 1 \frac{3}{2}\right)_{1 / 2}$ \\
\hline$\left(n^{\prime}, 1, \frac{1}{2}, \frac{1}{2}\right)$ & $\left(n, 1, \frac{3}{2},-\frac{1}{2}\right)$ & $\frac{3 q_{z}\left(q_{x}-i q_{y}\right)}{2} K_{d}^{a_{j} a_{i}}\left(n^{\prime} 1 \frac{1}{2}, n 1 \frac{3}{2}\right)_{1 / 2}$ \\
\hline$\left(n^{\prime}, 1, \frac{1}{2}, \frac{1}{2}\right)$ & $\left(n, 1, \frac{3}{2},-\frac{3}{2}\right)$ & $\frac{\sqrt{3}}{2}\left(q_{x}-i q_{y}\right)^{2} K_{d}^{a_{j} a_{i}}\left(n^{\prime} 1 \frac{1}{2}, n 1 \frac{3}{2}\right)_{1 / 2}$ \\
\hline$\left(n^{\prime}, 1, \frac{1}{2},-\frac{1}{2}\right)$ & $\left(n, 0, \frac{1}{2}, \frac{1}{2}\right)$ & $\left(q_{x}+i q_{y}\right) K_{d}^{a_{j} a_{i}}\left(n^{\prime} 1 \frac{1}{2}, n 0 \frac{1}{2}\right)_{1 / 2}$ \\
\hline$\left(n^{\prime}, 1, \frac{1}{2},-\frac{1}{2}\right)$ & $\left(n, 0, \frac{1}{2},-\frac{1}{2}\right)$ & $-q_{z} K_{d}^{a_{j} a_{i}}\left(n^{\prime} 1 \frac{1}{2}, n 0 \frac{1}{2}\right)_{1 / 2}$ \\
\hline$\left(n^{\prime}, 1, \frac{1}{2},-\frac{1}{2}\right)$ & $\left(n, 1, \frac{1}{2}, \frac{1}{2}\right)$ & 0 \\
\hline$\left(n^{\prime}, 1, \frac{1}{2},-\frac{1}{2}\right)$ & $\left(n, 1, \frac{1}{2},-\frac{1}{2}\right)$ & $K_{d}^{a_{j} a_{i}}\left(n^{\prime} 1 \frac{1}{2}, n 1 \frac{1}{2}\right)_{1 / 2}$ \\
\hline$\left(n^{\prime}, 1, \frac{1}{2},-\frac{1}{2}\right)$ & $\left(n, 1, \frac{3}{2}, \frac{3}{2}\right)$ & $-\frac{\sqrt{3}}{2}\left(q_{x}+i q_{y}\right)^{2} K_{d}^{a_{j} a_{i}}\left(n^{\prime} 1 \frac{1}{2}, n 1 \frac{3}{2}\right)_{1 / 2}$ \\
\hline$\left(n^{\prime}, 1, \frac{1}{2},-\frac{1}{2}\right)$ & $\left(n, 1, \frac{3}{2}, \frac{1}{2}\right)$ & $\frac{3\left(q_{x}+i q_{y}\right) q_{z}}{2} K_{d}^{a_{j} a_{i}}\left(n^{\prime} 1 \frac{1}{2}, n 1 \frac{3}{2}\right)_{1 / 2}$ \\
\hline$\left(n^{\prime}, 1, \frac{1}{2},-\frac{1}{2}\right)$ & $\left(n, 1, \frac{3}{2},-\frac{1}{2}\right)$ & $\frac{1-3 q_{z}^{2}}{2} K_{d}^{a_{j} a_{i}}\left(n^{\prime} 1 \frac{1}{2}, n 1 \frac{3}{2}\right)_{1 / 2}$ \\
\hline$\left(n^{\prime}, 1, \frac{1}{2},-\frac{1}{2}\right)$ & $\left(n, 1, \frac{3}{2},-\frac{3}{2}\right)$ & $-\frac{\sqrt{3}}{2}\left(q_{x}-i q_{y}\right) q_{z} K_{d}^{a_{j} a_{i}}\left(n^{\prime} 1 \frac{1}{2}, n 1 \frac{3}{2}\right)_{1 / 2}$ \\
\hline$\left(n^{\prime}, 1, \frac{3}{2}, \frac{3}{2}\right)$ & $\left(n, 0, \frac{1}{2}, \frac{1}{2}\right)$ & $-\frac{\sqrt{3}}{2}\left(q_{x}-i q_{y}\right) K_{d}^{a_{j} a_{i}}\left(n^{\prime} 1 \frac{3}{2}, n 0 \frac{1}{2}\right)_{1 / 2}$ \\
\hline$\left(n^{\prime}, 1, \frac{3}{2}, \frac{3}{2}\right)$ & $\left(n, 0, \frac{1}{2},-\frac{1}{2}\right)$ & $\begin{aligned} 2(x) & 0 \\
0 & \end{aligned}$ \\
\hline$\left(n^{\prime}, 1, \frac{3}{2}, \frac{3}{2}\right)$ & $\left(n, 1, \frac{1}{2}, \frac{1}{2}\right)$ & $-\frac{\sqrt{3}}{2}\left(q_{x}-i q_{y}\right) q_{z} K_{d}^{a_{j} a_{i}}\left(n^{\prime} 1 \frac{3}{2}, n 1 \frac{1}{2}\right)_{1 / 2}$ \\
\hline$\left(n^{\prime}, 1, \frac{3}{2}, \frac{3}{2}\right)$ & $\left(n, 1, \frac{1}{2},-\frac{1}{2}\right)$ & $-\frac{\sqrt{3}}{2}\left(q_{x}-i q_{y}\right)^{2} K_{d}^{a_{j} a_{i}}\left(n^{\prime} 1 \frac{3}{2}, n 1 \frac{1}{2}\right)_{1 / 2}$ \\
\hline$\left(n^{\prime}, 1, \frac{3}{2}, \frac{3}{2}\right)$ & $\left(n, 1, \frac{3}{2}, \frac{3}{2}\right)$ & $\frac{3\left(1-q_{z}^{2}\right)}{4} K_{d}^{a_{j} a_{i}}\left(n^{\prime} 1 \frac{3}{2}, n 1 \frac{3}{2}\right)_{1 / 2}+\frac{1+3 q_{z}^{2}}{4} K_{d}^{a_{j} a_{i}}\left(n^{\prime} 1 \frac{3}{2}, n 1 \frac{3}{2}\right)_{3 / 2}$ \\
\hline$\left(n^{\prime}, 1, \frac{3}{2}, \frac{3}{2}\right)$ & $\left(n, 1, \frac{3}{2}, \frac{1}{2}\right)$ & $\frac{\sqrt{3}}{2}\left(q_{x}-i q_{y}\right) q_{z}\left\{K_{d}^{a_{j} a_{i}}\left(n^{\prime} 1 \frac{3}{2}, n 1 \frac{3}{2}\right)_{3 / 2}-K_{d}^{a_{j} a_{i}}\left(n^{\prime} 1 \frac{3}{2}, n 1 \frac{3}{2}\right)_{1 / 2}\right\}$ \\
\hline$\left(n^{\prime}, 1, \frac{3}{2}, \frac{3}{2}\right)$ & $\left(n, 1, \frac{3}{2},-\frac{1}{2}\right)$ & $\left(q_{x}-i q_{y}\right)^{2} \frac{\sqrt{3}}{4}\left\{K_{d}^{a_{j} a_{i}}\left(n^{\prime} 1 \frac{3}{2}, n 1 \frac{3}{2}\right)_{1 / 2}-K_{d}^{a_{j} a_{i}}\left(n^{\prime} 1 \frac{3}{2}, n 1 \frac{3}{2}\right)_{3 / 2}\right\}$ \\
\hline$\left(n^{\prime}, 1, \frac{3}{2}, \frac{3}{2}\right)$ & $\left(n, 1, \frac{3}{2},-\frac{3}{2}\right)$ & 0 \\
\hline$\left(n^{\prime}, 1, \frac{3}{2}, \frac{1}{2}\right)$ & $\left(n, 0, \frac{1}{2}, \frac{1}{2}\right)$ & $q_{z} K_{d}^{a_{j} a_{i}}\left(n^{\prime} 1 \frac{3}{2}, n 0 \frac{1}{2}\right)_{1 / 2}$ \\
\hline$\left(n^{\prime}, 1, \frac{3}{2}, \frac{1}{2}\right)$ & $\left(n, 0, \frac{1}{2},-\frac{1}{2}\right)$ & $-\frac{q_{x}-i q_{y}}{2} K_{d}^{a_{j} a_{i}}\left(n^{\prime} 1 \frac{3}{2}, n 0 \frac{1}{2}\right)_{1 / 2}$ \\
\hline$\left(n^{\prime}, 1, \frac{3}{2}, \frac{1}{2}\right)$ & $\left(n, 1, \frac{1}{2}, \frac{1}{2}\right)$ & $\frac{1}{2}\left(3 q_{z}^{2}-1\right) K_{d}^{a_{j} a_{i}}\left(n^{\prime} 1 \frac{3}{2}, n 1 \frac{1}{2}\right)_{1 / 2}$ \\
\hline$\left(n^{\prime}, 1, \frac{3}{2}, \frac{1}{2}\right)$ & $\left(n, 1, \frac{1}{2},-\frac{1}{2}\right)$ & $\frac{3\left(q_{x}-i q_{y}\right) q_{z}}{2} K_{d}^{a_{j} a_{i}}\left(n^{\prime} 1 \frac{3}{2}, n 1 \frac{1}{2}\right)$ \\
\hline$\left(n^{\prime}, 1, \frac{3}{2}, \frac{1}{2}\right)$ & $\left(n, 1, \frac{3}{2}, \frac{3}{2}\right)$ & $\frac{\sqrt{3}}{2}\left(q_{x}+i q_{y}\right) q_{z}\left\{K_{d}^{a_{j} a_{i}}\left(n^{\prime} 1 \frac{3}{2}, n 1 \frac{3}{2}\right)_{3 / 2}-K_{d}^{a_{j} a_{i}}\left(n^{\prime} 1 \frac{3}{2}, n 1 \frac{3}{2}\right)_{1 / 2}\right\}$ \\
\hline
\end{tabular}


TABLE I. (Continued.)

\begin{tabular}{|c|c|c|}
\hline$\left(n^{\prime}, l^{\prime}, J^{\prime}, M^{\prime}\right)$ & $(n, l, J, M)$ & Hopping integrals $t_{n^{\prime} l^{\prime} J^{\prime} M^{\prime}, n l J M}^{a_{j} a_{i}}\left(\boldsymbol{R}_{n}+\boldsymbol{d}_{i}-\boldsymbol{d}_{j}\right)$ \\
\hline$\left(n^{\prime}, 1, \frac{3}{2}, \frac{1}{2}\right)$ & $\left(n, 1, \frac{3}{2}, \frac{1}{2}\right)$ & $\frac{3 q_{z}^{2}+1}{4} K_{d}^{a_{j} a_{i}}\left(n^{\prime} 1 \frac{3}{2}, n 1 \frac{3}{2}\right)_{1 / 2}+\frac{3\left(1-q_{z}^{2}\right)}{4} K_{d}^{a_{j} a_{i}}\left(n^{\prime} 1 \frac{3}{2}, n 1 \frac{3}{2}\right)_{3 / 2}$ \\
\hline$\left(n^{\prime}, 1, \frac{3}{2}, \frac{1}{2}\right)$ & $\left(n, 1, \frac{3}{2},-\frac{1}{2}\right)$ & 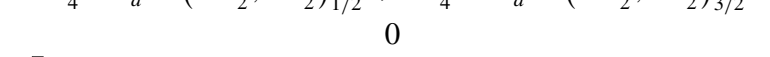 \\
\hline$\left(n^{\prime}, 1, \frac{3}{2}, \frac{1}{2}\right)$ & $\left(n, 1, \frac{3}{2},-\frac{3}{2}\right)$ & $\frac{\sqrt{3}}{4}\left(q_{x}-i q_{y}\right)^{2}\left\{K_{d}^{a_{j} a_{i}}\left(n^{\prime} 1 \frac{3}{2}, n 1 \frac{3}{2}\right)_{3 / 2}-K_{d}^{a_{j} a_{i}}\left(n^{\prime} 1 \frac{3}{2}, n 1 \frac{3}{2}\right)_{1 / 2}\right\}$ \\
\hline$\left(n^{\prime}, 1, \frac{3}{2},-\frac{1}{2}\right)$ & $\left(n, 0, \frac{1}{2}, \frac{1}{2}\right)$ & $\frac{q_{x}+i q_{y}}{2} K_{d}^{a_{j} a_{i}}\left(n^{\prime} 1 \frac{3}{2}, n 0 \frac{1}{2}\right)_{1 / 2}$ \\
\hline$\left(n^{\prime}, 1, \frac{3}{2},-\frac{1}{2}\right)$ & $\left(n, 0, \frac{1}{2},-\frac{1}{2}\right)$ & $q_{z} K_{d}^{a_{j} a_{i}}\left(n^{\prime} 1 \frac{3}{2}, n 0 \frac{1}{2}\right)_{1 / 2}$ \\
\hline$\left(n^{\prime}, 1, \frac{3}{2},-\frac{1}{2}\right)$ & $\left(n, 1, \frac{1}{2}, \frac{1}{2}\right)$ & $\frac{3 q_{z}\left(q_{x}+i q_{y}\right)}{2} K_{d}^{a_{j} a_{i}}\left(n^{\prime} 1 \frac{3}{2}, n 1 \frac{1}{2}\right)_{1 / 2}$ \\
\hline$\left(n^{\prime}, 1, \frac{3}{2},-\frac{1}{2}\right)$ & $\left(n, 1, \frac{1}{2},-\frac{1}{2}\right)$ & $\frac{1-3 q_{z}^{2}}{2} K_{d}^{a_{j} a_{i}}\left(n^{\prime} 1 \frac{3}{2}, n 1 \frac{1}{2}\right)_{1 / 2}$ \\
\hline$\left(n^{\prime}, 1, \frac{3}{2},-\frac{1}{2}\right)$ & $\left(n, 1, \frac{3}{2}, \frac{3}{2}\right)$ & $\left(q_{x}+i q_{y}\right)^{2} \frac{\sqrt{3}}{4}\left\{K_{d}^{a_{j} a_{i}}\left(n^{\prime} 1 \frac{3}{2}, n 1 \frac{3}{2}\right)_{1 / 2}-K_{d}^{a_{j} a_{i}}\left(n^{\prime} 1 \frac{3}{2}, n 1 \frac{3}{2}\right)_{3 / 2}\right\}$ \\
\hline$\left(n^{\prime}, 1, \frac{3}{2},-\frac{1}{2}\right)$ & $\left(n, 1, \frac{3}{2}, \frac{1}{2}\right)$ & 0 \\
\hline$\left(n^{\prime}, 1, \frac{3}{2},-\frac{1}{2}\right)$ & $\left(n, 1, \frac{3}{2},-\frac{1}{2}\right)$ & $\frac{3 q_{z}^{2}+1}{4} K_{d}^{a_{j} a_{i}}\left(n^{\prime} 1 \frac{3}{2}, n 1 \frac{3}{2}\right)_{1 / 2}+\frac{3\left(1-q_{z}^{2}\right)}{4} K_{d}^{a_{j} a_{i}}\left(n^{\prime} 1 \frac{3}{2}, n 1 \frac{3}{2}\right)_{3 / 2}$ \\
\hline$\left(n^{\prime}, 1, \frac{3}{2},-\frac{1}{2}\right)$ & $\left(n, 1, \frac{3}{2},-\frac{3}{2}\right)$ & $\frac{\sqrt{3}}{2}\left(q_{x}-i q_{y}\right) q_{z}\left\{K_{d}^{a_{j} a_{i}}\left(n^{\prime} 1 \frac{3}{2}, n 1 \frac{3}{2}\right)_{1 / 2}-K_{d}^{a_{j} a_{i}}\left(n^{\prime} 1 \frac{3}{2}, n 1 \frac{3}{2}\right)_{3 / 2}\right\}$ \\
\hline$\left(n^{\prime}, 1, \frac{3}{2},-\frac{3}{2}\right)$ & $\left(n, 0, \frac{1}{2}, \frac{1}{2}\right)$ & 0 \\
\hline$\left(n^{\prime}, 1, \frac{3}{2},-\frac{3}{2}\right)$ & $\left(n, 0, \frac{1}{2},-\frac{1}{2}\right)$ & $\frac{\sqrt{3}}{2}\left(q_{x}+i q_{y}\right) K_{d}^{a_{j} a_{i}}\left(n^{\prime} 1 \frac{3}{2}, n 0 \frac{1}{2}\right)_{1 / 2}$ \\
\hline$\left(n^{\prime}, 1, \frac{3}{2},-\frac{3}{2}\right)$ & $\left(n, 1, \frac{1}{2}, \frac{1}{2}\right)$ & $\frac{\sqrt{3}}{2}\left(q_{x}+i q_{y}\right)^{2} K_{d}^{a_{j} a_{i}}\left(n^{\prime} 1 \frac{3}{2}, n 1 \frac{1}{2}\right)_{1 / 2}$ \\
\hline$\left(n^{\prime}, 1, \frac{3}{2},-\frac{3}{2}\right)$ & $\left(n, 1, \frac{1}{2},-\frac{1}{2}\right)$ & $-\frac{\sqrt{3}}{2}\left(q_{x}+i q_{y}\right) q_{z} K_{d}^{a_{j} a_{i}}\left(n^{\prime} 1 \frac{3}{2}, n 1 \frac{1}{2}\right)_{1 / 2}$ \\
\hline$\left(n^{\prime}, 1, \frac{3}{2},-\frac{3}{2}\right)$ & $\left(n, 1, \frac{3}{2}, \frac{3}{2}\right)$ & 0 \\
\hline$\left(n^{\prime}, 1, \frac{3}{2},-\frac{3}{2}\right)$ & $\left(n, 1, \frac{3}{2}, \frac{1}{2}\right)$ & $\frac{\sqrt{3}}{4}\left(q_{x}+i q_{y}\right)^{2}\left\{K_{d}^{a_{j} a_{i}}\left(n^{\prime} 1 \frac{3}{2}, n 1 \frac{3}{2}\right)_{3 / 2}-K_{d}^{a_{j} a_{i}}\left(n^{\prime} 1 \frac{3}{2}, n 1 \frac{3}{2}\right)_{1 / 2}\right\}$ \\
\hline$\left(n^{\prime}, 1, \frac{3}{2},-\frac{3}{2}\right)$ & $\left(n, 1, \frac{3}{2},-\frac{1}{2}\right)$ & $\frac{\sqrt{3}}{2}\left(q_{x}+i q_{y}\right) q_{z}\left\{K_{d}^{a_{j} a_{i}}\left(n^{\prime} 1 \frac{3}{2}, n 1 \frac{3}{2}\right)_{1 / 2}-K_{d}^{a_{j} a_{i}}\left(n^{\prime} 1 \frac{3}{2}, n 1 \frac{3}{2}\right)_{3 / 2}\right\}$ \\
\hline$\left(n^{\prime}, 1, \frac{3}{2},-\frac{3}{2}\right)$ & $\left(n, 1, \frac{3}{2},-\frac{3}{2}\right)$ & $\frac{3\left(1-q_{z}^{2}\right)}{4} K_{d}^{a_{j} a_{i}}\left(n^{\prime} 1 \frac{3}{2}, n 1 \frac{3}{2}\right)_{1 / 2}+\frac{1+3 q_{z}^{2}}{4} K_{d}^{a_{j} a_{i}}\left(n^{\prime} 1 \frac{3}{2}, n 1 \frac{3}{2}\right)_{3 / 2}$ \\
\hline
\end{tabular}

much smaller than the atomic spectrum, we neglect $\Delta \bar{\varepsilon}_{n I J M}^{a_{i}, d_{i}}$ and employ the atomic spectrum calculated on the basis of the density functional theory $[38,39]$ with the local density approximation [39]. The numerical values of $\bar{\varepsilon}_{n l J}^{a_{i}}+\Delta \bar{\varepsilon}_{n l J M}^{a_{i}, d_{i}}$ and relativistic TB parameters thus-determined are listed in Tables II and III, respectively. In a later section (Sec. V), actual electronic structure calculations by means of the MFRTB method are performed by using these values, listed in Tables II and III.

\section{MFRTB METHOD}

\section{A. Matrix elements of the Hamiltonian}

We consider a crystalline material immersed in a uniform magnetic field. The electrons in the system feel not only the electric field, which is created by the periodically aligned atoms, but also the magnetic field. Let us start with the following Dirac equation for the electron [35]:

$$
H \Phi_{k}(\boldsymbol{r})=E_{k} \Phi_{k}(\boldsymbol{r})
$$

TABLE II. Numerical values of $\bar{\varepsilon}_{n l J}^{S i}+\Delta \bar{\varepsilon}_{n l J M}^{S i, \boldsymbol{d}_{i}}$ for the silicon atom.

\begin{tabular}{lc}
\hline \hline$(n, l, J, M)$ & Numerical values $(\mathrm{eV})$ \\
\hline$\left(3,0, \frac{1}{2}, \pm \frac{1}{2}\right)$ & -12.1538 \\
$\left(3,1, \frac{1}{2}, \pm \frac{1}{2}\right)$ & -5.6148 \\
$\left(3,1, \frac{3}{2}, \pm \frac{1}{2}\right),\left(3,1, \frac{3}{2}, \pm \frac{3}{2}\right)$ & -5.5853 \\
\hline \hline
\end{tabular}

with

$$
H=c \boldsymbol{\alpha} \cdot\{\boldsymbol{p}+e \boldsymbol{A}(\boldsymbol{r})\}+\beta m c^{2}+\sum_{\boldsymbol{R}_{n}} \sum_{i} v_{a_{i}}\left(\boldsymbol{r}-\boldsymbol{R}_{n}-\boldsymbol{d}_{i}\right),
$$

where $e$ is the elementary charge, and where $\boldsymbol{A}(\boldsymbol{r})$ and $v_{a_{i}}\left(\boldsymbol{r}-\boldsymbol{R}_{n}-\boldsymbol{d}_{i}\right)$ denote the vector potential of a uniform magnetic field $\boldsymbol{B}$ and the scalar potential caused by the nucleus of atom $a_{i}$, the center of which is located at $\boldsymbol{R}_{n}+\boldsymbol{d}_{i}$, respectively. The definitions of vectors $\boldsymbol{R}_{n}$ and $\boldsymbol{d}_{i}$ are the same as those below Eq. (2). We suppose that a uniform magnetic field $\boldsymbol{B}$ is applied along the $z$ axis, and that the Landau gauge is employed for $\boldsymbol{A}(\boldsymbol{r})$, i.e.,

$$
\boldsymbol{A}(\boldsymbol{r})=(0, B x, 0),
$$

TABLE III. Relativistic TB parameters between the nearestneighbor atoms for the crystalline silicon.

\begin{tabular}{cc}
\hline \hline$K_{1}\left(n^{\prime} l^{\prime} J^{\prime}, n l J\right)_{|M|}$ & Numerical values $(\mathrm{eV})$ \\
\hline$K_{1}\left(30 \frac{1}{2}, 30 \frac{1}{2}\right)_{1 / 2}$ & -1.7391 \\
$K_{1}\left(30 \frac{1}{2}, 31 \frac{1}{2}\right)_{1 / 2}$ & -1.2037 \\
$K_{1}\left(30 \frac{1}{2}, 31 \frac{3}{2}\right)_{1 / 2}$ & 1.7085 \\
$K_{1}\left(31 \frac{1}{2}, 31 \frac{1}{2}\right)_{1 / 2}$ & 0.2696 \\
$K_{1}\left(31 \frac{1}{2}, 31 \frac{3}{2}\right)_{1 / 2}$ & -1.8830 \\
$K_{1}\left(31 \frac{3}{2}, 31 \frac{3}{2}\right)_{1 / 2}$ & 1.5978 \\
$K_{1}\left(31 \frac{3}{2}, 31 \frac{3}{2}\right)_{3 / 2}$ & -1.0623 \\
\hline \hline
\end{tabular}


where $B$ is the magnitude of the magnetic field. The fourcomponent wave function for the electron in a uniform magnetic field is denoted by $\Phi_{k}(\boldsymbol{r})$ in Eq. (25), where the subscript $\boldsymbol{k}$ is the quantum number related to the magnetic Bloch theorem, which will be defined later. In order to develop the MFRTB method, $\Phi_{\boldsymbol{k}}(\boldsymbol{r})$ is expanded by using as basis functions relativistic wave functions of atoms immersed in a uniform magnetic field. The Dirac equation for an atom immersed in a uniform magnetic field and located at $\boldsymbol{R}_{n}+\boldsymbol{d}_{i}$ is given by

$$
\begin{aligned}
& {\left[c \boldsymbol{\alpha} \cdot\{\boldsymbol{p}+e \boldsymbol{A}(\boldsymbol{r})\}+\beta m c^{2}+v_{a_{i}}\left(\boldsymbol{r}-\boldsymbol{R}_{n}-\boldsymbol{d}_{i}\right)\right] \psi_{\xi}^{a_{i}, \boldsymbol{R}_{n}+\boldsymbol{d}_{i}}(\boldsymbol{r})} \\
& \quad=\varepsilon_{\xi}^{a_{i}, \boldsymbol{R}_{n}+\boldsymbol{d}_{i}} \psi_{\xi}^{a_{i}, \boldsymbol{R}_{n}+\boldsymbol{d}_{i}}(\boldsymbol{r}),
\end{aligned}
$$

where $\psi_{\xi}^{a_{i}, \boldsymbol{R}_{n}+\boldsymbol{d}_{i}}(\boldsymbol{r})$ and $\varepsilon_{\xi}^{a_{i}, \boldsymbol{R}_{n}+\boldsymbol{d}_{i}}$ denote the relativistic atomic orbital and atomic spectrum in a uniform magnetic field (27). The subscript $\xi$ in $\psi_{\xi}^{a_{i}, \boldsymbol{R}_{n}+\boldsymbol{d}_{i}}(\boldsymbol{r})$ and $\varepsilon_{\xi}^{a_{i}, \boldsymbol{R}_{n}+\boldsymbol{d}_{i}}$ is the quantum number of the atomic system. Expanding $\Phi_{\boldsymbol{k}}(\boldsymbol{r})$ in terms of $\psi_{\xi}^{a_{i}, \boldsymbol{R}_{n}+\boldsymbol{d}_{i}}(\boldsymbol{r})$, we have

$$
\Phi_{\boldsymbol{k}}(\boldsymbol{r})=\sum_{\xi} \sum_{\boldsymbol{R}_{n}} \sum_{i} C_{\boldsymbol{k}}^{\xi}\left(\boldsymbol{R}_{n}+\boldsymbol{d}_{i}\right) \psi_{\xi}^{a_{i}, \boldsymbol{R}_{n}+\boldsymbol{d}_{i}}(\boldsymbol{r}),
$$

where $C_{\boldsymbol{k}}^{\xi}\left(\boldsymbol{R}_{n}+\boldsymbol{d}_{i}\right)$ is the expansion coefficients that should be determined. Substituting Eq. (29) into Eq. (25), multiplying $\psi_{\eta}^{a_{j}, \boldsymbol{R}_{m}+\boldsymbol{d}_{j}}(\boldsymbol{r})^{\dagger}$ on both sides of Eq. (25) and integrating both sides with respect to $\boldsymbol{r}$, we get

$$
\sum_{\xi} \sum_{\boldsymbol{R}_{n}} \sum_{i} H_{\boldsymbol{R}_{m} j \eta, \boldsymbol{R}_{n} i \xi} C_{\boldsymbol{k}}^{\xi}\left(\boldsymbol{R}_{n}+\boldsymbol{d}_{i}\right)=E_{\boldsymbol{k}} C_{\boldsymbol{k}}^{\eta}\left(\boldsymbol{R}_{m}+\boldsymbol{d}_{j}\right),
$$

where

$$
H_{\boldsymbol{R}_{m} j \eta, \boldsymbol{R}_{n} i \xi}=\int \psi_{\eta}^{a_{j}, \boldsymbol{R}_{m}+\boldsymbol{d}_{j}}(\boldsymbol{r})^{\dagger} H \psi_{\xi}^{a_{i}, \boldsymbol{R}_{n}+\boldsymbol{d}_{i}}(\boldsymbol{r}) d^{3} r .
$$

Similar to the conventional [37] and relativistic TB methods (Sec. II), we use the following approximation concerning the relativistic atomic orbitals:

$$
\int \psi_{\eta}^{a_{j}, \boldsymbol{R}_{m}+\boldsymbol{d}_{j}}(\boldsymbol{r})^{\dagger} \psi_{\xi}^{a_{i}, \boldsymbol{R}_{n}+\boldsymbol{d}_{i}}(\boldsymbol{r}) d^{3} r \approx \delta_{\boldsymbol{R}_{m}, \boldsymbol{R}_{n}} \delta_{j, i} \delta_{\eta, \xi} .
$$

Equation (32) means that the overlap between the relativistic atomic orbitals centered on different atoms becomes negligible. Also, we neglect integrals involving three different centers since they are generally small compared to integrals involving two centers or the same centers. This approximation has been usually adopted in the conventional [37] and relativistic TB methods (Sec. II). Under these approximations, the Hamiltonian matrix (31) is given by

$$
\begin{aligned}
H_{\boldsymbol{R}_{m} j \eta, \boldsymbol{R}_{n} i \xi} & \\
= & \left(\varepsilon_{\xi}^{a_{i}, \boldsymbol{R}_{n}+\boldsymbol{d}_{i}}+\Delta \varepsilon_{\xi}^{a_{i}, \boldsymbol{R}_{n}+\boldsymbol{d}_{i}}\right) \delta_{\boldsymbol{R}_{m}, \boldsymbol{R}_{n}} \delta_{j, i} \delta_{\eta, \xi} \\
& +\left(1-\delta_{\boldsymbol{R}_{m}, \boldsymbol{R}_{n}} \delta_{j, i}\right) \int \psi_{\eta}^{a_{j}, \boldsymbol{R}_{m}+\boldsymbol{d}_{j}}(\boldsymbol{r})^{\dagger} \\
& \times \frac{v_{a_{j}}\left(\boldsymbol{r}-\boldsymbol{R}_{m}-\boldsymbol{d}_{j}\right)+v_{a_{i}}\left(\boldsymbol{r}-\boldsymbol{R}_{n}-\boldsymbol{d}_{i}\right)}{2} \\
& \times \psi_{\xi}^{a_{i}, \boldsymbol{R}_{n}+\boldsymbol{d}_{i}}(\boldsymbol{r}) d^{3} r,
\end{aligned}
$$

where $\Delta \varepsilon_{\xi}^{a_{i}, \boldsymbol{R}_{n}+\boldsymbol{d}_{i}}$ denotes the energy of the crystal field, which is given by [40]

$$
\begin{aligned}
& \Delta \varepsilon_{\xi}^{a_{i}, \boldsymbol{R}_{n}+\boldsymbol{d}_{i}} \\
& =\int \psi_{\xi}^{a_{i}, \boldsymbol{R}_{n}+\boldsymbol{d}_{i}}(\boldsymbol{r})^{\dagger}\left\{\sum_{\boldsymbol{R}_{m}} \sum_{k} v_{a_{k}}\left(\boldsymbol{r}-\boldsymbol{R}_{m}-\boldsymbol{d}_{k}\right)\right\} \\
& \quad \times \psi_{\xi}^{a_{m}, \boldsymbol{R}_{n}+d_{i}}(\boldsymbol{r}) d^{3} r .
\end{aligned}
$$

In order to simplify Eq. (33), let us consider the properties of $\psi_{\xi}^{a_{i}, \boldsymbol{R}_{n}+\boldsymbol{d}_{i}}(\boldsymbol{r})$ and $\varepsilon_{\xi}^{a_{i}, \boldsymbol{R}_{n}+\boldsymbol{d}_{i}}$. From Eq. (28), the Dirac equation for an atom immersed in a uniform magnetic field and located at the origin is given by

$$
\begin{aligned}
& {\left[c \boldsymbol{\alpha} \cdot\{\boldsymbol{p}+e \boldsymbol{A}(\boldsymbol{r})\}+\beta m c^{2}+v_{a_{i}}(\boldsymbol{r})\right] \psi_{\xi}^{a_{i}, \mathbf{0}}(\boldsymbol{r})} \\
& \quad=\varepsilon_{\xi}^{a_{i}, \mathbf{0}} \psi_{\xi}^{a_{i}, \mathbf{0}}(\boldsymbol{r}) .
\end{aligned}
$$

By changing the variable from $\boldsymbol{r}$ to $\boldsymbol{r}-\boldsymbol{R}_{n}-\boldsymbol{d}_{i}$, Eq. (35) is rewritten as

$$
\begin{aligned}
& {\left[c \boldsymbol{\alpha} \cdot\left\{\boldsymbol{p}+e \boldsymbol{A}\left(\boldsymbol{r}-\boldsymbol{R}_{n}-\boldsymbol{d}_{i}\right)\right\}+\beta m c^{2}+v_{a_{i}}\left(\boldsymbol{r}-\boldsymbol{R}_{n}-\boldsymbol{d}_{i}\right)\right]} \\
& \quad \times \psi_{\xi}^{a_{i}, \mathbf{0}}\left(\boldsymbol{r}-\boldsymbol{R}_{n}-\boldsymbol{d}_{i}\right) \\
& \quad=\varepsilon_{\xi}^{a_{i}, \mathbf{0}} \psi_{\xi}^{a_{i}, \mathbf{0}}\left(\boldsymbol{r}-\boldsymbol{R}_{n}-\boldsymbol{d}_{i}\right) .
\end{aligned}
$$

It should be noted that $\boldsymbol{A}\left(\boldsymbol{r}-\boldsymbol{R}_{n}-\boldsymbol{d}_{i}\right)$ yields the uniform magnetic field $\boldsymbol{B}$ as well as $\boldsymbol{A}(\boldsymbol{r})$. Therefore $\boldsymbol{A}\left(\boldsymbol{r}-\boldsymbol{R}_{n}-\boldsymbol{d}_{i}\right)$ and $\boldsymbol{A}(\boldsymbol{r})$ are related by the gauge transformation such that

$$
\boldsymbol{A}\left(\boldsymbol{r}-\boldsymbol{R}_{n}-\boldsymbol{d}_{i}\right)=\boldsymbol{A}(\boldsymbol{r})+\nabla \chi\left(\boldsymbol{r}, \boldsymbol{R}_{n}+\boldsymbol{d}_{i}\right),
$$

where $\chi\left(\boldsymbol{r}, \boldsymbol{R}_{n}+\boldsymbol{d}_{i}\right)$ is a function of both $\boldsymbol{r}$ and $\boldsymbol{R}_{n}+\boldsymbol{d}_{i}$. Substitution of Eq. (27) into Eq. (37) leads to [41]

$$
\chi\left(\boldsymbol{r}, \boldsymbol{R}_{n}+\boldsymbol{d}_{i}\right)=-B\left(R_{n x}+d_{i x}\right) y,
$$

where $R_{n x}$ and $d_{i x}$ denote the $x$-components of $\boldsymbol{R}_{n}$ and $\boldsymbol{d}_{i}$, respectively, and similar notation is used hereafter. Comparing Eq. (28) with Eq. (36), vector potentials in Eqs. (28) and (36) are different from each other by the choice of the gauge given by Eq. (37). Therefore the eigenfunctions and eigenvalues of Eqs. (28) and (36) are related by

$$
\psi_{\xi}^{a_{i}, \mathbf{0}}\left(\boldsymbol{r}-\boldsymbol{R}_{n}-\boldsymbol{d}_{i}\right)=e^{-i \frac{e}{\hbar} \chi\left(\boldsymbol{r}, \boldsymbol{R}_{n}+\boldsymbol{d}_{i}\right)} \psi_{\xi}^{a_{i}, \boldsymbol{R}_{n}+\boldsymbol{d}_{i}}(\boldsymbol{r})
$$

and

$$
\varepsilon_{\xi}^{a_{i}, \boldsymbol{0}}=\varepsilon_{\xi}^{a_{i}, \boldsymbol{R}_{n}+\boldsymbol{d}_{i}},
$$

respectively. In addition, by using Eqs. (34) and (39), we can immediately get

$$
\Delta \varepsilon_{\xi}^{a_{i}, \boldsymbol{d}_{i}}=\Delta \varepsilon_{\xi}^{a_{i}, \boldsymbol{R}_{n}+\boldsymbol{d}_{i}} .
$$

By using Eqs. (39), (40), and (41), the Hamiltonian matrix (33) is rewritten as

$$
\begin{aligned}
H_{\boldsymbol{R}_{m} j \eta, \boldsymbol{R}_{n} i \xi}= & \left(\varepsilon_{\xi}^{a_{i}, \mathbf{0}}+\Delta \varepsilon_{\xi}^{a_{i}, \boldsymbol{d}_{i}}\right) \delta_{\boldsymbol{R}_{m}, \boldsymbol{R}_{n}} \delta_{j, i} \delta_{\eta, \xi} \\
& +\left(1-\delta_{\boldsymbol{R}_{m}, \boldsymbol{R}_{n}} \delta_{j, i}\right) e^{-i \frac{e B}{\hbar}\left(R_{n x}+d_{i x}-R_{m x}-d_{j x}\right)\left(R_{m y}+d_{j y}\right)} \\
& \times T_{\eta \xi}^{a_{j} a_{i}}\left(\boldsymbol{R}_{n}-\boldsymbol{R}_{m}+\boldsymbol{d}_{i}-\boldsymbol{d}_{j}\right)
\end{aligned}
$$


with

$$
\begin{aligned}
T_{\eta \xi}^{a_{j} a_{i}} & \left(\boldsymbol{R}_{l}+\boldsymbol{d}_{i}-\boldsymbol{d}_{j}\right) \\
= & \int \psi_{\eta}^{a_{j}, \mathbf{0}}(\boldsymbol{r})^{\dagger} \frac{v_{a_{j}}(\boldsymbol{r})+v_{a_{i}}\left(\boldsymbol{r}-\boldsymbol{R}_{l}-\boldsymbol{d}_{i}+\boldsymbol{d}_{j}\right)}{2} \\
& \times \psi_{\xi}^{a_{i}, \boldsymbol{R}_{l}+\boldsymbol{d}_{i}-\boldsymbol{d}_{j}}(\boldsymbol{r}) d^{3} r .
\end{aligned}
$$

Hereafter, we refer to $T_{\eta \xi}^{a_{j} a_{i}}\left(\boldsymbol{R}_{l}+\boldsymbol{d}_{i}-\boldsymbol{d}_{j}\right)$ as the "magnetic hopping integral." By using Eq. (39), we can derive the following property for the magnetic hoping integral:

$$
\begin{aligned}
& T_{\xi \eta}^{a_{i} a_{j}}\left(-\left(\boldsymbol{R}_{n}+\boldsymbol{d}_{i}-\boldsymbol{d}_{j}\right)\right)^{*} \\
& \quad=e^{i \frac{e B}{\hbar}\left(R_{n x}+d_{i x}-d_{j x}\right)\left(R_{n y}+d_{i y}-d_{j x}\right)} T_{\eta \xi}^{a_{j} a_{i}}\left(\boldsymbol{R}_{n}+\boldsymbol{d}_{i}-\boldsymbol{d}_{j}\right) .
\end{aligned}
$$

This property is very important because it guarantees the hermicity of the Hamiltonian matrix (42). In the following section, we will approximate the magnetic hopping integral, and then Eq. (44) may work as one of the criteria of whether the approximation is physically sound or not.

\section{B. Approximation of the matrix elements}

In order to calculate the Hamiltonian matrix (42), we need both $T_{\eta \xi}^{a_{j} a_{i}}\left(\boldsymbol{R}_{l}+\boldsymbol{d}_{i}-\boldsymbol{d}_{j}\right)$ and $\varepsilon_{\xi}^{a_{i}, \boldsymbol{0}}+\Delta \varepsilon_{\xi}^{a_{i}, \boldsymbol{d}_{i}}$. For this aim, we employ the perturbation theory, where the effect of the magnetic field is treated as the perturbation. This treatment enables us to calculate both $T_{\eta \xi}^{a_{j} a_{i}}\left(\boldsymbol{R}_{l}+\boldsymbol{d}_{i}-\boldsymbol{d}_{j}\right)$ and $\varepsilon_{\xi}^{a_{i}, \mathbf{0}}+\Delta \varepsilon_{\xi}^{a_{i}, \boldsymbol{d}_{i}}$ by utilizing the atomic spectrum and relativistic hopping integrals for zero magnetic field.

$$
\text { 1. Approximation of } \varepsilon_{\xi}^{a_{i}, 0}+\Delta \varepsilon_{\xi}^{a_{i}, d_{i}}
$$

Since $\varepsilon_{\xi}^{a_{i}, \boldsymbol{0}}$ and $\Delta \varepsilon_{\xi}^{a_{i}, \boldsymbol{d}_{i}}$ are independent of the choice of the gauge, we may use the symmetric gauge for simplicity in approximating $\varepsilon_{\xi}^{a_{i}, \boldsymbol{0}}+\Delta \varepsilon_{\xi}^{a_{i}, \boldsymbol{d}_{i}}$. In the symmetric gauge, the Dirac equation for the atom is obtained by replacing $\boldsymbol{A}(\boldsymbol{r})$ with $\boldsymbol{A}_{\text {sym }}(\boldsymbol{r})$ in Eq. (35), where $\boldsymbol{A}_{\text {sym }}(\boldsymbol{r})$ is the vector potential in the symmetric gauge and is given as $\boldsymbol{B} \times \boldsymbol{r} / 2$. We shall treat the term $c e \boldsymbol{\alpha} \cdot \boldsymbol{A}_{\text {sym }}(\boldsymbol{r})$ as the perturbation. This approximation would be valid because $\psi_{\xi}^{a_{i}, \mathbf{0}}(\boldsymbol{r})$ has a large value in the vicinity of the origin, where the magnitude of $\boldsymbol{A}_{\text {sym }}(\boldsymbol{r})$ is small. The unperturbed wave function is given by $\varphi_{n l J M}^{a_{i}}(\boldsymbol{r})$, which is an eigenfunction of Eq. (5). Note that the unperturbed eigenvalue $\bar{\varepsilon}_{n l J}^{a_{i}}$ is $(2 J+1)$-fold degenerate. In order to derive the first-order perturbation energy, we shall consider the matrix elements of $c e \boldsymbol{\alpha} \cdot \boldsymbol{A}_{\mathrm{sym}}(\boldsymbol{r})$ with respect to degenerate states $\varphi_{n l J M}^{a_{i}}(\boldsymbol{r})$. They are approximately calculated as

$$
\left\langle\varphi_{n l J M^{\prime}}^{a_{i}}\left|c e \boldsymbol{\alpha} \cdot \boldsymbol{A}_{\mathrm{sym}}(\boldsymbol{r})\right| \varphi_{n l J M}^{a_{i}}\right\rangle \approx \frac{e B}{2 m} \frac{2 J+1}{2 l+1} \hbar M \delta_{M, M^{\prime}} .
$$

In the derivation of Eq. (45), the small component of $\varphi_{n l J M}^{a_{i}}(\boldsymbol{r}), i G_{n l J}^{a_{i}}(r) y_{2 J-l, J}^{M}(\theta, \phi) / r$, is approximated by $\boldsymbol{\sigma}$. $\boldsymbol{p} F_{n l J}^{a_{i}}(r) y_{l, J}^{M}(\theta, \phi) / 2 m c r$ [35]. Since the matrix of the perturbation is diagonal as shown in Eq. (45), we can readily obtain the first-order perturbation energy. This is the reason why the symmetric gauge is consciously chosen here. Within the first-order perturbation theory, $\varepsilon_{\xi}^{a_{i}, \boldsymbol{0}}$ is therefore approximated as

$$
\varepsilon_{\xi}^{a_{i}, \mathbf{0}} \approx \bar{\varepsilon}_{n l J}^{a_{i}}+\frac{e B}{2 m} \frac{2 J+1}{2 l+1} \hbar M .
$$

As mentioned before, $\psi_{\xi}^{a_{i}, \mathbf{0}}(\boldsymbol{r})$ would be well localized in the vicinity of the origin, and the magnitude of $\boldsymbol{A}_{\text {sym }}(\boldsymbol{r})$ is small around there. Therefore it would be reasonable that the relativistic atomic orbital in a uniform magnetic field, $\psi_{\xi}^{a_{i}, \boldsymbol{0}}(\boldsymbol{r})$, is approximated as the unperturbed wave function that fits on to the perturbation (zeroth-order wave function). From Eq. (45), we have

$$
\psi_{\xi}^{a_{i}, \mathbf{0}}(\boldsymbol{r})_{\mathrm{sym}} \approx \varphi_{n l J M}^{a_{i}}(\boldsymbol{r}),
$$

where the subscript "sym" denotes the atomic orbital in the symmetric gauge. By using Eqs. (34) and (47), it is also found that $\Delta \varepsilon_{\xi}^{a_{i}, \boldsymbol{d}_{i}}$ is approximated by

$$
\begin{aligned}
\Delta \varepsilon_{\xi}^{a_{i}, \boldsymbol{d}_{i}} \approx & \int \varphi_{n l J M}^{a_{i}}\left(\boldsymbol{r}-\boldsymbol{d}_{i}\right)^{\dagger}\left\{\sum_{\boldsymbol{R}_{m}} \sum_{k} v_{a_{k}}\left(\boldsymbol{r}-\boldsymbol{R}_{m}-\boldsymbol{d}_{k}\right)\right\} \\
& \times \varphi_{n l J M}^{a_{i}}\left(\boldsymbol{r}-\boldsymbol{d}_{i}\right) d^{3} r .
\end{aligned}
$$

The right-hand side of Eq. (48) is the energy of the crystal field, which is identical with $\Delta \bar{\varepsilon}_{n l J M}^{a_{i}, \boldsymbol{d}_{i}}$ from Eq. (9). Using Eqs. (9), (46), and (48), $\varepsilon_{\xi}^{a_{i}, \boldsymbol{0}}+\Delta \varepsilon_{\xi}^{a_{i}, \boldsymbol{d}_{i}}$ is thus approximated by

$$
\varepsilon_{\xi}^{a_{i}, \boldsymbol{0}}+\Delta \varepsilon_{\xi}^{a_{i}, \boldsymbol{d}_{i}} \approx \bar{\varepsilon}_{n l J}^{a_{i}}+\Delta \bar{\varepsilon}_{n l J M}^{a_{i}, \boldsymbol{d}_{i}}+\frac{e B}{2 m} \frac{2 J+1}{2 l+1} \hbar M .
$$

$$
\text { 2. Approximation of } T_{\eta \xi}^{a_{j} a_{i}}\left(R_{l}+d_{i}-d_{j}\right)
$$

In order to evaluate $T_{\eta \xi}^{a_{j} a_{i}}\left(\boldsymbol{R}_{l}+\boldsymbol{d}_{i}-\boldsymbol{d}_{j}\right)$ given by Eq. (43), we approximate both $\psi_{\eta}^{a_{j}, \boldsymbol{0}}(\boldsymbol{r})$ and $\psi_{\xi}^{a_{i}, \boldsymbol{R}_{l}+\boldsymbol{d}_{i}-\boldsymbol{d}_{j}}(\boldsymbol{r})$ by means of the lowest-order perturbation theory for degenerate states. Note that both $\psi_{\eta}^{a_{j}, \mathbf{0}}(\boldsymbol{r})$ and $\psi_{\xi}^{a_{i}, \boldsymbol{R}_{l}+\boldsymbol{d}_{i}-\boldsymbol{d}_{j}}(\boldsymbol{r})$ in Eq. (43) are the wave functions of the Landau gauge. In general, the wave function of the Landau gauge can be obtained from that of the symmetric gauge through the following transformation:

$$
\begin{gathered}
\psi_{\eta}^{a_{j}, \mathbf{0}}(\boldsymbol{r})=e^{-i \frac{e}{2 \hbar} B x y} \psi_{\eta}^{a_{j}, \mathbf{0}}(\boldsymbol{r})_{\mathrm{sym}}, \\
\psi_{\xi}^{a_{i}, \boldsymbol{R}_{l}+\boldsymbol{d}_{i}-\boldsymbol{d}_{j}}(\boldsymbol{r})=e^{-i \frac{e}{2 \hbar} B x y} \psi_{\xi}^{a_{i}, \boldsymbol{R}_{l}+\boldsymbol{d}_{i}-\boldsymbol{d}_{j}}(\boldsymbol{r})_{\mathrm{sym}},
\end{gathered}
$$

where $B x y / 2$ denotes the gauge transformation function from the symmetric gauge to the Landau gauge. Using Eqs. (47) and (50), $\psi_{\eta}^{a_{i}, \mathbf{0}}(\boldsymbol{r})$ of the Landau gauge is approximately given by

$$
\psi_{\eta}^{a_{j}, \mathbf{0}}(\boldsymbol{r}) \approx e^{-i \frac{e}{2 \hbar} B x y} \varphi_{n^{\prime} l^{\prime} J^{\prime} M^{\prime}}^{a_{j}}(\boldsymbol{r}),
$$

where $\varphi_{n^{\prime} l^{\prime} J^{\prime} M^{\prime}}^{a_{j}}(\boldsymbol{r})$ is the zero-order wave function for $\psi_{\eta}^{a_{j}, \mathbf{0}}(\boldsymbol{r})_{\mathrm{sym}}$. Furthermore, since $\varphi_{n^{\prime} l^{\prime} J^{\prime} M^{\prime}}^{a_{j}}(\boldsymbol{r})$ is localized around the origin, the phase factor $e^{-i \frac{e}{2 \hbar} B x y}$ would be approximated by 1 near the region where the magnitude of $\varphi_{n^{\prime} l^{\prime} J^{\prime} M^{\prime}}^{a_{j}}(\boldsymbol{r})$ is not negligibly small. Thus we shall approximate Eq. (52) as

$$
\psi_{\eta}^{a_{j}, \mathbf{0}}(\boldsymbol{r}) \approx \varphi_{n^{\prime} l^{\prime} J^{\prime} M^{\prime}}^{a_{j}}(\boldsymbol{r}) .
$$

Similar to Eq. (39), in the case of the symmetric gauge, $\psi_{\xi}^{a_{i}, \boldsymbol{R}_{l}+\boldsymbol{d}_{i}-\boldsymbol{d}_{j}}(\boldsymbol{r})_{\mathrm{sym}}$ is related to $\psi_{\xi}^{a_{i}, \mathbf{0}}\left(\boldsymbol{r}-\boldsymbol{R}_{l}-\boldsymbol{d}_{i}+\boldsymbol{d}_{j}\right)_{\mathrm{sym}}$ 
by

$$
\begin{aligned}
\psi_{\xi}^{a_{i}, \mathbf{0}} & \left(\boldsymbol{r}-\boldsymbol{R}_{l}-\boldsymbol{d}_{i}+\boldsymbol{d}_{j}\right)_{\mathrm{sym}} \\
= & e^{-i \frac{e B}{2 \hbar}\left\{\left(R_{l y}+d_{i y}-d_{j y}\right) x-\left(R_{l x}+d_{i x}-d_{j x}\right) y\right\}} \\
& \times \psi_{\xi}^{a_{i}, \boldsymbol{R}_{l}+\boldsymbol{d}_{i}-\boldsymbol{d}_{j}}(\boldsymbol{r})_{\mathrm{sym}},
\end{aligned}
$$

where we use $\chi\left(\boldsymbol{r}, \boldsymbol{R}_{l}+\boldsymbol{d}_{i}-\mathbf{d}_{j}\right)=$ $B\left\{\left(R_{l y}+d_{i y}-d_{j y}\right) x-\left(R_{l x}+d_{i x}-d_{j x}\right) y\right\} / 2$ for the symmetric gauge. Using Eqs. (51) and (54), we have

$$
\begin{aligned}
\psi_{\xi}^{a_{i}, \boldsymbol{R}_{l}+\boldsymbol{d}_{i}-\boldsymbol{d}_{j}}(\boldsymbol{r})= & e^{-i \frac{e}{2 \hbar} B x y} \psi_{\xi}^{a_{i}, \boldsymbol{R}_{l}+\boldsymbol{d}_{i}-\boldsymbol{d}_{j}}(\boldsymbol{r})_{\mathrm{sym}} \\
= & e^{-i \frac{e B}{2 \hbar}\left\{\left(R_{l y}+d_{i y}-d_{j y}\right) x-\left(R_{l x}+d_{i x}-d_{j x}\right) y+x y\right\}} \\
& \times \psi_{\xi}^{a_{i}, \mathbf{0}}\left(\boldsymbol{r}-\boldsymbol{R}_{l}-\boldsymbol{d}_{i}+\boldsymbol{d}_{j}\right)_{\mathrm{sym}} .
\end{aligned}
$$

Since $\quad \psi_{\xi}^{a_{i}, \mathbf{0}}\left(\boldsymbol{r}-\boldsymbol{R}_{l}-\boldsymbol{d}_{i}+\boldsymbol{d}_{j}\right)_{\mathrm{sym}} \quad$ is $\quad$ localized around $\quad \boldsymbol{r}=\boldsymbol{R}_{l}+\boldsymbol{d}_{i}-\boldsymbol{d}_{j}$, the phase factor $e^{-i \frac{e B}{2 \hbar}\left\{\left(R_{l y}+d_{i y}-d_{j y}\right) x-\left(R_{l x}+d_{i x}-d_{j x}\right) y+x y\right\}}$ would be approximated by the phase factor at $\boldsymbol{r}=\boldsymbol{R}_{l}+\boldsymbol{d}_{i}-\boldsymbol{d}_{j}$. Furthermore, using Eq. (47), we finally get

$$
\begin{aligned}
\psi_{\xi}^{a_{i}, \boldsymbol{R}_{l}+\boldsymbol{d}_{i}-\boldsymbol{d}_{j}}(\boldsymbol{r}) \approx & e^{-i \frac{e B}{2 \hbar}\left(R_{l y}+d_{i y}-d_{j y}\right)\left(R_{l x}+d_{i x}-d_{j x}\right)} \\
& \times \varphi_{n l J M}^{a_{i}}\left(\boldsymbol{r}-\boldsymbol{R}_{l}-\boldsymbol{d}_{i}+\boldsymbol{d}_{j}\right) .
\end{aligned}
$$

Substituting Eqs. (53) and (56) into Eq. (43), we obtain the approximate form of $T_{\eta \xi}^{a_{j} a_{i}}\left(\boldsymbol{R}_{l}+\boldsymbol{d}_{i}-\boldsymbol{d}_{j}\right)$ :

$$
\begin{aligned}
T_{\eta \xi}^{a_{j} a_{i}}\left(\boldsymbol{R}_{l}+\boldsymbol{d}_{i}-\boldsymbol{d}_{j}\right) \approx & e^{-i \frac{e B}{2 \hbar}\left(R_{l x}+d_{i x}-d_{j x}\right)\left(R_{l y}+d_{i y}-d_{j y}\right)} \\
& \times t_{n^{\prime} l^{\prime} J^{\prime} M^{\prime}, n l J M}^{a_{j} a_{i}}\left(\boldsymbol{R}_{l}+\boldsymbol{d}_{i}-\boldsymbol{d}_{j}\right),
\end{aligned}
$$

where $t_{n^{\prime} l^{\prime} J^{\prime} M^{\prime}, n l J M}^{a_{i} a_{i}}\left(\boldsymbol{R}_{l}+\boldsymbol{d}_{i}-\boldsymbol{d}_{j}\right)$ is the relativistic hopping integral for zero magnetic field, and is defined by Eq. (8). In this approximation, the effect of the magnetic field is stuffed into the phase factor $e^{-i \frac{e B}{2 \hbar}\left(R_{l x}+d_{i x}-d_{j x}\right)\left(R_{l y}+d_{i y}-d_{j y}\right)}$. Equation (57) is just identical with the approximation using Peierls phase $[9,13]$. That is to say, the Peierls phase is revisited by the lowest-order approximation of the perturbation theory. This means that not only is the validity of using the Peierls phase confirmed $[9,13]$, but systematical improvements beyond Eq. (57) would also be possible by incorporating the higherorder corrections of the perturbation theory into Eq. (47). In this paper, as the first trial, we shall use the approximation with Eq. (57).

Concerning Eq. (57), we also emphasize that the Hamiltonian matrix with the use of Eq. (57) remains to be a Hermitian matrix as it should be. This is easily confirmed by the fact that Eq. (57) satisfies Eq. (44).

Substitution of Eqs. (49) and (57) into Eq. (42) leads to

$$
\begin{aligned}
H_{\boldsymbol{R}_{m} j\left(n^{\prime} l^{\prime} J^{\prime} M^{\prime}\right), \boldsymbol{R}_{n} i(n l J M)}= & \left(\bar{\varepsilon}_{n l J}^{a_{i}}+\Delta \bar{\varepsilon}_{n l J M}^{a_{i}, \boldsymbol{d}_{i}}+\frac{e B}{2 m} \frac{2 J+1}{2 l+1} \hbar M\right) \delta_{\boldsymbol{R}_{m}, \boldsymbol{R}_{n}} \delta_{j, i} \delta_{n^{\prime} l^{\prime} J^{\prime} M^{\prime}, n l J M} \\
& +\left(1-\delta_{\boldsymbol{R}_{m}, \boldsymbol{R}_{n}} \delta_{j, i}\right) e^{-i \frac{e B}{2 \hbar}\left(R_{n x}+d_{i x}-R_{m x}-d_{j x}\right)\left(R_{n y}+d_{i y}+R_{m y}+d_{j y}\right)} t_{n^{\prime} l^{\prime} J^{\prime} M^{\prime}, n l J M}^{a_{j} a_{i}}\left(\boldsymbol{R}_{n}-\boldsymbol{R}_{m}+\boldsymbol{d}_{i}-\boldsymbol{d}_{j}\right) .
\end{aligned}
$$

The effects of the magnetic field are included both in the diagonal elements [the first term of Eq. (58)] as the Zeeman term and in the off-diagonal elements as the phase factor $e^{-i \frac{e B}{2 \hbar}\left(R_{l x}+d_{i x}-d_{j x}\right)\left(R_{l y}+d_{i y}-d_{j y}\right)}$. It is also found from Eq. (58) that relativistic effects are included in $\bar{\varepsilon}_{n l J}^{a_{i}}+\Delta \bar{\varepsilon}_{n l J M}^{a_{i}, \boldsymbol{d}_{i}}$ and in the relativistic hopping integral $t_{n^{\prime} l^{\prime} J^{\prime} M^{\prime}, n l J M}^{a_{1} a}\left(\boldsymbol{R}_{n}-\boldsymbol{R}_{m}+\boldsymbol{d}_{i}-\boldsymbol{d}_{j}\right)$. Substituting Eq. (58) into Eq. (30), and rewriting $\eta$ and $\xi$ by $\left(n^{\prime}, l^{\prime}, J^{\prime}, M^{\prime}\right)$ and $(n, l, J, M)$, respectively, the simultaneous equations for the expansion coefficients are given by

$$
\begin{aligned}
& \left(\bar{\varepsilon}_{n^{\prime} l^{\prime} J^{\prime}}^{a_{j}}+\Delta \bar{\varepsilon}_{n^{\prime} l^{\prime} J^{\prime} M^{\prime}}^{a_{3} \boldsymbol{d}_{j}}+\frac{e B}{2 m} \frac{2 J^{\prime}+1}{2 l^{\prime}+1} \hbar M^{\prime}\right) C_{\boldsymbol{k}}^{n^{\prime} l^{\prime} J^{\prime} M^{\prime}}\left(\boldsymbol{R}_{m}+\boldsymbol{d}_{j}\right)+\sum_{n l J M} \sum_{\boldsymbol{R}_{n}} \sum_{i}\left(1-\delta_{\boldsymbol{R}_{m}, \boldsymbol{R}_{n}} \delta_{j, i}\right) e^{-i \frac{e B}{2 \hbar}\left(R_{n x}+d_{i x}-R_{m x}-d_{j x}\right)\left(R_{n y}+d_{i y}+R_{m y}+d_{j y}\right)} \\
& \quad \times t_{n^{\prime} l^{\prime} J^{\prime} M^{\prime}, n l J M}^{a_{j} a_{i}}\left(\boldsymbol{R}_{n}-\boldsymbol{R}_{m}+\boldsymbol{d}_{i}-\boldsymbol{d}_{j}\right) C_{\boldsymbol{k}}^{n l J M}\left(\boldsymbol{R}_{n}+\boldsymbol{d}_{i}\right)=E_{\boldsymbol{k}} C_{\boldsymbol{k}}^{n^{\prime} l^{\prime} J^{\prime} M^{\prime}}\left(\boldsymbol{R}_{m}+\boldsymbol{d}_{j}\right) .
\end{aligned}
$$

Equation (59) can be rewritten by replacing the sums with respect to $\boldsymbol{R}_{n}$ and $i$ by the sum with respect to the vectors connecting the atom located at $\boldsymbol{R}_{m}+\boldsymbol{d}_{j}$ to its neighboring atoms. Since such vectors are independent of $\boldsymbol{R}_{m}$ but dependent on $\boldsymbol{d}_{j}$, they are denoted as $\boldsymbol{T}_{W}\left(\boldsymbol{d}_{j}\right)(W=1,2,3, \ldots) . W$ is numbered in the following order: $W=1,2, \ldots, W_{1}$ for the nearest-neighbor atoms, $W=W_{1}+1, W_{1}+2, \ldots, W_{2}$ for the second-nearest-neighbor atoms, $W=W_{2}+1, W_{2}+2, \ldots, W_{3}$ for the third-nearestneighbor atoms, and so on. Note that since $\boldsymbol{T}_{W}\left(\boldsymbol{d}_{j}\right)$ also denotes the vectors connecting $a_{j}$ atom to $a_{i}$ atom, $a_{i}$ varies with $W$. If we denote the dependence of $a_{i}$ on $W$ by $A(W)$, then Eq. (59) is rewritten by

$$
\begin{aligned}
& \left(\bar{\varepsilon}_{n^{\prime} l^{\prime} J^{\prime}}^{a_{j}}+\Delta \bar{\varepsilon}_{n^{\prime} l^{\prime} J^{\prime} M^{\prime}}^{a_{i} \boldsymbol{d}_{j}}+\frac{e B}{2 m} \frac{2 J^{\prime}+1}{2 l^{\prime}+1} \hbar M^{\prime}\right) C_{\boldsymbol{k}}^{n^{\prime} l^{\prime} J^{\prime} M^{\prime}}\left(\boldsymbol{R}_{m}+\boldsymbol{d}_{j}\right) \\
& \quad+\sum_{n l J M} \sum_{W} e^{-i \frac{e B}{2 \hbar} T_{W x}\left(\boldsymbol{d}_{j}\right)\left\{T_{W y}\left(\boldsymbol{d}_{j}\right)+2 R_{m y}+2 d_{j y}\right\}} t_{n^{\prime} l^{\prime} J^{\prime} M^{\prime}, n l J M}^{a_{j} A(W)}\left(\boldsymbol{T}_{W}\left(\boldsymbol{d}_{j}\right)\right) C_{\boldsymbol{k}}^{n l J M}\left(\boldsymbol{T}_{W}\left(\boldsymbol{d}_{j}\right)+\boldsymbol{R}_{m}+\boldsymbol{d}_{j}\right) \\
& =E_{\boldsymbol{k}} C_{\boldsymbol{k}}^{n^{\prime} l^{\prime} J^{\prime} M^{\prime}}\left(\boldsymbol{R}_{m}+\boldsymbol{d}_{j}\right) .
\end{aligned}
$$

Although we can obtain the coefficients $C_{\boldsymbol{k}}^{n l J M}\left(\boldsymbol{R}_{n}+\boldsymbol{d}_{i}\right)$ and $E_{\boldsymbol{k}}$ by solving the simultaneous equation [Eq. (60)], there are two problems in performing actual calculations. One is that we need the numerical values of $\left.t_{n^{\prime} l^{\prime} J^{\prime} M^{\prime}, n l J M}^{a_{j} A\left(\boldsymbol{T}_{W}\right.}\left(\boldsymbol{d}_{j}\right)\right)$.
In the previous section, we have already presented the relativistic Slater-Koster table that enables us to calculate $\left.t_{n^{\prime} l^{\prime} J^{\prime} M^{\prime}, n l J M}^{a_{A} A\left(\boldsymbol{T}_{W}\right.}\left(\boldsymbol{d}_{j}\right)\right)$. As will be shown in the subsequent sections, using the relativistic Slater-Koster table, the relativistic 
hopping integral $t_{n^{\prime} l^{\prime} J^{\prime} M^{\prime}, n l J M}^{a_{A(W)}}\left(\boldsymbol{T}_{W}\left(\boldsymbol{d}_{j}\right)\right)$ is expressed in terms of several TB parameters. TB parameters can be determined by requiring them to reproduce the electronic structure for zero magnetic field (as already mentioned in Sec. II). Another problem is that we have to solve the simultaneous equation with an infinite number of expansion coefficients. In order to overcome this problem, we employ the knowledge obtained from the translation symmetry, i.e., magnetic Bloch theorem. In Secs. IV and V, we show with specific examples that Eq. (60) is reduced to the simultaneous equation with a finite number of coefficients with the aid of the magnetic Bloch theorem.

\section{APPLICATION TO THE TWO-DIMENSIONAL SQUARE LATTICE}

In this section, we consider the two-dimensional square lattice immersed in a uniform magnetic field. We suppose that atoms have only s electrons $(l=0)$ and are located at the lattice points. It is shown that Eq. (60) is reduced to the simultaneous equation with a finite number of expansion coefficients via the magnetic Bloch theorem.

The purpose of dealing with this system is to check the validity of the MFRTB method, and to confirm the benefits of treating the relativistic effects, because this system was previously calculated with the nonrelativistic TB method by Hofstader [8], and the results are well known [8].

\section{A. Magnetic Bloch theorem}

The lattice vectors of the two-dimensional square lattice with the lattice constant $a$ are given by

$$
\begin{aligned}
\boldsymbol{R}_{n} & =n_{1} a \boldsymbol{e}_{x}+n_{2} a \boldsymbol{e}_{y} \\
& =n_{1} \boldsymbol{a}_{1}+n_{2} \boldsymbol{a}_{2},
\end{aligned}
$$

where $n_{1}$ and $n_{2}$ are integers, and $\boldsymbol{a}_{1}=a \boldsymbol{e}_{x}, \boldsymbol{a}_{2}=a \boldsymbol{e}_{y}$. The magnetic field is directed along the $z$ axis, and its magnitude $B$ is supposed to be expressed by

$$
B=\frac{2 \pi \hbar}{e a^{2}} \frac{p}{q},
$$

where $p$ and $q$ are relatively prime integers $[8,42,43]$. Let us consider a set of magnetic translation operators that commute with each other. The magnetic translation operator $U\left(\boldsymbol{R}_{n}\right)$ is defined by

$$
U\left(\boldsymbol{R}_{n}\right)=e^{i \frac{e}{\hbar} \chi\left(\boldsymbol{r}, \boldsymbol{R}_{n}\right)} T\left(\boldsymbol{R}_{n}\right),
$$

where $T\left(\boldsymbol{R}_{n}\right)$ denotes the usual translation operator given by $e^{-i \boldsymbol{R}_{n} \cdot \boldsymbol{p} / \hbar}$ [44]. It is easily shown that $U\left(\boldsymbol{R}_{n}\right)$ commutes with the Hamiltonian (26):

$$
\left[U\left(\boldsymbol{R}_{n}\right), H\right]=0 .
$$

Using Eqs. (38) and (63), the multiplication of $U\left(\boldsymbol{R}_{n}\right)$ and $U\left(\boldsymbol{R}_{m}\right)$ leads to

$$
U\left(\boldsymbol{R}_{n}\right) U\left(\boldsymbol{R}_{m}\right)=e^{-2 \pi i \frac{p}{q} m_{1} n_{2}} U\left(\boldsymbol{R}_{n}+\boldsymbol{R}_{m}\right) .
$$

Reversing the order of $\boldsymbol{R}_{n}$ and $\boldsymbol{R}_{m}$ in both sides of Eq. (65), and canceling $U\left(\boldsymbol{R}_{n}+\boldsymbol{R}_{m}\right)$ of Eq. (65) by using thus-obtained equation, we get

$$
U\left(\boldsymbol{R}_{n}\right) U\left(\boldsymbol{R}_{m}\right)=e^{-2 \pi i \frac{p}{q}\left(m_{1} n_{2}-n_{1} m_{2}\right)} U\left(\boldsymbol{R}_{m}\right) U\left(\boldsymbol{R}_{n}\right) .
$$

From this relation, if we take the set of the magnetic translation operators such as

$$
\left\{U\left(\boldsymbol{t}_{n}\right) \mid \boldsymbol{t}_{n}=n_{1} \boldsymbol{a}_{1}+q n_{2} \boldsymbol{a}_{2}\right\},
$$

then this set is shown to form an Abelian group [45]. Note that the set of translation vectors $\boldsymbol{t}_{n}$ represents a two-dimensional rectangular lattice with a unit cell of sides $a$ and $q a$. In general, the eigenfunctions of the Hamiltonian, which belong to a degenerate level, form basis functions of the irreducible representations (IRs) of the symmetry group of the Hamiltonian [46,47]. In addition, all IRs of an Abelian group are necessarily one-dimensional [47]. Therefore, eigenfunctions $\Phi_{\boldsymbol{k}}(\boldsymbol{r})$ are basis functions of IRs of the Abelian group Eq. (67), i.e., we have

$$
U\left(\boldsymbol{t}_{n}\right) \Phi_{\boldsymbol{k}}(\boldsymbol{r})=c\left(\boldsymbol{t}_{n}\right) \Phi_{\boldsymbol{k}}(\boldsymbol{r}),
$$

where $c\left(\boldsymbol{t}_{n}\right)$ is the IR of the Abelian group. The normalization condition on $c\left(\boldsymbol{t}_{n}\right) \Phi_{\boldsymbol{k}}(\boldsymbol{r})$ and Eq. (65) using $\boldsymbol{t}_{n}$ and $\boldsymbol{t}_{m}$ instead of $\boldsymbol{R}_{n}$ and $\boldsymbol{R}_{m}$ (the phase factor of the right-hand side is equal to unity) lead to

$$
c\left(\boldsymbol{t}_{n}\right)=e^{i \boldsymbol{k} \cdot \boldsymbol{t}_{n}}
$$

with a wave vector $\boldsymbol{k}$ given by

$$
\boldsymbol{k}=k_{1} \boldsymbol{b}_{1}+k_{2} \boldsymbol{b}_{2},
$$

where $k_{1}$ and $k_{2}$ are real numbers that satisfy $-1 / 2 \leqslant k_{1}<1 / 2$ and $-1 / 2 \leqslant k_{2}<1 / 2$, respectively, and where $\boldsymbol{b}_{1}$ and $\boldsymbol{b}_{2}$ are "magnetic reciprocal lattice vectors" defined as

$$
\begin{aligned}
& \boldsymbol{b}_{1}=\frac{2 \pi}{a} \boldsymbol{e}_{x} \\
& \boldsymbol{b}_{2}=\frac{2 \pi}{q a} \boldsymbol{e}_{y} .
\end{aligned}
$$

From Eqs. (70) and (71), the "magnetic first Brillouin zone" is denoted by a rectangle with length $2 \pi /(q a)$ and width $2 \pi / a$.

Using Eqs. (63), (68), and (69), we have

$$
\Psi_{\boldsymbol{k}}\left(\boldsymbol{r}-\boldsymbol{t}_{n}\right)=e^{i \boldsymbol{k} \cdot \boldsymbol{t}_{n}} e^{i \frac{e B}{\hbar} t_{n x} y} \Psi_{\boldsymbol{k}}(\boldsymbol{r}) .
$$

Equation (72) is regarded as the extension of the Bloch theorem for electrons that move in a periodic potential of the crystal and uniform magnetic field. Namely, Eq. (72) may be referred to as the "magnetic Bloch theorem." Compared to the conventional Bloch theorem, the phase factor $e^{i \frac{e B}{\hbar} t_{n x} y}$ is additionally multiplied to the right-hand side. Of course, Eq. (72) reduces to the conventional Bloch theorem when $B=0$.

\section{B. Reduction of simultaneous equations via the magnetic Bloch theorem}

From Eq. (72), we can derive the requirement fulfilled by the expansion coefficients of Eq. (29). Substituting Eq. (29) into Eq. (72), and using Eqs. (32) and (39), we get

$$
C_{\boldsymbol{k}}^{\xi}\left(\boldsymbol{t}_{n}+I \boldsymbol{a}_{2}\right)=e^{-i \boldsymbol{k} \cdot \boldsymbol{t}_{n}} C_{\boldsymbol{k}}^{\xi}\left(I \boldsymbol{a}_{2}\right)
$$

where $I=0,1, \ldots, q-1$. It should be noted that all lattice vectors $\boldsymbol{R}_{n}$ are expressed as $\boldsymbol{t}_{n}+\boldsymbol{I} \boldsymbol{a}_{2}=\left(n_{1} q+I\right) a \boldsymbol{e}_{x}+$ $n_{2} a \boldsymbol{e}_{y}$. Equation (73) means that all coefficients $C_{\boldsymbol{k}}^{\xi}\left(\boldsymbol{t}_{n}+\boldsymbol{I} \boldsymbol{a}_{2}\right)$ 
TABLE IV. Phase factors $e^{-i \frac{e B}{2 \hbar} T_{W x}\left(T_{W y}+2 I^{\prime} a\right)}$ and coefficients $C_{\boldsymbol{k}}^{n l J M}\left(I^{\prime} \boldsymbol{a}_{2}+\boldsymbol{T}_{W}\right)$ for the nearest-neighbor atoms, which appear in the left-hand side of Eq. (74).

\begin{tabular}{lccc}
\hline \hline$W$ & $\boldsymbol{T}_{W}$ & $e^{-i \frac{e B}{2 \hbar} T_{W x}\left(T_{W y}+2 I^{\prime} a\right)}$ & $C_{\boldsymbol{k}}^{n l J M}\left(I^{\prime} \boldsymbol{a}_{2}+\boldsymbol{T}_{W}\right)$ \\
\hline 1 & $(0, a)$ & 1 & $C_{\boldsymbol{k}}^{n l J M}\left(\left(I^{\prime}+1\right) \boldsymbol{a}_{2}\right)$ \\
2 & $(0,-a)$ & 1 & $C_{\boldsymbol{k}}^{n l J M}\left(\left(I^{\prime}-1\right) \boldsymbol{a}_{2}\right)$ \\
3 & $(a, 0)$ & $e^{-2 \pi i \frac{p}{q} I^{\prime}}$ & $e^{-2 \pi i k_{1}} C_{\boldsymbol{k}}^{n l J M}\left(I^{\prime} \boldsymbol{a}_{2}\right)$ \\
4 & $(-a, 0)$ & $e^{2 \pi i \frac{p}{q} I^{\prime}}$ & $e^{2 \pi i k_{1}} C_{\boldsymbol{k}}^{\text {nlJM }}\left(I^{\prime} \boldsymbol{a}_{2}\right)$ \\
\hline \hline
\end{tabular}

can be obtained by using Eq. (73) if we get $q$ coefficients $\left\{C_{\boldsymbol{k}}^{\xi}\left(I a_{2}\right) \mid I=0,1, \ldots, q-1\right\}$.

By replacing $\boldsymbol{R}_{m}$ with $\boldsymbol{t}_{m}+I^{\prime} \boldsymbol{a}_{2}\left(I^{\prime}=0,1, \ldots, q-1\right)$, and by using Eq. (73), Eq. (60) for the two-dimensional square lattice is rewritten as

$$
\begin{aligned}
& \left(\bar{\varepsilon}_{n^{\prime} l^{\prime} J^{\prime}}+\Delta \bar{\varepsilon}_{n^{\prime} l^{\prime} J^{\prime} M^{\prime}}+\frac{e B}{2 m} \frac{2 J^{\prime}+1}{2 l^{\prime}+1} \hbar M^{\prime}\right) C_{\boldsymbol{k}}^{n^{\prime} l^{\prime} J^{\prime} M^{\prime}}\left(I^{\prime} \boldsymbol{a}_{2}\right) \\
& +\sum_{n l J M} \sum_{W} e^{-i \frac{e B}{2 \hbar} T_{W x}\left(T_{W y}+2 I^{\prime} a\right)} t_{n^{\prime} l^{\prime} J^{\prime} M^{\prime}, n l J M}\left(\boldsymbol{T}_{W}\right) \\
& \times C_{\boldsymbol{k}}^{n l J M}\left(I^{\prime} \boldsymbol{a}_{2}+\boldsymbol{T}_{W}\right)=E_{\boldsymbol{k}} C_{\boldsymbol{k}}^{n^{\prime} l^{\prime} J^{\prime} M^{\prime}}\left(I^{\prime} \boldsymbol{a}_{2}\right)
\end{aligned}
$$

In deriving Eq. (74), we use the relation $e^{-i \frac{e B}{\hbar} T_{W x} t_{m y}}=1$, which is easily shown by considering the positions of the neighboring atoms. It should be noted that the same atoms are located on the lattice points in this case and therefore dependencies on $a_{i}$ and $\boldsymbol{d}_{i}$ are omitted in Eq. (74). Since the vector $\boldsymbol{I}^{\prime} \boldsymbol{a}_{2}+\boldsymbol{T}_{W}$ is generally rewritten in the form of $\boldsymbol{t}_{n^{\prime \prime}}+I^{\prime \prime} \boldsymbol{a}_{2}$, the coefficient $C_{\boldsymbol{k}}^{n l J M}\left(I^{\prime} \boldsymbol{a}_{2}+\boldsymbol{T}_{W}\right)$ of the left-hand side can be rewritten as

$$
C_{\boldsymbol{k}}^{n l J M}\left(I^{\prime} \boldsymbol{a}_{2}+\boldsymbol{T}_{W}\right)=e^{-i \boldsymbol{k} \cdot \boldsymbol{t}_{n^{\prime \prime}}} C_{\boldsymbol{k}}^{n l J M}\left(I^{\prime \prime} \boldsymbol{a}_{2}\right),
$$

by use of Eq. (73). Therefore Eq. (74) represents the simultaneous equations with a finite number of coefficients $\left\{C_{\boldsymbol{k}}^{n l J M}\left(I \boldsymbol{a}_{2}\right) \mid I=0,1, \ldots, q-1\right\}$. Namely, the simultaneous equations with an infinite number of coefficients [Eq. (60)] are simplified to those with a finite number of coefficients owing to the magnetic Bloch theorem.

We shall examine the electronic states near the Fermi level, and take into account only the relativistic hopping integral between the nearest-neighbor atoms. Since atoms have only the $s$ electrons $(l=0)$, as mentioned above, we consider the cases for $l=l^{\prime}=0, J=J^{\prime}=1 / 2, n=n^{\prime}$, and $M\left(\right.$ or $\left.M^{\prime}\right)=$ $\pm 1 / 2$ in Eq. (74). Concrete expressions for the phase factor $e^{-i \frac{e B}{2 \hbar} T_{W x}\left\{T_{W y}+2 I^{\prime} a\right\}}$ and coefficient $C_{\boldsymbol{k}}^{n l J M}\left(I^{\prime} \boldsymbol{a}_{2}+\boldsymbol{T}_{W}\right)$ in the left-hand side of Eq. (74) are given in Table IV. Furthermore, using the relativistic Slater-Koster table (Table I), Eq. (74) is rewritten as

$$
\begin{gathered}
\left\{\bar{\varepsilon}_{n^{\prime} 0 \frac{1}{2}}+\Delta \bar{\varepsilon}_{n^{\prime} 0 \frac{1}{2} M^{\prime}}+\frac{e B}{m} \hbar M^{\prime}+2 K_{1}\left(n^{\prime} 0 \frac{1}{2}, n^{\prime} 0 \frac{1}{2}\right)_{1 / 2} \cos \left[2 \pi\left(\frac{p}{q} I^{\prime}+k_{1}\right)\right]\right\} C_{\boldsymbol{k}}^{n^{\prime} 0 \frac{1}{2} M^{\prime}}\left(I^{\prime} \boldsymbol{a}_{2}\right) \\
+K_{1}\left(n^{\prime} 0 \frac{1}{2}, n^{\prime} 0 \frac{1}{2}\right)_{1 / 2}\left[C_{\boldsymbol{k}}^{n^{\prime} 0 \frac{1}{2} M^{\prime}}\left(\left(I^{\prime}+1\right) \boldsymbol{a}_{2}\right)+C_{\boldsymbol{k}}^{n^{\prime} 0 \frac{1}{2} M^{\prime}}\left(\left(I^{\prime}-1\right) \boldsymbol{a}_{2}\right)\right]=E_{\boldsymbol{k}} C_{\boldsymbol{k}}^{n^{\prime} 0 \frac{1}{2} M^{\prime}}\left(I^{\prime} \boldsymbol{a}_{2}\right) .
\end{gathered}
$$

It should be noted that due to Eq. (73), $C_{\boldsymbol{k}}^{n^{\prime} 0 \frac{1}{2} M^{\prime}}\left(\left(I^{\prime}+1\right) \boldsymbol{a}_{2}\right)$ and $C_{\boldsymbol{k}}^{n^{\prime} 0 \frac{1}{2} M^{\prime}}\left(\left(I^{\prime}-1\right) \boldsymbol{a}_{2}\right)$ in Eq. (76) are equal to

$$
\begin{aligned}
& C_{\boldsymbol{k}}^{n^{\prime} 0 \frac{1}{2} M^{\prime}}\left(\left(I^{\prime}+1\right) \boldsymbol{a}_{2}\right)= \begin{cases}e^{-2 \pi i k_{2}} C_{\boldsymbol{k}}^{n^{\prime} 0 \frac{1}{2} M^{\prime}}(0) & \text { for } \quad I^{\prime}=q-1, \\
C_{\boldsymbol{k}}^{n^{\prime} 0 \frac{1}{2} M^{\prime}}\left(\left(I^{\prime}+1\right) \boldsymbol{a}_{2}\right) & \text { for } \quad I^{\prime} \neq q-1,\end{cases} \\
& C_{\boldsymbol{k}}^{n^{\prime} 0 \frac{1}{2} M^{\prime}}\left(\left(I^{\prime}-1\right) \boldsymbol{a}_{2}\right)= \begin{cases}e^{2 \pi i k_{2}} C_{\boldsymbol{n ^ { \prime }} 0 \frac{1}{2} M^{\prime}}\left((q-1) a_{2}\right) & \text { for } I^{\prime}=0, \\
C_{\boldsymbol{k}}^{n^{\prime} 0 \frac{1}{2} M^{\prime}}\left(\left(I^{\prime}-1\right) \boldsymbol{a}_{2} s\right) & \text { for } I^{\prime} \neq 0, a\end{cases}
\end{aligned}
$$

respectively. It is found from Eqs. (77) and (78) that Eq. (76) represents the simultaneous equations for $2 q$ coefficients $\left\{C_{\boldsymbol{k}}^{n 0 \frac{1}{2} M}\left(I \boldsymbol{a}_{2}\right) \mid I=0,1, \ldots, q-1, M= \pm 1 / 2\right\}$.

If we neglect the Zeeman term, then Eq. (76) is reduced to an equivalent form to that of Hofstadter [8]. Actually, as can be seen in the next section, Sec. IV C, the calculation results are identical with those of Hofstadter [8] in the case of no Zeeman term.

\section{Calculation results}

The energy eigenvalues for the two-dimensional square lattice are calculated on the basis of Eq. (76). Since the qualitative shapes of the energy dispersion and diagram are not dependent on the choice of the values of $\bar{\varepsilon}_{n 0 \frac{1}{2}}+\Delta \bar{\varepsilon}_{n 0 \frac{1}{2} M}$ and $K_{1}\left(n 0 \frac{1}{2}, n 0 \frac{1}{2}\right)_{1 / 2}$, they are taken from those for the crystalline silicon, which are already listed in Tables II and III. Figure 1 shows the energy dispersion $E_{k}$ for the two-dimensional square lattice in the presence of the uniform magnetic field, where $p$ and $q$ are fixed at 40 and 401, respectively. The inset of Fig. 1 is the magnified view. The labels indicated in the horizontal axis of Fig. 1 denote the special $\boldsymbol{k}$ points in the magnetic first Brillouin zone, which is shown in Fig. 2. It is found from Fig. 1 that $E_{\boldsymbol{k}}$ depends little on $\boldsymbol{k}$, while the band dispersion for the zero magnetic field case ordinarily depends on $\boldsymbol{k}$ because it is written as the cosine curve with an amplitude of $2\left|K_{1}\left(30 \frac{1}{2}, 30 \frac{1}{2}\right)_{1 / 2}\right|=3.4782 \mathrm{eV}$. By applying the magnetic field, the orbital motion of electrons in the plane perpendicular to the magnetic field is essentially changed corresponding to the quantization of the orbital motion of electrons in the magnetic field. As a result, $E_{\boldsymbol{k}}$ curves become nearly flat as shown 


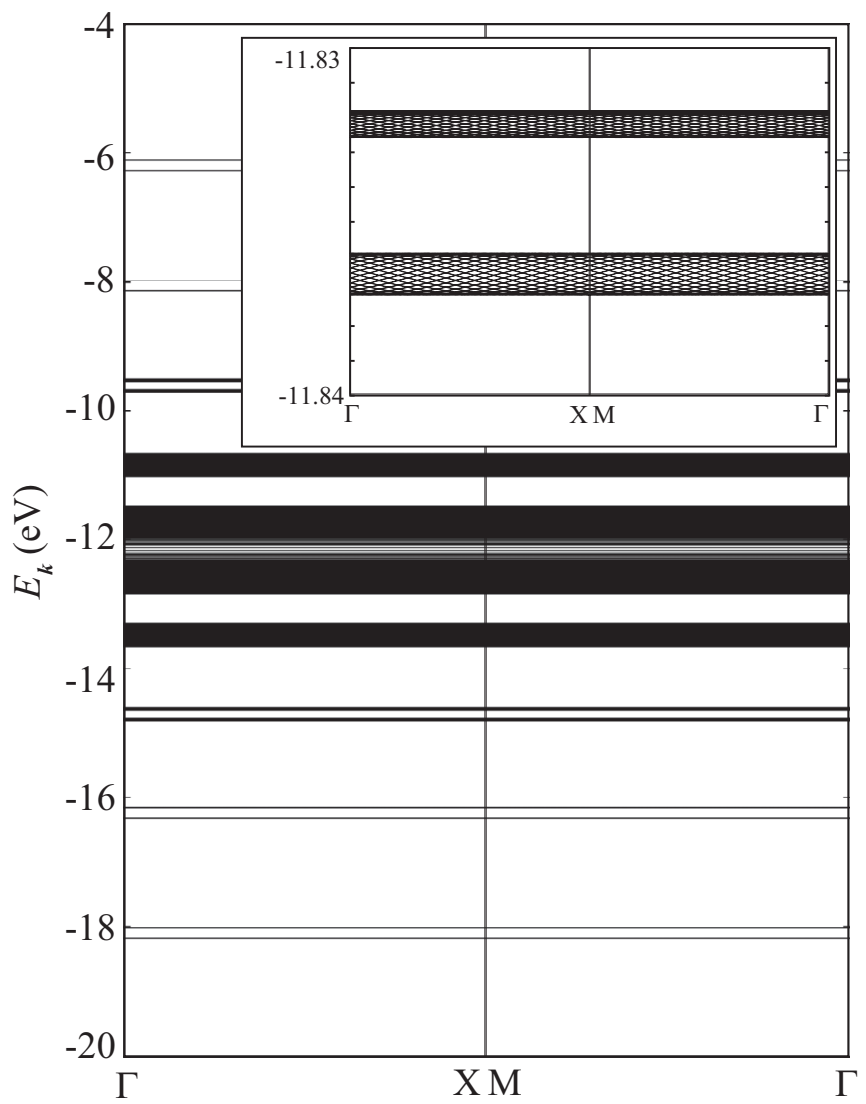

FIG. 1. Energy dispersion for a two-dimensional square lattice immersed in a uniform magnetic field. Values of $p$ and $q$ are 40 and 401 , respectively. The inset is the magnified view. The labels in the horizontal axis denote the special $\boldsymbol{k}$ points that are indicated in Fig. 2.

in Fig. 1. The bandwidths of these flat bands are obviously dependent on the magnitude of the magnetic field. The widths increase as the magnetic field becomes large, as shown in Figs. 3(a)-3(c). This behavior can be observed also in the crystalline silicon, which will be in the next section. Detailed discussions on this bandwidth will be developed in Sec. V D.

The magnetic-field-dependent energy diagrams are calculated with and without the Zeeman term in Eq. (76). The results with and without the Zeeman term are shown in Figs. 4(a) and 4(b), respectively, where all values of $E_{\boldsymbol{k}}$ for $\boldsymbol{k}$ 's that correspond to the horizontal axis of Fig. 1 are plotted in each magnetic field. In these calculations, $p$ changes from

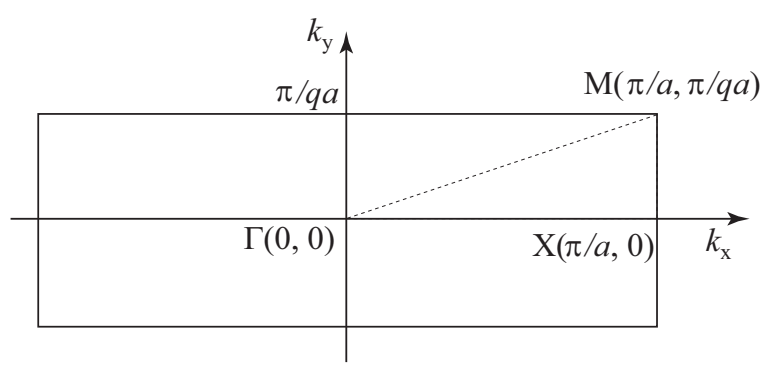

FIG. 2. The magnetic first Brillouin zone for a two-dimensional square lattice immersed in a uniform magnetic field.

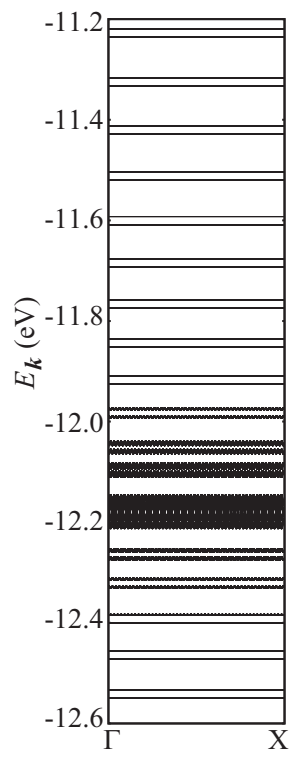

(a) $p / q=1 / 101$

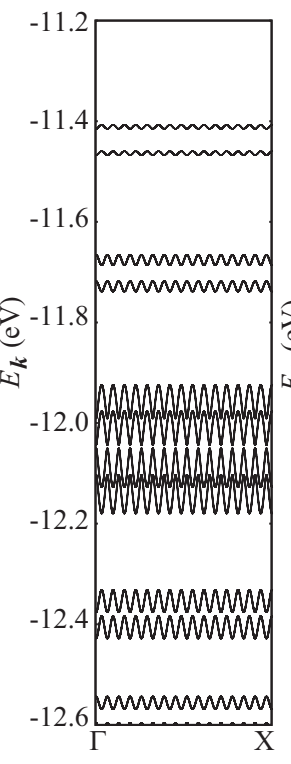

(b) $p / q=1 / 31$

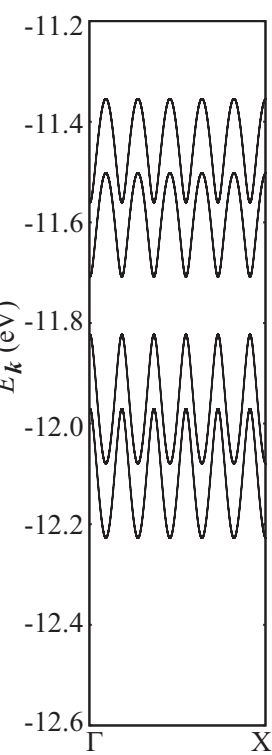

(c) $p / q=1 / 11$
FIG. 3. Magnetic-field dependence of the energy dispersion for a two-dimensional square lattice immersed in a uniform magnetic field. Energy dispersions in the cases of (a) $p / q=1 / 101$, (b) $p / q=1 / 31$, and (c) $p / q=1 / 11$.

1 to 401 with fixing $q$ at 401 . Due to the Zeeman term of Eq. (76), the energy diagram shown in Fig. 4(a) splits into two parts. It is also confirmed that the magnitude of the band splitting becomes large as the magnetic field increases. As can be seen in Fig. 4(b), the Hofstadter butterfly diagram [8] is reproduced in the case of no Zeeman term as is expected from Eq. (76). Namely, Fig. 4(b) has the properties of the Butterfly diagram, which are shown by D. Hofstadter [8]. For example, the spectrum for $p / q$ is identical with the spectra for $p / q+n$ ( $n$ : integers) and $-p / q$ [8]. Thus the present MFRTB method is recognized as a generalized method that includes the Hofstadter method [8].

\section{APPLICATION TO A CRYSTALLINE SILICON IMMERSED IN A MAGNETIC FIELD}

In this section, we shall apply the MFRTB method to a crystalline silicon immersed in a magnetic field. Similar to the case of a two-dimensional square lattice (Sec. IV), nearly flat $E_{\boldsymbol{k}}$ curves and a magnetic-field dependence of their bandwidths are observed also for this system. In addition, as mentioned in Sec. I, the present MFRTB method suggests a way to determine a formula of the magnetic field that is consistent with the magnetic Bloch theorem. With emphasis on these points, we present the electronic structure of a crystalline silicon immersed in a magnetic field.

\section{A. Magnetic Bloch theorem for the crystalline silicon}

Crystalline silicon has the diamond structure, the lattice of which is the face-centered cubic (FCC). The translation vectors of the diamond structure with the lattice constant $a$ are given by

$$
\boldsymbol{R}_{n}=n_{1} \boldsymbol{a}_{1}+n_{2} \boldsymbol{a}_{2}+n_{3} \boldsymbol{a}_{3},
$$




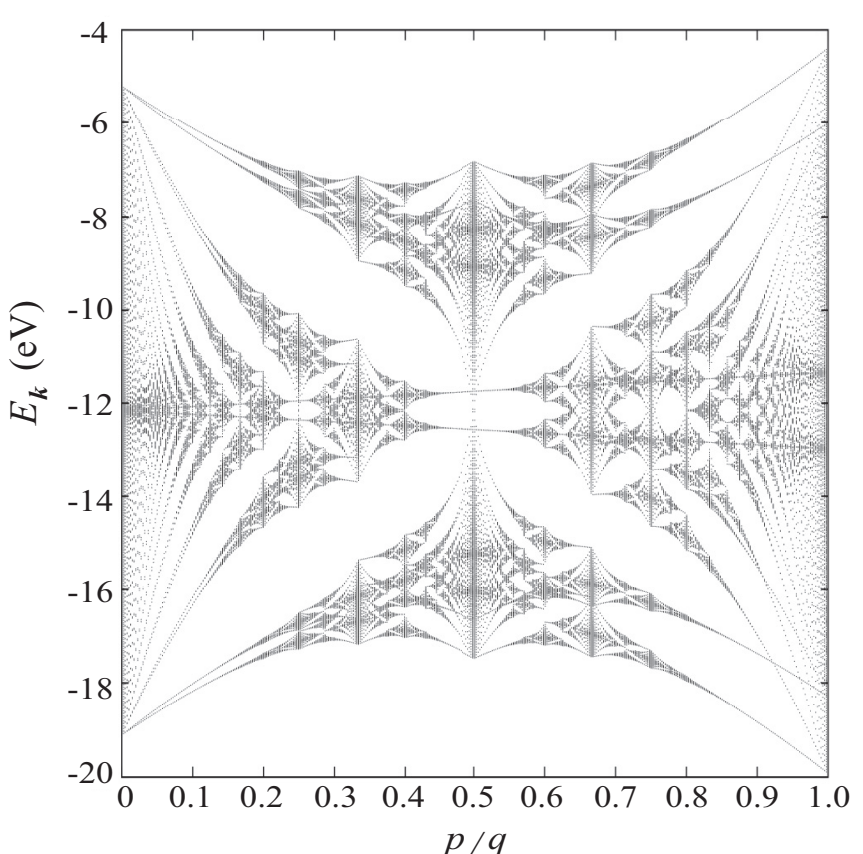

(a)

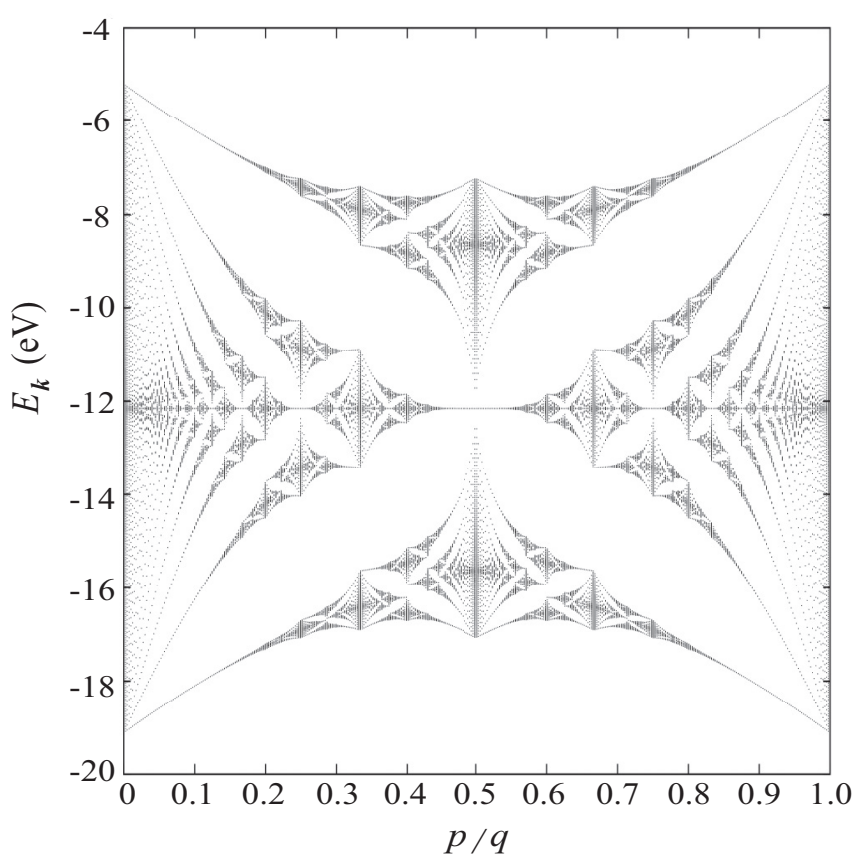

(b)

FIG. 4. (a) Dependence of the energy diagram on the magnetic field for the two-dimensional square lattice. (b) Hofstadter butterfly diagram calculated on the basis of the MFRTB method neglecting the Zeeman term.

where

$$
\begin{aligned}
\boldsymbol{a}_{1} & =\frac{a}{2}\left(\boldsymbol{e}_{x}+\boldsymbol{e}_{y}\right), \\
\boldsymbol{a}_{2} & =\frac{a}{2}\left(\boldsymbol{e}_{x}+\boldsymbol{e}_{z}\right), \\
\boldsymbol{a}_{3} & =\frac{a}{2}\left(\boldsymbol{e}_{y}+\boldsymbol{e}_{z}\right)
\end{aligned}
$$

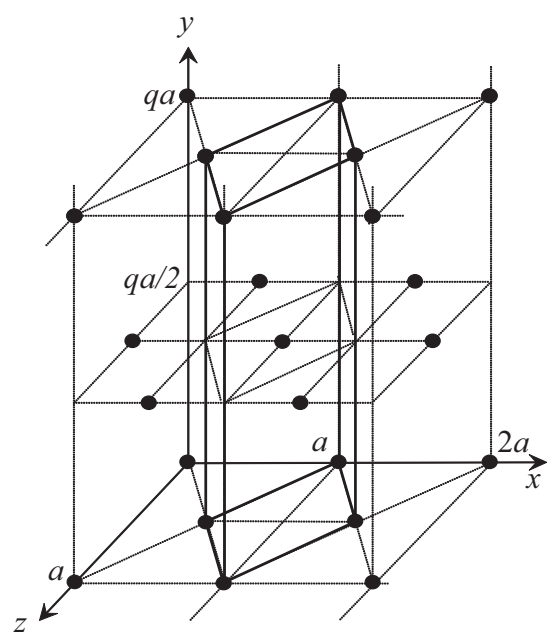

(a)

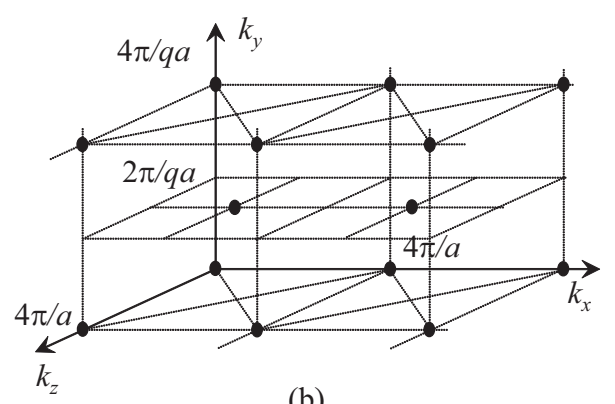

(b)

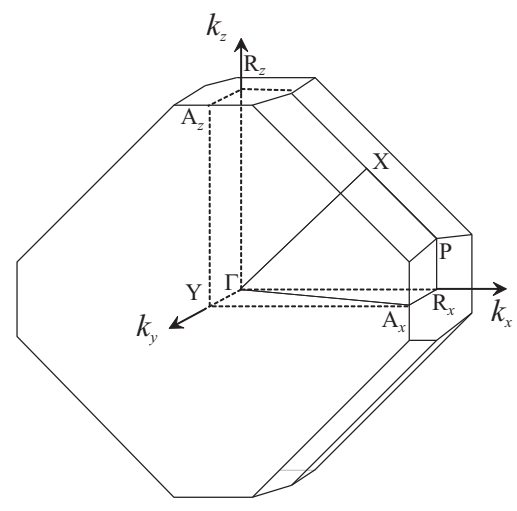

$\Gamma:(0,0,0)$

$\mathrm{X}: \frac{2 \pi}{a}\left(\frac{1}{2}+\frac{1}{4 q^{2}}, 0, \frac{1}{2}+\frac{1}{4 q^{2}}\right)$

$\mathrm{P}: \frac{2 \pi}{a}\left(1,0, \frac{1+\sqrt{2}}{2 q^{2}}\right)$

$\mathrm{R}_{x}: \frac{2 \pi}{a}(1,0,0)$

$\mathrm{A}_{x}: \frac{2 \pi}{a}\left(1, \frac{1}{q}, 0\right)$

(c)

FIG. 5. (a) Schematic view of the lattice defined by the translation vectors $\boldsymbol{t}_{n}\left(=n_{1} q \boldsymbol{a}_{1}+n_{2} \boldsymbol{a}_{2}+n_{3} \boldsymbol{a}_{3}^{\prime}\right)$. (b) Schematic view of the reciprocal lattice spanned by Eq. (87). (c) Schematic view of the magnetic first Brillouin zone for the crystalline silicon immersed in a magnetic field directed along the $z$ axis.

are the primitive translation vectors, and where $n_{1}, n_{2}$, and $n_{3}$ are integers. Two silicon atoms are located at $\boldsymbol{d}_{1}=0$ and $\boldsymbol{d}_{2}=a\left(\boldsymbol{e}_{x}+\boldsymbol{e}_{y}+\boldsymbol{e}_{z}\right) / 4$ from each lattice point, respectively. Suppose that the magnetic field is directed along the $z$ axis, and that its magnitude $B$ is given by [48]

$$
B=\frac{16 \pi \hbar}{e a^{2}} \frac{p}{q},
$$


where $p$ and $q$ are relatively prime integers, and $q$ is supposed to be a primitive number that is larger than 2 . This form is introduced so that the solution of Eq. (60) is consistent with the magnetic Bloch theorem. This point will be discussed later.

Similar to the case of the two-dimensional square lattice, let us consider a set of magnetic translation operators that commute with each other. By using Eqs. (38), (63), (79), and (81), the multiplication of two magnetic translation operators is given by

$$
U\left(\boldsymbol{R}_{n}\right) U\left(\boldsymbol{R}_{m}\right)=e^{-2 \pi i \frac{2 p}{q}\left(m_{1}+m_{2}\right)\left(n_{1}+n_{3}\right)} U\left(\boldsymbol{R}_{n}+\boldsymbol{R}_{m}\right) .
$$

Reversing the order of $\boldsymbol{R}_{n}$ and $\boldsymbol{R}_{m}$ in both sides of Eq. (82), and canceling $U\left(\boldsymbol{R}_{n}+\boldsymbol{R}_{m}\right)$ of Eq. (82) by using thus-obtained equation, we get

$$
\begin{aligned}
U\left(\boldsymbol{R}_{n}\right) U\left(\boldsymbol{R}_{m}\right)= & e^{-2 \pi i \frac{2 p}{q}\left\{\left(m_{1}+m_{2}\right)\left(n_{1}+n_{3}\right)-\left(m_{1}+m_{3}\right)\left(n_{1}+n_{2}\right)\right\}} \\
& \times U\left(\boldsymbol{R}_{m}\right) U\left(\boldsymbol{R}_{n}\right) .
\end{aligned}
$$

Using Eq. (83), we can easily find what set of the translation operators forms the Abelian group. Specifically, the following set forms the Abelian group

$$
\left\{U\left(\boldsymbol{t}_{n}\right) \mid \boldsymbol{t}_{n}=n_{1} q \boldsymbol{a}_{1}+n_{2} \boldsymbol{a}_{2}+n_{3} \boldsymbol{a}_{3}^{\prime}\right\},
$$

where $\boldsymbol{a}_{3}^{\prime}=a\left(\boldsymbol{e}_{z}-\boldsymbol{e}_{x}\right) / 2$. It is also shown that Eq. (84) has the smallest period of the translation among the operator sets that form the Abelian groups. Note that the set of three vectors $\boldsymbol{a}_{1}, \boldsymbol{a}_{2}$, and $\boldsymbol{a}_{3}^{\prime}$ is one of the choices of the primitive translation vectors of the FCC lattice as well as the set of $\boldsymbol{a}_{1}, \boldsymbol{a}_{2}$, and $\boldsymbol{a}_{3}$. The set of lattice points $\left\{\boldsymbol{t}_{n}\right\}$ forms the body-centered tetragonal lattice, which is extended along the $y$ axis as shown in Fig. 5(a).

According to the general discussion on the relation between the eigenfunctions of the Hamiltonian and the basis functions of IRs of the symmetry group [47], we can say that the eigenfunctions of the Hamiltonian, which are denoted by $\Phi_{k}(\boldsymbol{r})$, can be the basis functions of IRs of Eq. (84). In a similar way to the case of the two-dimensional square lattice, we have

$$
U\left(\boldsymbol{t}_{n}\right) \Phi_{\boldsymbol{k}}(\boldsymbol{r})=e^{i \boldsymbol{k} \cdot \boldsymbol{t}_{n}} \Phi_{\boldsymbol{k}}(\boldsymbol{r}),
$$

with the wave vector $\boldsymbol{k}$ given by

$$
\boldsymbol{k}=k_{1} \boldsymbol{b}_{1}+k_{2} \boldsymbol{b}_{2}+k_{3} \mathbf{b}_{3},
$$

where $k_{1}, k_{2}$, and $k_{3}$ are real numbers that take $-\frac{1}{2} \leqslant k_{1}, k_{2}, k_{3}<\frac{1}{2}$, and where $\boldsymbol{b}_{1}, \boldsymbol{b}_{2}$, and $\boldsymbol{b}_{3}$ are magnetic reciprocal lattice vectors for the crystalline silicon, which are defined as

$$
\begin{aligned}
& \boldsymbol{b}_{1}=\frac{4 \pi}{a q} \boldsymbol{e}_{y}, \\
& \boldsymbol{b}_{2}=\frac{2 \pi}{a}\left(\boldsymbol{e}_{x}-\boldsymbol{e}_{y}+\boldsymbol{e}_{z}\right), \\
& \boldsymbol{b}_{3}=\frac{2 \pi}{a}\left(-\boldsymbol{e}_{x}+\boldsymbol{e}_{y}+\boldsymbol{e}_{z}\right) .
\end{aligned}
$$

The reciprocal lattice spanned by Eq. (87) and the corresponding magnetic first Brillouin zone are given in Figs. 5(b) and 5(c), respectively.

Equations (38), (63), and (85) lead to the magnetic Bloch theorem for the crystalline silicon:

$$
\Phi_{\boldsymbol{k}}\left(\boldsymbol{r}-\boldsymbol{t}_{n}\right)=e^{i \boldsymbol{k} \cdot \boldsymbol{t}_{n}} e^{i \frac{e B}{\hbar} t_{n x} y} \Phi_{\boldsymbol{k}}(\boldsymbol{r}) .
$$

This theorem is used in the next section to reduce the order of the simultaneous equations (60) to finite.

\section{B. Reduction of simultaneous equations via the magnetic Bloch theorem}

The lattice vectors $\boldsymbol{R}_{n}$ are expressed by

$$
\boldsymbol{t}_{n}+\boldsymbol{I} \boldsymbol{a}_{1}=\left(n_{1} q+I\right) \boldsymbol{a}_{1}+n_{2} \boldsymbol{a}_{2}+n_{3} \boldsymbol{a}_{3}{ }_{3},
$$

where $I=0,1, \ldots, q-1$. Similar to the case of the twodimensional square lattice, substituting Eq. (29) into Eq. (88), and using Eqs. (39) and (89), we have

$$
\sum_{\xi} \sum_{\boldsymbol{t}_{n}} \sum_{I=0}^{q-1} \sum_{i}\left\{C_{\boldsymbol{k}}^{\xi}\left(\boldsymbol{t}_{n}+I \boldsymbol{a}_{1}+\boldsymbol{d}_{i}-\boldsymbol{t}_{m}\right)-e^{i \boldsymbol{k} \cdot \boldsymbol{t}_{m}} C_{\boldsymbol{k}}^{\xi}\left(\boldsymbol{t}_{n}+I \boldsymbol{a}_{1}+\boldsymbol{d}_{i}\right)\right\} \psi_{\xi}^{a_{i}, \boldsymbol{t}_{n}+I \boldsymbol{a}_{1}+\boldsymbol{d}_{i}}(\boldsymbol{r})=0 .
$$

Utilizing the orthonormality of the basis functions, i.e., Eq. (32), a relation between the expansion coefficients is obtained:

$$
C_{\boldsymbol{k}}^{\xi}\left(\boldsymbol{t}_{m}+I \boldsymbol{a}_{1}+\boldsymbol{d}_{i}\right)=e^{-i \boldsymbol{k} \cdot \boldsymbol{t}_{m}} C_{\boldsymbol{k}}^{\xi}\left(I \boldsymbol{a}_{1}+\boldsymbol{d}_{i}\right) .
$$

Equation (91) is an alternative expression of the magnetic Bloch theorem.

Rewriting Eq. (60) by using $\boldsymbol{t}_{m}+I^{\prime} \boldsymbol{a}_{1}$ instead of $\boldsymbol{R}_{m}$, and substituting Eq. (91) into this, we have

$$
\begin{aligned}
& \left(\bar{\varepsilon}_{n^{\prime} l^{\prime} J^{\prime}}^{a_{j}}+\Delta \bar{\varepsilon}_{n^{\prime} l^{\prime} J^{\prime} M^{\prime}}^{a_{j}, \boldsymbol{d}_{j}}+\frac{e B}{2 m} \frac{2 J^{\prime}+1}{2 l^{\prime}+1} \hbar M^{\prime}\right) C_{\boldsymbol{k}}^{n^{\prime} l^{\prime} J^{\prime} M^{\prime}}\left(I^{\prime} \boldsymbol{a}_{1}+\boldsymbol{d}_{j}\right)+\sum_{n l J M} \sum_{W} e^{-i \frac{e B}{2 \hbar} T_{W x}\left(\boldsymbol{d}_{j}\right)\left[T_{W y}\left(\boldsymbol{d}_{j}\right)+2 t_{m y}+2 I^{\prime} a_{1 y}+2 d_{j y}\right]} \\
& \quad \times t_{n^{\prime} l^{\prime} J^{\prime} M^{\prime}, n l J M}\left(\boldsymbol{T}_{W}\left(\boldsymbol{d}_{j}\right)\right) C_{\boldsymbol{k}}^{n l J M}\left(\boldsymbol{T}_{W}\left(\boldsymbol{d}_{j}\right)+\boldsymbol{I}^{\prime} \boldsymbol{a}_{1}+\boldsymbol{d}_{j}\right)=E_{\boldsymbol{k}} C_{\boldsymbol{k}}^{n^{\prime} l^{\prime} J^{\prime} M^{\prime}}\left(I^{\prime} \boldsymbol{a}_{1}+\boldsymbol{d}_{j}\right),
\end{aligned}
$$

where we remove the superscripts of the hopping integral since all sites are, of course, occupied by the silicon atoms. It should be noticed that since $\boldsymbol{T}_{W}\left(\boldsymbol{d}_{j}\right)+I^{\prime} \boldsymbol{a}_{1}+\mathbf{d}_{j}$ denotes the position of Si atom, this vector is rewritten in the form of $\boldsymbol{t}_{n^{\prime}}+I^{\prime \prime} \boldsymbol{a}_{1}+\boldsymbol{d}_{i^{\prime}}$. Therefore $C_{\boldsymbol{k}}^{n l J M}\left(\boldsymbol{T}_{W}\left(\boldsymbol{d}_{j}\right)+I^{\prime} \boldsymbol{a}_{1}+\boldsymbol{d}_{j}\right)$ in the left-hand side of Eq. (92) is rewritten as

$$
C_{\boldsymbol{k}}^{n l J M}\left(\boldsymbol{T}_{W}\left(\boldsymbol{d}_{j}\right)+I^{\prime} \boldsymbol{a}_{1}+\boldsymbol{d}_{j}\right)=e^{-i \boldsymbol{k} \cdot \boldsymbol{t}_{n^{\prime}}} C_{\boldsymbol{k}}^{n l J M}\left(I^{\prime \prime} \boldsymbol{a}_{1}+\boldsymbol{d}_{i^{\prime}}\right),
$$

where Eq. (91) is used. Equations (92) and (93) mean the reduction of the simultaneous equation (60). That is to say, they are regarded as the simultaneous equations for the finite number of coefficients $\left\{C_{\boldsymbol{k}}^{n l J M}\left(\mathrm{Ia}_{1}+\boldsymbol{d}_{i}\right) \mid I=0,1,2, \ldots, q-1 ; i=1,2 ; n l J M\right\}$. 
TABLE V. Phase factors $e^{-i \frac{e B}{2 \hbar} T_{W x}\left(\boldsymbol{d}_{1}\right)\left(T_{W y}\left(\boldsymbol{d}_{1}\right)+2 I^{\prime} a_{1 y}+2 d_{1 y}\right)}$ and coefficients $C_{\boldsymbol{k}}^{n l J M}\left(\boldsymbol{T}_{W}\left(\boldsymbol{d}_{1}\right)+I^{\prime} \boldsymbol{a}_{1}+\boldsymbol{d}_{1}\right)$ that appear in the left-hand side of Eq. (95).

\begin{tabular}{lccc}
\hline \hline$W$ & $\boldsymbol{T}_{W}\left(\boldsymbol{d}_{1}\right)$ & $e^{-i \frac{e B}{2 \hbar} T_{W x}\left(\boldsymbol{d}_{1}\right)\left(T_{W y}\left(\boldsymbol{d}_{1}\right)+I^{\prime} a_{1 y}+2 d_{1 y}\right)}$ & $C_{\boldsymbol{k}}^{n l J M}\left(\boldsymbol{T}_{W}\left(\boldsymbol{d}_{1}\right)+I^{\prime} \boldsymbol{a}_{1}+\boldsymbol{d}_{1}\right)$ \\
\hline 1 & $\frac{a}{4}(1,1,1)$ & $e^{-2 \pi i \frac{p}{q}\left(I^{\prime}+\frac{1}{4}\right)}$ & $C_{\boldsymbol{k}}^{n l J M}\left(I^{\prime} \boldsymbol{a}_{1}+\boldsymbol{d}_{2}\right)$ \\
2 & $\frac{a}{4}(1,-1,-1)$ & $e^{-2 \pi i \frac{p}{q}\left(I^{\prime}-\frac{1}{4}\right)}$ & $e^{2 \pi i k_{3}} C_{\boldsymbol{k}}^{n l J M}\left(\left(I^{\prime}-1\right) \boldsymbol{a}_{1}+\boldsymbol{d}_{2}\right)$ \\
3 & $\frac{a}{4}(-1,1,-1)$ & $e^{2 \pi i \frac{p}{q}\left(I^{\prime}+\frac{1}{4}\right)}$ & $e^{2 \pi i k_{2}} C_{\boldsymbol{k}}^{n l J M}\left(I^{\prime} \boldsymbol{a}_{1}+\boldsymbol{d}_{2}\right)$ \\
4 & $\frac{a}{4}(-1,-1,1)$ & $e^{2 \pi i \frac{p}{q}\left(I^{\prime}-\frac{1}{4}\right)}$ & $C_{\boldsymbol{k}}^{n l J M}\left(\left(I^{\prime}-1\right) \boldsymbol{a}_{1}+\boldsymbol{d}_{2}\right)$ \\
\hline \hline
\end{tabular}

Equation (92) includes an important suggestion for a way to determine the formula of the magnetic field. Here, we shall give an important comment on the formula of the magnetic field that is consistent with the magnetic Bloch theorem. If the magnetic field were given by

$$
B=\frac{8 \pi \hbar}{e a^{2}} \frac{p}{q}
$$

instead of Eq. (81), then the phase factor $e^{-i \frac{e B}{\hbar} T_{W x}\left(d_{j}\right) t_{m y}}$ that appears in Eq. (92) would not be equal to 1 and would depend on $t_{m y}$. In this case, the simultaneous equations for the set of coefficients $\left\{C_{\boldsymbol{k}}^{n l J M}\left(I_{\boldsymbol{a}_{1}}+\boldsymbol{d}_{i}\right) \mid I=0,1,2, \ldots, q-1 ; i=1,2 ; n l J M\right\}$ vary with $t_{m y}$ although the set of coefficients, of course intrinsically, does not depend on $t_{m y}$. This means that the original simultaneous equations [Eq. (60)] for the magnetic field (94) lead to solutions that are not consistent with the magnetic Bloch theorem [Eq. (91)]. This difficulty seems to come from the incompleteness of the set of basis functions $\left\{\psi_{\boldsymbol{R}_{n}+\boldsymbol{d}_{i}}^{\xi}(\boldsymbol{r})\right\}$. In order to avoid this difficulty, the magnitude of the magnetic field is chosen in the form of Eq. (81) [49]. Namely, if the form of the magnetic field is chosen like Eq. (81), then the phase factor $e^{-i \frac{e B}{\hbar} T_{W x}\left(\boldsymbol{d}_{j}\right) t_{m y}}$ is shown to be equal to 1 , which makes the solution of Eq. (92) consistent with the magnetic Bloch theorem. Considering this fact, i.e., $e^{-i \frac{e B}{\hbar} T_{W x}\left(\boldsymbol{d}_{j}\right) t_{m y}}=1$, Eq. (92) is finally rewritten as

$$
\begin{aligned}
& \left(\bar{\varepsilon}_{n^{\prime} l^{\prime} J^{\prime}}^{a_{i}}+\Delta \bar{\varepsilon}_{n^{\prime} l^{\prime} J^{\prime} M^{\prime}}^{a_{j}, \boldsymbol{d}_{j}}+\frac{e B}{2 m} \frac{2 J^{\prime}+1}{2 l^{\prime}+1} \hbar M^{\prime}\right) C_{\boldsymbol{k}}^{n^{\prime} l^{\prime} J^{\prime} M^{\prime}}\left(I^{\prime} \boldsymbol{a}_{1}+\boldsymbol{d}_{j}\right) \\
& \quad+\sum_{n l J M} \sum_{W} e^{-i \frac{e B}{2 \hbar} T_{W x}\left(\boldsymbol{d}_{j}\right)\left\{T_{W y}\left(\boldsymbol{d}_{j}\right)+2 I^{\prime} a_{1 y}+2 d_{j y}\right\}} t_{n^{\prime} l^{\prime} J^{\prime} M^{\prime}, n l J M}\left(\boldsymbol{T}_{W}\left(\boldsymbol{d}_{j}\right)\right) C_{\boldsymbol{k}}^{n l J M}\left(\boldsymbol{T}_{W}\left(\boldsymbol{d}_{j}\right)+I^{\prime} \boldsymbol{a}_{1}+\boldsymbol{d}_{j}\right)=E_{\boldsymbol{k}} C_{\boldsymbol{k}}^{n^{\prime} l^{\prime} J^{\prime} M^{\prime}}\left(I^{\prime} \boldsymbol{a}_{1}+\boldsymbol{d}_{j}\right) .
\end{aligned}
$$

\section{Concrete expressions for the simultaneous equations}

Let us give the concrete expressions for the simultaneous equations used in the actual calculations. In order to consider the electronic states in the vicinity of the Fermi level, we shall take $s$ and $p$ electrons of the outer shells of the Si atom in calculating the hopping integrals. Namely, we consider the hopping integrals between the following eight shells:

$$
(n, l, J, M)=\left(3,0, \frac{1}{2}, \pm \frac{1}{2}\right),\left(3,1, \frac{1}{2}, \pm \frac{1}{2}\right),\left(3,1, \frac{3}{2}, \pm \frac{1}{2}\right),\left(3,1, \frac{3}{2}, \pm \frac{3}{2}\right) .
$$

Furthermore, we consider only the electron hoppings between the nearest-neighbor atoms. Specifically, four kinds of vectors $\boldsymbol{T}_{W}\left(\boldsymbol{d}_{j}\right)(W=1-4)$ are considered in the calculations.

In the following, we show the concrete expression for Eq. (95) in the cases for (i) $\boldsymbol{d}_{j}=\boldsymbol{d}_{1}$ and (ii) $\boldsymbol{d}_{j}=\boldsymbol{d}_{2}$, individually. The phase factors $e^{-i \frac{e B}{2 \hbar} T_{W x}\left(\boldsymbol{d}_{j}\right)\left\{T_{W y}\left(\boldsymbol{d}_{j}\right)+2 I^{\prime} a_{1 y}+2 d_{j y}\right\}}$ and coefficients $C_{\boldsymbol{k}}^{n l J M}\left(\boldsymbol{T}_{W}\left(\boldsymbol{d}_{j}\right)+I^{\prime} \boldsymbol{a}_{1}+\boldsymbol{d}_{j}\right)$ in the left-hand side of Eq. (95) are given in Tables V and VI for both cases [(i) and (ii)]. Using these, we have

TABLE VI. Phase factors $e^{-i \frac{e B}{2 \hbar} T_{W x}\left(\boldsymbol{d}_{2}\right)\left(T_{W y}\left(\boldsymbol{d}_{2}\right)+2 I^{\prime} a_{1 y}+2 d_{2 y}\right)}$ and coefficients $C_{\boldsymbol{k}}^{n l J M}\left(\boldsymbol{T}_{W}\left(\boldsymbol{d}_{2}\right)+I^{\prime} \boldsymbol{a}_{1}+\boldsymbol{d}_{2}\right)$ that appear in the left-hand side of Eq. (95).

\begin{tabular}{cccc}
\hline \hline$W$ & $\boldsymbol{T}_{W}\left(\boldsymbol{d}_{2}\right)$ & $e^{-i \frac{e B}{2 \hbar} T_{W x}\left(\boldsymbol{d}_{2}\right)\left(T_{W y}\left(\boldsymbol{d}_{2}\right)+2 I^{\prime} a_{1 y}+2 d_{2 y}\right)}$ & $C_{\boldsymbol{k}}^{n l J M}\left(\boldsymbol{T}_{W}\left(\boldsymbol{d}_{2}\right)+I^{\prime} \boldsymbol{a}_{1}+\boldsymbol{d}_{2}\right)$ \\
\hline 1 & $\frac{a}{4}(1,1,1)$ & $e^{2 \pi i \frac{p}{q}\left(I^{\prime}+\frac{1}{2}\right)}$ & $C_{\boldsymbol{k}}^{n l J M}\left(I^{\prime} \boldsymbol{a}_{1}\right)$ \\
2 & $\frac{a}{4}(1,-1,-1)$ & $e^{2 \pi i \frac{p}{q}\left(I^{\prime}+\frac{3}{4}\right)}$ & $e^{-2 \pi i k_{3}} C_{\boldsymbol{k}}^{n l J M}\left(\left(I^{\prime}+1\right) \boldsymbol{a}_{1}\right)$ \\
3 & $\frac{a}{4}(-1,1,-1)$ & $e^{-2 \pi i \frac{p}{q}\left(I^{\prime}+\frac{1}{2}\right)}$ & $e^{-2 \pi i k_{2}} C_{\boldsymbol{k}}^{n l J M}\left(I^{\prime} \boldsymbol{a}_{1}\right)$ \\
4 & $\frac{a}{4}(-1,-1,1)$ & $e^{-2 \pi i \frac{p}{q}\left(I^{\prime}+\frac{3}{4}\right)}$ & $C_{\boldsymbol{k}}^{n l J M}\left(\left(I^{\prime}+1\right) \boldsymbol{a}_{1}\right)$ \\
\hline \hline
\end{tabular}


(i) $\boldsymbol{d}_{j}=\boldsymbol{d}_{1}$,

$$
\begin{aligned}
& \left(\bar{\varepsilon}_{3 l^{\prime} J^{\prime}} i \Delta \bar{\varepsilon}_{3 l^{\prime} J^{\prime} M^{\prime}}^{S i, \boldsymbol{d}_{1}}+\frac{e B}{2 m} \frac{2 J^{\prime}+1}{2 l^{\prime}+1} \hbar M^{\prime}\right) C_{\boldsymbol{k}}^{3 l^{\prime} J^{\prime} M^{\prime}}\left(I^{\prime} \boldsymbol{a}_{1}\right) \\
& \quad+\sum_{l J M}\left[e^{-2 \pi i \frac{p}{q}\left(I^{\prime}+\frac{1}{4}\right)} t_{3 l^{\prime} J^{\prime} M^{\prime}, 3 l J M}\left(\boldsymbol{T}_{1}\left(\boldsymbol{d}_{1}\right)\right)+e^{2 \pi i \frac{p}{q}\left(I^{\prime}+\frac{1}{4}\right)} e^{2 \pi i k_{2}} t_{3 l^{\prime} J^{\prime} M^{\prime}, 3 l J M}\left(\boldsymbol{T}_{3}\left(\boldsymbol{d}_{1}\right)\right)\right] C_{\boldsymbol{k}}^{3 l J M}\left(I^{\prime} \boldsymbol{a}_{1}+\boldsymbol{d}_{2}\right) \\
& \quad+\sum_{l J M}\left[e^{-2 \pi i \frac{p}{q}\left(I^{\prime}-\frac{1}{4}\right)} e^{2 \pi i k_{3}} t_{3 l^{\prime} J^{\prime} M^{\prime}, 3 l J M}\left(\boldsymbol{T}_{2}\left(\boldsymbol{d}_{1}\right)\right)+e^{2 \pi i \frac{p}{q}\left(I^{\prime}-\frac{1}{4}\right)} t_{3 l^{\prime} J^{\prime} M^{\prime}, 3 l J M}\left(\boldsymbol{T}_{4}\left(\boldsymbol{d}_{1}\right)\right)\right] C_{\boldsymbol{k}}^{3 l J M}\left(\left(I^{\prime}-1\right) \mathbf{a}_{1}+\boldsymbol{d}_{2}\right) \\
& =E_{\boldsymbol{k}} C_{\boldsymbol{k}}^{3 l^{\prime} J^{\prime} M^{\prime}}\left(I^{\prime} \boldsymbol{a}_{1}\right)
\end{aligned}
$$

with

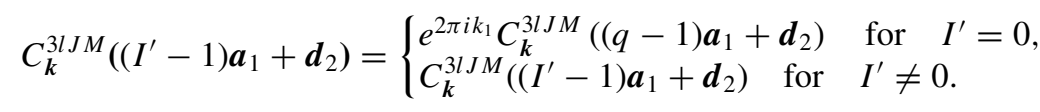

(ii) $\boldsymbol{d}_{j}=\boldsymbol{d}_{2}$,

$$
\begin{aligned}
\left(\bar{\varepsilon}_{3 l^{\prime} J^{\prime}}^{S i}+\Delta \bar{\varepsilon}_{3 l^{\prime} J^{\prime} M^{\prime}}^{S i, \boldsymbol{d}_{2}}+\frac{e B}{2 m} \frac{2 J^{\prime}+1}{2 l^{\prime}+1} \hbar M^{\prime}\right) C_{\boldsymbol{k}}^{3 l^{\prime} J^{\prime} M^{\prime}}\left(I^{\prime} \boldsymbol{a}_{1}+\boldsymbol{d}_{2}\right) \\
\quad+\sum_{l J M}\left[e^{2 \pi i \frac{p}{q}\left(I^{\prime}+\frac{1}{2}\right)} t_{3 l^{\prime} J^{\prime} M^{\prime}, 3 l J M}\left(\boldsymbol{T}_{1}\left(\boldsymbol{d}_{2}\right)\right)+e^{-2 \pi i \frac{p}{q}\left(I^{\prime}+\frac{1}{2}\right)} e^{-2 \pi i k_{2}} t_{3 l^{\prime} J^{\prime} M^{\prime}, 3 l J M}\left(\boldsymbol{T}_{3}\left(\boldsymbol{d}_{2}\right)\right)\right] C_{\boldsymbol{k}}^{3 l J M}\left(I^{\prime} \boldsymbol{a}_{1}\right) \\
\quad+\sum_{l J M}\left[e^{2 \pi i \frac{p}{q}\left(I^{\prime}+\frac{3}{4}\right)} e^{-2 \pi i k_{3}} t_{3 l^{\prime} J^{\prime} M^{\prime}, 3 l J M}\left(\boldsymbol{T}_{2}\left(\boldsymbol{d}_{2}\right)\right)+e^{-2 \pi i \frac{p}{q}\left(I^{\prime}+\frac{3}{4}\right)} t_{3 l^{\prime} J^{\prime} M^{\prime}, 3 l J M}\left(\boldsymbol{T}_{4}\left(\boldsymbol{d}_{2}\right)\right)\right] C_{\boldsymbol{k}}^{3 l J M}\left(\left(I^{\prime}+1\right) \boldsymbol{a}_{1}\right) \\
=E_{\boldsymbol{k}} C_{\boldsymbol{k}}^{3 l^{\prime} J^{\prime} M^{\prime}}\left(I^{\prime} \boldsymbol{a}_{1}+\boldsymbol{d}_{2}\right)
\end{aligned}
$$

with

$$
\begin{aligned}
& C_{k}^{3 l J M}\left(\left(I^{\prime}+1\right) \boldsymbol{a}_{1}\right) \\
& \quad=\left\{\begin{array}{lll}
e^{-2 \pi i k_{1}} C_{k}^{3 l J M}(0) & \text { for } & I^{\prime}=q-1, \\
C_{\boldsymbol{k}}^{3 l J M}\left(\left(I^{\prime}+1\right) \boldsymbol{a}_{1}\right) & \text { for } & I^{\prime} \neq q-1 .
\end{array}\right.
\end{aligned}
$$

In Eqs. (97) and (99), the summation on $l, J$, and $M$ is over eight states given in Eq. (96). The hopping integrals can be transcribed by the TB parameters with the aid of the relativistic Slater-Koster table shown in Table I. Equations (97) and (99) form simultaneous equations with a finite number of coefficients $\left\{C_{k}^{3 l J M}\left(I \boldsymbol{a}_{1}+\boldsymbol{d}_{i}\right) \mid I=0,1, \ldots, q-1 ; i=\right.$ $1,2 ;(3, l, J, M)=$ Eq. $(96)\}$.

\section{Energy bands for a crystalline silicon immersed in a magnetic field}

\section{Energy dispersion}

Figure 6 shows the energy bands for a crystalline silicon immersed in a uniform magnetic field, where $p$ and $q$ are fixed at 1 and 101, respectively. We have $16 q$ (=1616) energy bands in the energy dispersion because $16 q$ eigenvalues of $E_{\boldsymbol{k}}$ are obtained for each $\boldsymbol{k}$. The labels indicated in the horizontal axis of Fig. 6 denote the points in the magnetic first Brillouin zone [Fig. 5(c)]. Points $P, R_{x}$, and $A_{x}$ are quite close to each other owing to the present magnetic field. Their coordinates are explicitly given in Fig. 5(c). It is found from Fig. 6 that values of $E_{k}$ obviously change depending on $k_{z}(\Gamma-X-P$ line). This corresponds to the fact that the electron is not subjected to the Lorentz force in the $z$ direction, and makes the relatively large hopping along the $z$ direction. On the other hand, $E_{k}$ depends little on $k_{x}$ and $k_{y}$, and gap structures are observed in the $R_{x}-A_{x}-\Gamma$ line, similar to the case of the two-dimensional square lattice. Zooming in these flat bands for several cases [Figs. 7(a)-7(e)], we can find the following five properties in $E_{k}$ curves.

(i) It is found that $E_{k}$ is periodic in the $k_{x}-k_{y}$ plane. Similar to the case of the two-dimensional square lattice [50], this periodicity comes from the symmetry of the crystalline silicon immersed in the $z$-directed magnetic field. Namely, since $E_{k}$ is a periodic function of $k_{y}$ with the period of $4 \pi / a q$ that corresponds to the width of the magnetic first Brillouin zone, $E_{k}$ also becomes a periodic function of $k_{x}$ with the same period.

(ii) In order to investigate the gap structures observed in the $k_{x}-k_{y}$ plane, let us consider the case where the rational number $p / q$ in Eq. (81) is given by $1 / q(p=1)$. It is expected that the number of allowed bands is approximately proportional to $q$, because the number of energy bands $E_{k}$ is given by $16 q$. Indeed, we find a relation such that $N \propto 6 q$, where $N$ is the number of allowed bands, which is directly confirmed through numerical calculations. From this property, the bandwidth is expected to decrease with $q$. The magnetic-field dependence of the bandwidth will be discussed in property (iv).

(iii) Next, we consider the case where $p / q$ is nearly equal to $1 / q^{\prime}$, i.e., $q \approx p q^{\prime}$, where $q^{\prime}$ is a prime integer. Figures $7(\mathrm{a})-7$ (e) show energy bands in the $k_{x}-k_{y}$ plane for the cases of $1 / q^{\prime}=1 / 11, p / q=2 / 23,3 / 31,4 / 41$, and $5 / 53$, respectively. It is found from these figures that $p$ allowed bands are observed in the case of $p / q$, while one allowed band is observed in the case of $1 / q^{\prime}$. Therefore $N$ allowed bands for the case of $1 / q^{\prime}$ are respectively divided into $p$ allowed bands for the case of $p / q$. 


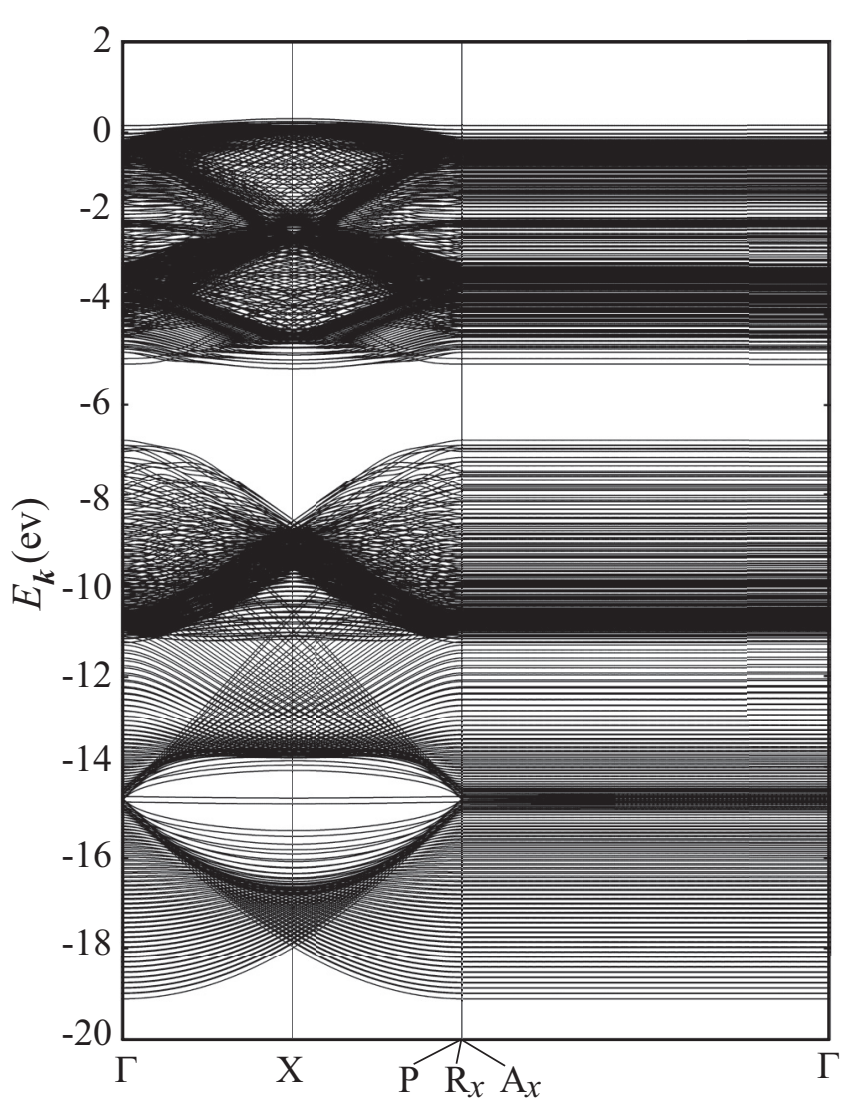

FIG. 6. Energy dispersions for a crystalline silicon immersed in a uniform magnetic field. The values of $p$ and $q$ are 1 and 101, respectively. The labels in the horizontal axis denote the special $\boldsymbol{k}$ points that are indicated in Fig. 5(c).

This property can be recognized as follows. Because of the relation $q \approx p q^{\prime}$, in the case of $p / q$, the period of $\boldsymbol{t}_{n}$ along the $\boldsymbol{a}_{1}$ direction is $p$ times longer than that in the case of $1 / q^{\prime}$. Therefore, due to the folding of the magnetic first Brillouin zone, $p$ energy gaps may be induced at the boundaries of the magnetic first Brillouin zone by the Bragg reflection. This is the reason why the number of allowed bands in the case of $p / q$ is $p$ times more than that in the case of $1 / q^{\prime}(\approx p / q)$.

(iv) In the case of $p / q$, the energy width of the cluster consisting of $p$ allowed bands is referred to as the cluster width. It is found from Figs. 7(a)-7(e) that the energy width of the allowed band for the case of $1 / q^{\prime}$ is nearly equal to the cluster width for the case of $p / q$. (Note that, although the cluster width in Fig. 7(b) is exceptionally a little smaller than those of other cases [Figs. 7(c)-7(e)], this discrepancy is not essential. This discrepancy comes from the error of the premise $1 / q^{\prime} \approx p / q$, i.e., the accuracy of the premise is not good in the case of $p / q=2 / 23$ as compared with other cases.)

Due to this property, we can say that the outlines of gap structures for five cases with $1 / q^{\prime} \approx p / q$ resemble each other if the full energy bands are plotted in the larger energy scale [Figs. 8(a)-8(e)]. Therefore we may regard the cluster width for the case of $p / q$ as the "bandwidth" that is nearly identical with the real bandwidth for the case of $1 / q^{\prime}$. (a) $p / q=1 / 11$

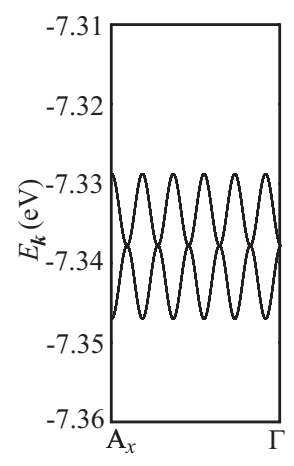

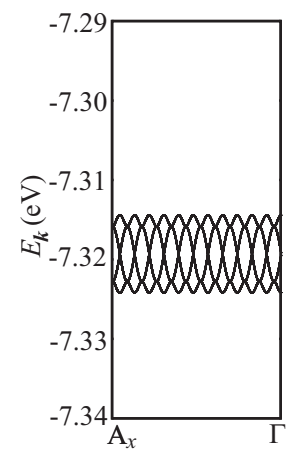

(b) $p / q=2 / 23$

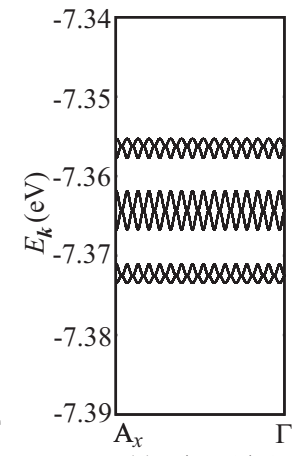

(c) $p / q=3 / 31$

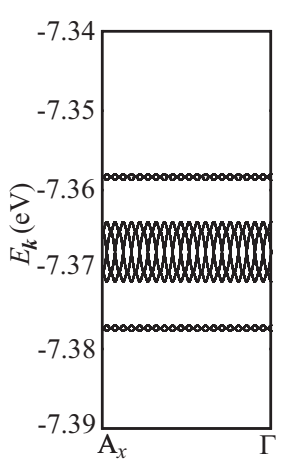

(d) $p / q=4 / 41$

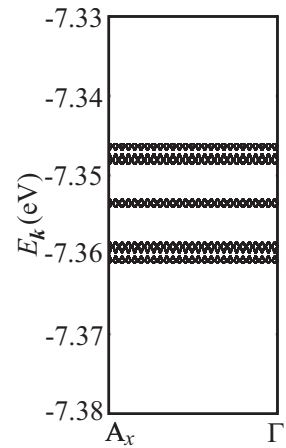

(e) $p / q=5 / 53$
FIG. 7. Magnified views of flat bands for several cases. Energy dispersions in the $k_{x}-k_{y}$ plane for the cases of (a) $p / q=1 / 11$, (b) $2 / 23$, (c) $3 / 31$, (d) $4 / 41$, and (e) $5 / 53$.

(v) As mentioned in property (iv), the gap structure for the case of $1 / q^{\prime}$ is similar to those for the cases of $p / q(\approx$ $\left.1 / q^{\prime}\right)$. So, we shall discuss the magnetic-field dependence of the bandwidth by using the bandwidth for the case of $1 / q^{\prime}$. Figures 9(a)-9(e) show the energy bands in the $k_{x}-k_{y}$ plane for the cases of moderately weak magnetic field $p / q<0.1$. It is found from these figures that there exist bandwidths that depend on the magnitude of the magnetic field. The bandwidths definitely increase as the magnetic field becomes large. When the magnetic field is quite weak, then the electronic states are comparably less affected by the magnetic field. At that time, the effective mass approximation seems to be valid, so that the property of the Landau level still survives in the electronic states. On the other hand, when the magnetic field increases, the electron hopping between the electronic states becomes larger, which results in making the bandwidth of the energy dispersion large.

This is easily comprehended from the following discussion. In the solids with no magnetic field, the electron hopping between the atomic orbitals generally takes some nonzero value, and correspondingly yields the nonzero width of the energy dispersion. As the magnetic field increases, the spatial broadening of the electronic state gets close to that of the usual atomic orbital, which may facilitate the electron hopping between two neighboring states. Therefore we can also say that the magnitude of the bandwidth corresponds to the degree of the infeasibility of the effective mass approximation in the region of moderately weak magnetic field $p / q<0.1$. 

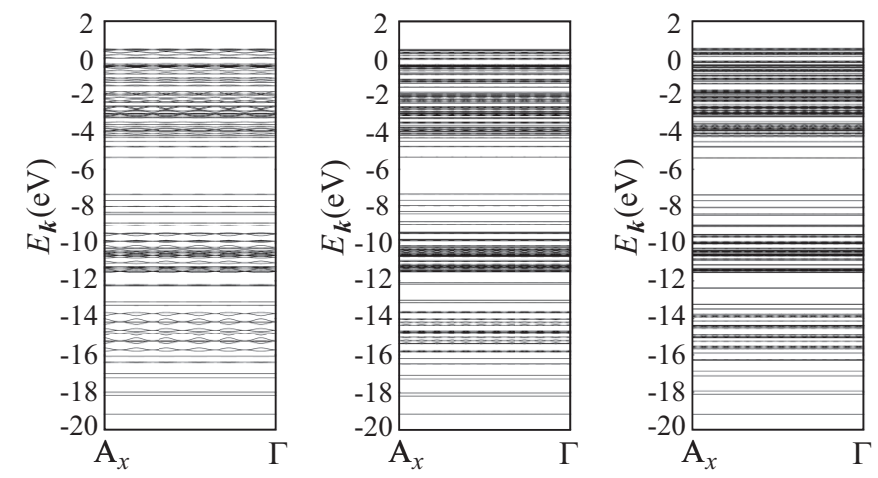

(a) $p / q=1 / 11$ (b) $p / q=2 / 23$

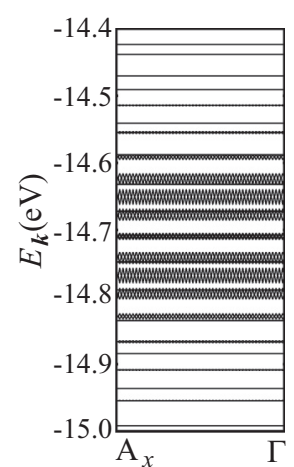

(a) $1 / q^{\prime}=1 / 101$

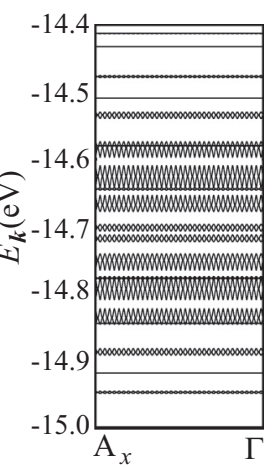

(b) $1 / q^{\prime}=1 / 71$

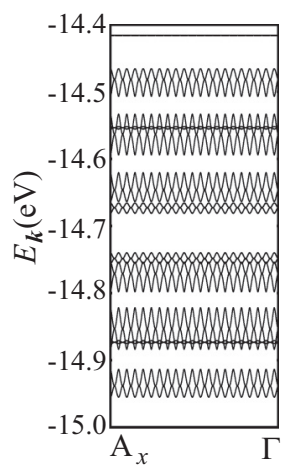

(c) $1 / q^{\prime}=1 / 41$

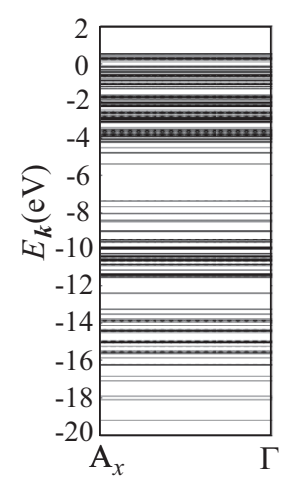

(d) $p / q=4 / 41$

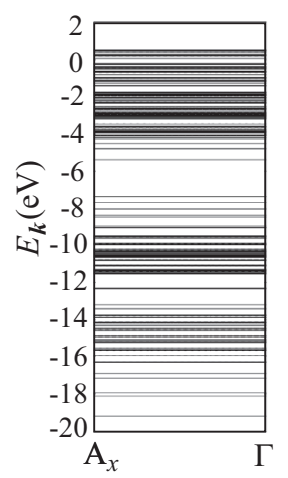

(e) $p / q=5 / 53$

FIG. 8. Whole energy dispersions in the $k_{x}-k_{y}$ plane for several cases. Energy dispersions in the $k_{x}-k_{y}$ plane for the cases of (a) $p / q=1 / 11$, (b) $2 / 23$, (c) $3 / 31$, (d) $4 / 41$, and (e) $5 / 53$.

\section{Butterfly diagram for a crystalline silicon immersed in a magnetic field}

The magnetic-field-dependent energy diagrams are shown in Figs. 10(a) and 10(b). These are calculated under two conditions. One is that all values of $E_{\boldsymbol{k}}$ for $\boldsymbol{k}$ 's that correspond to the horizontal axis of Fig. 5(c) are plotted in each magnetic field [Fig. 10(a)]. Another is that all values of $E_{\boldsymbol{k}}$ for $\boldsymbol{k}$ 's that are in the $k_{x}-k_{y}$ plane are plotted in each magnetic field [Fig. 10(b)]. Due to the Zeeman term, the energy diagrams of both figures become wide as the magnetic field increases. Correspondingly, the energy diagrams for the crystalline silicon are not symmetric, similar to the case of the twodimensional square lattice [Fig. 4(a)]. Comparing Fig. 10(a) with Fig. 10(b), the characteristic gap structures can be found in Fig. 10(b), while almost all of them disappear in Fig. 10(a). Especially, in Fig. 10(b), energy diagrams that are similar to the Hofstadter butterfly diagrams can be observed. In addition, recursive structures can be confirmed in Fig. 10(c). Since $q$ are prime numbers, and since a quite small range of $p / q$ is magnified in Fig. 10(c), the nonuniformity of plotted $p / q$, which inevitably emerges, is a little noticeable. However, we can definitely confirm the recursive patterns of energy gaps in Fig. 10(c).

It is easily understood that the disappearance of gap structures in Fig. 10(a) is due to the fact that values of $E_{\boldsymbol{k}}$ vary depending on $k_{z}$. However, the appearance of the butterfly
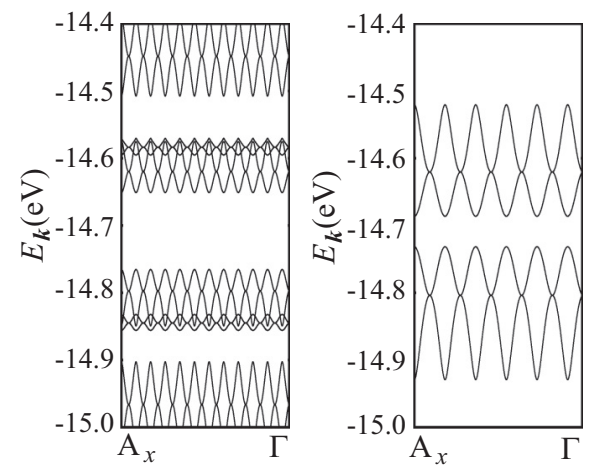

(d) $1 / q^{\prime}=1 / 23$

(e) $1 / q^{\prime}=1 / 11$

FIG. 9. Magnetic-field dependence of the energy dispersion for a crystalline silicon immersed in a uniform magnetic field. Energy dispersions in the $k_{x}-k_{y}$ plane for the cases of (a) $1 / q^{\prime}=1 / 101$, (b) $1 / 71$, (c) $1 / 41$, (d) $1 / 23$, and (e) $1 / 11$.

diagrams in Figs. 10(b) and 10(c) would indicate the peculiar properties of the electronic states of a crystalline silicon immersed in a magnetic field. The magnetic-field dependence of gap structures of $E_{\boldsymbol{k}}$ bands in the $k_{x}-k_{y}$ plane is essential for the appearance of the butterfly diagrams. This means that the electronic states of the crystalline silicon immersed in the magnetic field partially loose the two-dimensional degree of freedom depending on the magnitude of the magnetic field, though the crystalline silicon itself has three-dimensional structure. That is to say, the motion of electrons in the two-dimensional plane is restricted to a degree depending on the magnitude of the magnetic field, so that the energy diagram of the butterfly shape correspondingly emerges.

Thus it is shown by means of the MFRTB method that the butterfly diagram, which includes recursive structures, appears in the energy diagrams of a crystalline silicon immersed in a magnetic field. There is a future possibility that such energy structures are observed experimentally also in a silicon-based system as already observed in the GaAs/AlGaAs heterostructure system [51,52].

\section{Valence and conduction bands}

As can be seen in Figs. 10(a), 10(b), and 6, when the magnetic field is weak, an energy gap appears in the vicinity of $-6 \mathrm{eV}$. We shall focus on this energy gap, and show that it just corresponds to the energy gap between the valence 


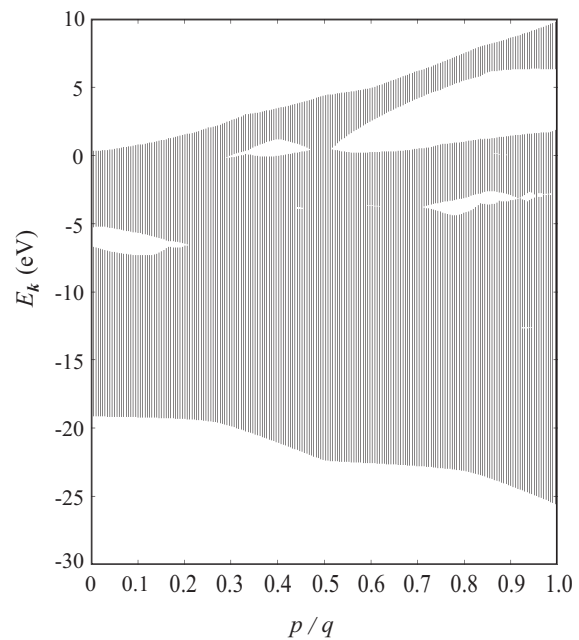

(a)

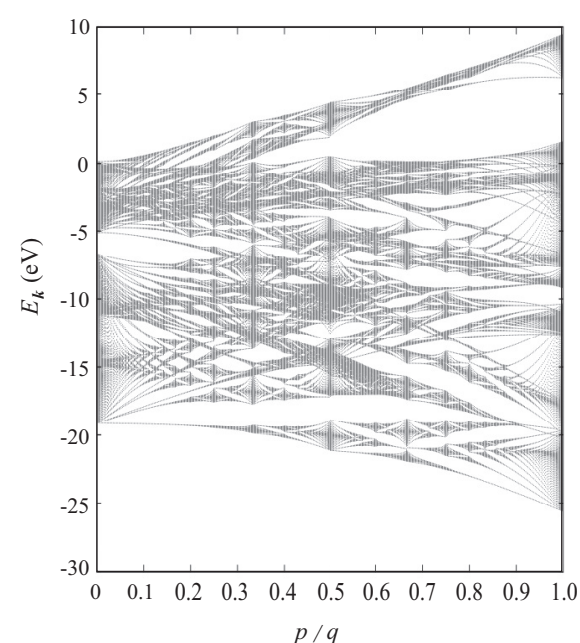

(b)

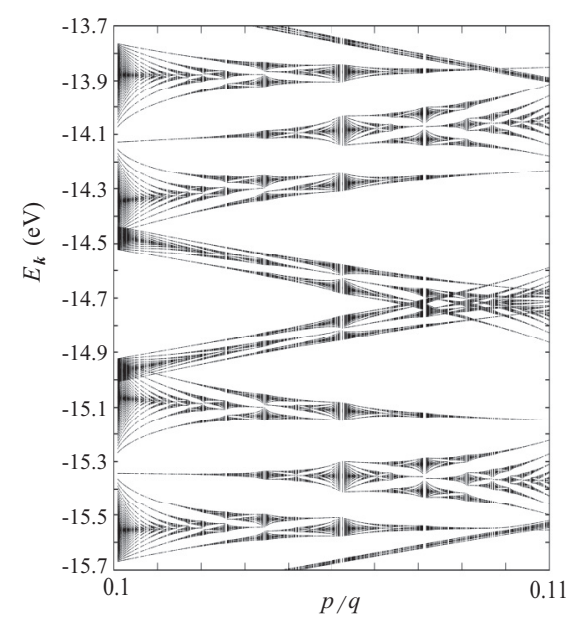

(c)

FIG. 10. Dependence of the energy diagram on the magnetic field for a crystalline silicon (a) in the case of varying wave vectors in the magnetic first Brillouin zone and (b) in the case of varying wave vectors in the $k_{x}-k_{y}$ plane of the magnetic first Brillouin zone. The magnified view of the energy diagram is shown in (c), where the recursive structures can be found.

and conduction bands. Although there exist $8 q$ energy bands bellow and above this energy gap, respectively, in Fig. 6, we will show that they correspond to the valence and conduction bands, respectively.

First, let us confirm the following two points. (i) As shown in Secs. IV B and V B, the magnetic Bloch theorem makes the diagonalization problem of deriving all the eigenvalues of the system split into that for deriving the eigenvalues of each $\boldsymbol{k}$ belonging to the magnetic first Brillouin zone. This statement is straightforwardly expressed by Eqs. (73) and (91). (ii) All electronic states of the system can be indicated by the wave numbers $\boldsymbol{k}$ that lie within the magnetic first Brillouin zone. This can be easily shown by using the magnetic Bloch theorem in a similar way to the case of no magnetic field [53]. Using these two points, it is proved that the number of $\boldsymbol{k}$ involved in the magnetic first Brillouin zone is equal to that of $\boldsymbol{t}_{n}$, which are distributed in the whole system. We shall denote such a number as $N_{t_{n}}$.

Next, we consider the number of electrons in the whole system. One lattice point has two Si atoms and correspondingly eight electrons (two sets of four outer-shell electrons) belong to it. As shown in Fig. 5(a), the "magnetic" primitive unit cell of the crystalline silicon immersed in the magnetic field has $q$ lattice points. Correspondingly, $8 q$ electrons are contained in the magnetic primitive unit cell. Since the system has $N_{t_{n}}$ points of $\boldsymbol{t}_{n}$, the number of electrons in the whole system is $8 q N_{t_{n}}$.

As mentioned above, the magnetic first Brillouin zone has $N_{\boldsymbol{t}_{n}}$ allowed points of $\boldsymbol{k}$, so that $8 q$ bands are occupied in order of energy. Namely, the $8 q$ bands bellow the energy gap that exists around $-6 \mathrm{eV}$ are valence bands, and the higher bands are conduction bands. Using this fact, it is also confirmed in Fig. 10(a) that the original energy gap of the crystalline silicon, which ranges from about $-6.5 \mathrm{eV}$ to $-5.3 \mathrm{eV}$ at $p / q=0$, remains up to the magnitude of the magnetic field, $p / q=0.2$. This would be due to both the increase of the top of the valence band and the decrease of the bottom of the conduction band that are mainly caused by the Zeeman term.

\section{CONCLUDING REMARKS}

We develop the MFRTB method that enables us to calculate the electronic structure of materials immersed in a uniform magnetic field. The formulation is accomplished in the orthodox coordinate representation. The striking features of the MFRTB method and findings obtained by this method are as follows.

(1) The MFRTB method enables us to calculate the electronic structure of actual crystalline materials immersed in a magnetic field by taking both relativistic and magnetic field effects into account.

(2) It is shown that within the lowest-order perturbation theory the magnetic hopping integrals are approximated as the relativistic hopping integrals multiplied by the Peierls phase factor. This approximation for the magnetic hopping integrals can be improved systematically by incorporating higher-order correction terms.

(3) The relativistic version of the Slater-Koster table is provided in Table I, in which relativistic hopping integrals are explicitly expressed in terms of relativistic TB parameters. Specifically, the explicit forms of 64 kinds of relativistic hopping integrals are given by the linear combination of relativistic TB parameters. The relativistic TB parameters can be readily obtained from electronic structure calculations for zero magnetic field case.

(4) An appropriate expression for the magnetic field, which leads to reasonable solutions consistent with the magnetic Bloch theorem, is revealed. In the case of the crystalline silicon, such a formula is given by Eq. (81).

(5) It is shown that the MFRTB method includes the Hofstadter's method [8]. Namely, if the MFRTB method is 
applied to the two-dimensional square lattice model with only $s$ electrons, and if the spin Zeeman term is neglected, then the MFRTB method reproduces the magnetic-field-dependent energy diagram, that is, the so-called Hofstadter butterfly diagram.

(6) We apply the MFRTB method to a crystalline silicon immersed in a uniform magnetic field. Recursive structures of energy spectra, i.e., butterfly patterns, can be seen in the $k_{x}-k_{y}$ plane of the magnetic first Brillouin zone [Figs. 10(b) and $10(\mathrm{c})$ ], but due to the $k_{z}$ dependence of energy bands, such characteristic structures disappear in the magnetic-fielddependent energy diagram [Fig. 10(a)]. Recursive structures are expected to be observed by experiments, e.g., angleresolved photoemission spectroscopy, and/or by preparing appropriate silicon-based systems such as the $\mathrm{Si} / \mathrm{SiC}$ heterostructure.

(7) It is also found that the widths of energy bands in the $k_{x}-k_{y}$ plane increase with the magnitude of the magnetic field [Figs. 9(a)-9(e)]. This suggests that the useful range of the effective mass approximation, which leads to the Landau levels, is limited to the region of the low magnetic field.
Thus we successfully obtain a first-principles method, i.e., MFRTB method, which can describe phenomena that are related to the magnetic Bloch electrons. Especially, the MFRTB method can be used to reveal the dependence of the elastic constants of a boron-doped silicon containing vacancies on the magnetic field [1]. The next work to be done is to consider the effects of boron dopants and vacancies contained in the crystalline silicon. To do so, we have to combine the present MFRTB method with the so-called supercell method [54], which is just our next task. Furthermore, using the MFRTB method, the alternative description of the dHvA effect from the view point of the first-principles calculation would be possible. This is also one of our future works [33]. In this manner, we expect that more evidence for the validity and usefulness of the MFRTB method will be accumulated through applications to various systems.

\section{ACKNOWLEDGMENTS}

This work was partially supported by Grant-in-Aid for Scientific Research (C) (No. 26400354 and No. 26400397) of Japan Society for the Promotion of Science.
[1] T. Goto, H. Yamada-Kaneta, Y. Saito, Y. Nemoto, K. Sato, K. Kakimoto, and S. Nakamura, J. Phys. Soc. Jpn. 75, 044602 (2006).

[2] H. Matsuura and K. Miyake, J. Phys. Soc. Jpn. 77, 043601 (2008).

[3] T. Yamada, Y. Yamakawa, and Y. Ono, J. Phys. Soc. Jpn. 78, 054702 (2009).

[4] S. Baba, T. Goto, Y. Nagai, M. Akatsu, H. Watanabe, K. Mitsumoto, T. Ogawa, Y. Nemoto, and H. Yamada-Kaneta, J. Phys. Soc. Jpn. 80, 094601 (2011).

[5] K. Okabe, M. Akatsu, S. Baba, K. Mitsumoto, Y. Nemoto, H. Yamada-Kaneta, T. Goto, H. Saito, K. Kashima, and Y. Saito, J. Phys. Soc. Jpn. 82, 124604 (2013).

[6] K. Mitsumoto, M. Akatsu, S. Baba, R. Takasu, Y. Nemoto, T. Goto, H. Yamada-Kaneta, Y. Furumura, H. Saito, K. Kashima, and Y. Saito, J. Phys. Soc. Jpn. 83, 034702 (2014).

[7] T. Ogawa, K. Tsuruta, and H. Iyetomi, Sol. State Comm. 151, 1605 (2011).

[8] D. R. Hofstadter, Phys. Rev. B. 14, 2239 (1976).

[9] M. P. Marder, Condensed Matter Physics (Wiley, New York, 2000), Chap. 25.

[10] Concerning the recent works, for instance, see the following works: P. Moon and M. Koshino, Phys. Rev. B 85, 195458 (2012); Y. Hatsugai, T. Fukui, and H. Aoki, ibid. 74, 205414 (2006); M. Koshino and H. Aoki, ibid. 67, 195336 (2003).

[11] Hofstadter's original method is based on the analytic form of the energy-dispersion relation $\left(\bar{E}_{\boldsymbol{k}}\right)$ for the case of zero magnetic field [8]. After deriving this analytic form, the Peierls replacement is applied to it to create the effective Hamiltonian for the system immersed in a magnetic field [8]. Such an effective Hamiltonian is diagonalized by using the atomic orbitals as basis functions. However, this calculation procedure is feasible only for a quite simplified system such as a single atom with only an $s$ electron located on each lattice point [8]. This is because the analytic form of $\bar{E}_{k}$ generally cannot be derived, if the order of the equation for $\bar{E}_{\boldsymbol{k}}$ is fifth or higher. This is known as the Abel-Ruffini theorem [12].

[12] For instance, see N. Jacobson, Basic Algebra I: Second Edition (Dover, New York, 2009), Chap. 4.

[13] The Peierls phase is used to construct the effective Hamiltonian for Bloch electrons in a magnetic field. Suppose that the TB Hamiltonian in the absence of the magnetic field is explicitly written in terms of the hopping integrals. By replacing the hopping integrals with those multiplied by the Peierls phase factor, the effective Hamiltonian in the presence of the magnetic field is given in this method [9].

[14] J. Zak, Phys. Rev. Lett. 19, 1385 (1967).

[15] J. Zak, Phys. Rev. 168, 686 (1968).

[16] J. Zak, Physics Today 23, 51 (1970).

[17] J. Zak, J. Phys. A: Math. Gen. 30, L549 (1997).

[18] G. M. Obermair and H.-J. Schellnhuber, Phys. Rev. B 23, 5185 (1981).

[19] H.-J. Schellnhuber, G. M. Obermair, and A. Rauh, Rhys. Rev. B 23, 5191 (1981).

[20] E. Anisimovas and P. Johansson, Phys. Rev. B 60, 7744 (1999).

[21] E. Anisimovas and P. Johansson, J. Phys.: Condens. Matter 13, 3365 (2001).

[22] J. Kübler, Theory of Itinerant Electron Magnetism (Oxford University Press, New York, 2000), Chap. 1.

[23] D. D. Koelling and G. O. Arbman, J. Phys. F 5, 2041 (1975).

[24] T. Takeda, Z. Phys. B 32, 43 (1978).

[25] M. Higuchi and A. Hasegawa, J. Phys. Soc. Jpn. 64, 830 (1995).

[26] M. R. Norman and D. D. Koelling, in Handbook on the Physics and Chemistry of Rare Earths, edited by K. A. Gschneidner Jr., L. Eyring, G. H. Lander, and G. R. Choppin (North-Holland, Amsterdam, 1993), Vol. 17, Chap. 110.

[27] Y. Onuki and A. Hasegawa, in Handbook on the Physics and Chemistry of Rare Earths, edited by K. A. Gschneidner Jr. 
and L. Eyring (North-Holland, Amsterdam, 1995), Vol. 20, Chap. 135.

[28] M. Higuchi and A. Hasegawa, J. Phys. Soc. Jpn. 65, 1302 (1996).

[29] Y. Tokiwa, T. Maehira, S. Ikeda, Y. Haga, E. Yamamoto, A. Nakamura, Y. Onuki, M. Higuchi, and A. Hasegawa, J. Phys. Soc. Jpn. 70, 2982 (2001).

[30] J. C. Slater and G. F. Koster, Phys. Rev. 94, 1498 (1954).

[31] K. Higuchi, K. Koide, T. Imanishi, and M. Higuchi, Int. J. Quantum Chem. 113, 709 (2013).

[32] P. G. de Gennes, Superconductivity of Metals and Alloys (Addison-Wesley, New York, 1966).

[33] D. B. Hamal, K. Higuchi, and M. Higuchi (unpublished).

[34] D. Shoenberg, Magnetic Oscillations in Metals (Cambridge University Press, Cambridge, 1984).

[35] A. Messiah, Quantum Mechanics (North-Holland, Amsterdam, 1966), Chap. 20.

[36] In general, the energy of the crystal field appears also in the off-diagonal elements of the Hamiltonian matrix. The general form of the effect of the crystal field is given by

$$
\begin{aligned}
& \int \varphi_{n^{\prime} l^{\prime} J^{\prime} M^{\prime}}^{a_{i}}\left(\boldsymbol{r}-\boldsymbol{d}_{i}\right)^{\dagger}\left\{\sum_{\boldsymbol{R}_{m}} \sum_{k} v_{a_{k}}\left(\boldsymbol{r}-\boldsymbol{R}_{m}-\boldsymbol{d}_{k}\right)\right\} \\
& \times\left(\boldsymbol{R}_{m}+\boldsymbol{d}_{k} \neq \boldsymbol{d}_{i}\right) \varphi_{n l J M}^{a_{i}}\left(\boldsymbol{r}-\boldsymbol{d}_{i}\right) d^{3} r .
\end{aligned}
$$

Since the relativistic atomic orbitals $\varphi_{n^{\prime} l^{\prime} J^{\prime} M^{\prime}}^{a_{i}}\left(\boldsymbol{r}-\boldsymbol{d}_{i}\right)$ and $\varphi_{n l J M}^{a_{i}}\left(\boldsymbol{r}-\boldsymbol{d}_{i}\right)$ are localized around $\boldsymbol{r}=\boldsymbol{d}_{i}$, we can reasonably approximate $v_{a_{k}}\left(\boldsymbol{r}-\boldsymbol{R}_{m}-\boldsymbol{d}_{k}\right)$ by the constant value at $\boldsymbol{r}=\boldsymbol{d}_{i}\left(v_{a_{k}}\left(\boldsymbol{d}_{i}-\boldsymbol{R}_{m}-\boldsymbol{d}_{k}\right)\right)$ in the above integrals. Due to the orthogonality of $\varphi_{n^{\prime} l^{\prime} J^{\prime} M^{\prime}}^{a_{i}}\left(\boldsymbol{r}-\boldsymbol{d}_{i}\right)$ and $\varphi_{n l J M}^{a_{i}}\left(\boldsymbol{r}-\boldsymbol{d}_{i}\right)$ for $\left(n^{\prime}, l^{\prime}, J^{\prime}, M^{\prime}\right) \neq(n, l, J, M)$, the above integral vanishes under this approximation. Therefore the off-diagonal elements of the energy of the crystal field are negligibly small as compared to the diagonal elements.

[37] R. M. Martin, Electronic Structure (Cambridge University Press, Cambridge, 2004), Chap. 14.

[38] P. Hohenberg and W. Kohn, Phys. Rev. 136, B864 (1964).

[39] W. Kohn and L. J. Sham, Phys. Rev. 140, A1133 (1965).

[40] Similar to the energy of the crystal field for the zero magnetic field case, the energy of the crystal field that appears in the offdiagonal elements can be neglected. For the nonzero magnetic field case, the off-diagonal elements of the energy of the crystal field is given by the following form:

$$
\begin{gathered}
\int \psi_{\eta}^{a_{i}, \boldsymbol{R}_{n}+\boldsymbol{d}_{i}}(\boldsymbol{r})^{\dagger}\left\{\sum_{\boldsymbol{R}_{m}} \sum_{k} v_{a_{k}}\left(\boldsymbol{r}-\boldsymbol{R}_{m}-\boldsymbol{d}_{k}\right)\right\} \\
\times\left(\boldsymbol{R}_{m}+\boldsymbol{d}_{k} \neq \boldsymbol{R}_{n}+\boldsymbol{d}_{i}\right) \psi_{\xi}^{a_{i}, \boldsymbol{R}_{n}+\boldsymbol{d}_{i}}(\boldsymbol{r}) d^{3} r,
\end{gathered}
$$

where $\eta \neq \xi$. The relativistic atomic orbital in the magnetic field is also localized in the vicinity of the atom as well as the relativistic atomic orbital of the zero magnetic field case. Therefore the approximation used in Ref. [32] is also applicable to the above integral. As a result, the orthogonality between $\psi_{\eta}^{a_{i}, \boldsymbol{R}_{n}+\boldsymbol{d}_{i}}(\boldsymbol{r})$ and $\psi_{\xi}^{a_{i}, \boldsymbol{R}_{n}+\boldsymbol{d}_{i}}(\boldsymbol{r})$ leads to an approximation such that the off-diagonal elements are negligible.

[41] We neglect the constant part of the solution in Eq. (38), because it gives only the trivial phase factor in Eq. (39), which is not expressed generally.

[42] Using Eq. (62), the magnitude of the magnetic field can be calculated by $B=1.403 \times 10^{4}(p / q) \mathrm{T}$ if we suppose $a=0.543 \mathrm{~nm}$, that is, the lattice constant of the silicon crystal.

[43] From Eq. (62), $p / q$ corresponds to the ratio of flux through a unit cell $\left(a^{2} B\right)$ to one flux quantum $(2 \pi \hbar / e)$. According to Hofstadter's paper [8], F. Bloch indicated that $p / q$ is recognized as the ratio of two kinds of periods: (1) the period of motion of an electron with the crystal momentum $2 \pi \hbar / a$, which is given by $a^{2} m / 2 \pi \hbar$, and (2) the reciprocal of the cyclotron frequency $e B / m$.

[44] For example, see E. Brown, Phys. Rev. 133, A1038 (1964).

[45] It is shown that $U\left(t_{n}\right)$ chosen like Eq. (67) takes the smallest period of the translation among the sets of $U\left(t_{n}\right)$ that form the Abelian group.

[46] The symmetry group of the Hamiltonian consists of the operators that commute with the Hamiltonian.

[47] T. Inui, Y. Tanabe, and Y. Onodera, Group Theory and Its Applications in Physics (Springer-Verlag, Berlin, 1990).

[48] Using Eq. (81), the magnitude of the magnetic field can be calculated by $B=1.122 \times 10^{5}(p / q)(\mathrm{T})$, where we use the lattice constant of silicon crystal $a=0.543(\mathrm{~nm})$.

[49] Also in the conventional TB method [37], there exists this kind of difficulty. In the conventional TB method [37], Bloch functions, which are constructed from the atomic orbitals and are called Bloch sum, are usually used as the basis functions, which makes the solution consistent with the Bloch theorem.

[50] Y. Hasegawa, Y. Hatsugai, M. Kohmoto, and G. Montambaux, Phys. Rev. B 41, 9174 (1990).

[51] C. Albrecht, J. H. Smet, K. von Klitzing, D. Weiss, V. Umansky, and H. Schweizer, Phys. Rev. Lett. 86, 147 (2001); Physica E (Amsterdam) 20, 143 (2003).

[52] M. C. Geisler, J. H. Smet, V. Umansky, K. von Klitzing, B. Naundorf, R. Ketzmerick, and H. Schweizer, Phys. Rev. Lett. 92, 256801 (2004)

[53] C. Kittel, Introduction to Solid State Physics (Wiley, New York, 2005), Chap. 9.

[54] For example, see, Sec. 13.4 of Ref. [37]. 